TRANSACTIONS OF THE

AMERICAN MATHEMATICAL SOCIETY

Volume 363, Number 11, November 2011, Pages 6007-6061

S 0002-9947(2011)05330-4

Article electronically published on June 15, 2011

\title{
CONSTRUCTIONS FOR INFINITESIMAL GROUP SCHEMES
}

\author{
ERIC M. FRIEDLANDER AND JULIA PEVTSOVA
}

\begin{abstract}
Let $G$ be an infinitesimal group scheme over a field $k$ of characteristic $p>0$. We introduce the global $p$-nilpotent operator $\Theta_{G}: k[G] \rightarrow k[V(G)]$, where $V(G)$ is the scheme which represents 1-parameter subgroups of $G$. This operator $\Theta_{G}$ applied to $M$ encodes the local Jordan type of $M$ and leads to computational insights into the representation theory of $G$. For certain $k G$ modules $M$ (including those of constant Jordan type), we employ $\Theta_{G}$ to associate various algebraic vector bundles on $\mathbb{P}(G)$, the projectivization of $V(G)$. These vector bundles not only distinguish certain representations with the same local Jordan type, but also provide a method of constructing algebraic vector bundles on $\mathbb{P}(G)$.
\end{abstract}

\section{Contents}

0. Introduction

1. Infinitesimal group schemes

2. Global $p$-nilpotent operators

3. Local Jordan type

4. $\pi$-points and $\mathbb{P}(\mathrm{G})$

5. Vector bundles for modules of constant $j$-rank $\quad 6039$

6. Examples and calculations with bundles $\quad 6051$

References

\section{INTRODUCTION}

In [25], 26], the foundations of a theory of support varieties were established for an infinitesimal group scheme $G$ over a field $k$ of characteristic $p>0$, extending earlier work for elementary abelian $p$-groups and $p$-restricted finite dimensional Lie algebras ([6], 13]). These foundations relied upon cohomological calculations to identify cohomological support varieties and introduced 1-parameter subgroups to provide an alternate, representation-theoretic perspective. In contrast to the situation for finite groups, the cohomological variety for $G$ infinitesimal is of considerable geometric complexity; partly for this reason, computations of explicit examples are

Received by the editors April 24, 2009 and, in revised form, February 9, 2010 and February $22,2010$.

2010 Mathematics Subject Classification. Primary 16G10, 20C20, 20G40.

The first author was partially supported by NSF grant \#0300525.

The second author was partially supported by NSF grant \#0629156.

(C)2011 American Mathematical Society Reverts to public domain 28 years from publication 
challenging. In this present paper, we build upon this earlier work as well as more recent work of the authors ([15], [17]) to initiate a more detailed investigation of representations of $G$. Although representations of infinitesimal group schemes are less familiar than representations of finite groups, their importance is evident: for example, the representation theories of the family of all infinitesimal kernels $G=\mathfrak{G}_{(r)}$ of a smooth connected algebraic group $\mathfrak{G}$ is essentially equivalent to the rational representation theory of $\mathfrak{G}$.

An important structure associated to an infinitesimal group scheme $G$ of height $\leq r$ is the scheme $V(G)$ of 1-parameter subgroups $\mathbb{G}_{a(r)} \rightarrow G$ (see [25]). In this paper we observe that the representability of $V(G)$ leads to a $p$-nilpotent element $\Theta_{G}$ in $k G \otimes k[V(G)]$, where $k G$ is the group algebra of $G$. For any $k G$-module $M$, $\Theta_{G}$ determines a global p-nilpotent operator on $M$. The operator $\Theta_{G}$ encodes the local Jordan type of a $k G$-module $M$ which in turn determines the support variety of $M$. Even though the scheme $V(G)$ was generalized to all finite group schemes in [15, [17] via the notion of $\pi$-points, the construction of $\Theta_{G}$ does not appear to extend to arbitrary finite groups.

The homogeneity of our global operator $\Theta_{G}$ enables us to associate to a $k G$ module $M$ of constant Jordan type a collection of vector bundles on $\mathbb{P}(G)=$ $\operatorname{Proj} k[V(G)]$, which we view as a family of global invariants of $M$. Certain modules with the same local Jordan type can be distinguished by these global invariants.

The vector bundles that we investigate are constructed directly and explicitly from $k G$-modules. Hence, we offer an important new method to create interesting examples of algebraic vector bundles on varieties of the form $\mathbb{P}(G)$. Varieties of this form include projective spaces, weighted projective spaces, and various singular varieties associated to algebraic groups. For example, for $G=\mathrm{GL}_{n(r)}, \mathbb{P}(G)$ is the projectivization of the variety of $r$-tuples of pairwise commuting $p$-nilpotent matrices. We expect that our technique of constructing algebraic vector bundles on such singular, geometrically interesting varieties will lead to insights into their algebraic K-theory.

The reader might find it instructive to contrast our use of representations of $G$ to construct vector bundles on $\mathbb{P}(G)$ with the Borel-Weil construction which employs bundles on flag varieties for an algebraic group $\mathfrak{G}$ to construct representations of $\mathfrak{G}$. Our construction of vector bundles plays a role in the forthcoming papers by the first author, Jon Carlson, and Andrei Suslin [9], and by the second author and David Benson [5].

In this paper, we also attempt to address the lack of specific examples in the representation theory of infinitesimal groups schemes (other than those of height 1). Throughout this paper, we work with the following four fundamental, yet concrete, classes of examples.

i) $p$-restricted Lie algebras, $\mathfrak{g}$;

ii) infinitesimal additive group schemes, $\mathbb{G}_{a(r)}$;

iii) infinitesimal general linear groups, $\mathrm{GL}_{n(r)}$;

iv) the height 2 , infinitesimal special linear group, $\mathrm{SL}_{2(2)}$.

We consistently endeavor to make our general results more concrete by applying them to our examples.

In Section 1, we recall some of the highlights from [25], [26] concerning the cohomology and theory of supports of finite dimensional $k G$-modules for an infinitesimal group scheme $G$. A key result summarized in Theorem 1.16 is the close relationship 
between the spectrum Spec $\mathrm{H}^{\bullet}(G, k)$ of the cohomology of $G$ and the scheme $V(G)$ representing (infinitesimal) 1-parameter subgroups of an infinitesimal group scheme $G$.

In the second section, we define the global p-nilpotent operator $\Theta_{G}: k[G] \longrightarrow$ $k[V(G)]$ for an infinitesimal group scheme $G$. For any finite dimensional $k G$-module $M, \Theta_{G}$ determines a $p$-nilpotent endomorphism of the free $k[V(G)]$-module $M \otimes$ $k[V(G)]$. We establish in Proposition 2.11 that $\Theta_{G}$ is homogeneous, where $k[V(G)]$ is equipped with its natural grading. We also verify that $\Theta_{G}$ is natural with respect to change of group.

In the third section, we verify that specializations $\theta_{v}$ of $\Theta_{G}$ at points $v \in V(G)$ determine the local Jordan type of a finite dimensional $k G$-module $M$. Theorem 3.7 can be viewed as providing an algorithm for obtaining the local Jordan type in terms of the representation $G \rightarrow \mathrm{GL}_{N}$ defining the $k G$-module $M$. We utilize $\Theta_{G}$ and its specializations to establish constraints for a $k G$-module $M$ to be of constant rank (and thus of constant Jordan type). We also establish the relationship between the local Jordan type of a module and its Frobenius twists.

We envision that some of our constructions for infinitesimal group schemes may lead to analogues for a general finite group scheme. With this in mind, we begin the fourth section with a dictionary between 1-parameter subgroups for infinitesimal group schemes and $\pi$-points for general finite group schemes. Given a finite dimensional $k G$-module $M$, we consider the projectivization of the operator $\Theta_{G}$,

$$
\widetilde{\Theta}_{G}: M \otimes \mathcal{O}_{\mathbb{P}(\mathrm{G})} \rightarrow M \otimes \mathcal{O}_{\mathbb{P}(\mathrm{G})}\left(p^{r-1}\right),
$$

a $p$-nilpotent operator on the free, coherent sheaf $M \otimes \mathcal{O}_{\mathbb{P}(\mathrm{G})}$ on $\mathbb{P}(\mathrm{G})$. We verify in Proposition 4.8 that $\widetilde{\Theta}_{G}$ determines via base change the local Jordan type of a $k G$-module $M$ at any 1-parameter subgroup $\mu_{v}: \mathbb{G}_{a(r), k(v)} \rightarrow G_{k(v)}$. Theorem 4.13 shows that the condition that $M$ be of constant $j$-rank is equivalent to the condition that the coherent sheaf $\operatorname{Im} \widetilde{\Theta}_{G}^{j}$ be locally free.

In the fifth section, we initiate an investigation of the algebraic vector bundles $\operatorname{Ker}\left\{\widetilde{\Theta}_{G}^{j}, \mathcal{M}\right\}, \operatorname{Im}\left\{\widetilde{\Theta}_{G}^{j}, \mathcal{M}\right\}$ on $\mathbb{P}(G)$ associated to $k G$-modules of constant Jordan type and more generally of constant $j$-rank. We give examples of such $k G$-modules in each of our four representative examples and investigate the associated vector bundles. As we see, taking kernels of powers of the global $p$-nilpotent power operator sends modules of constant Jordan type to vector bundles. We also obtain vector bundles by taking kernels modulo images (as inspired by a construction of M. Duflo and V. Serganova for Lie superalgebras in [11]). As an application, we prove in Proposition 5.17 a geometric characterization of endotrivial modules.

Finally, in the last section, we provide numerous explicit examples. These include the infinitesimal group scheme $G=\mathbb{G}_{a(1)} \times \mathbb{G}_{a(1)}$, which has the same representation theory as the elementary abelian $p$-group $\mathbb{Z} / p \times \mathbb{Z} / p$, as well as the first Frobenius kernel of the reductive group $\mathrm{SL}_{2}$. One intriguing comparison which we investigate in particularly simple examples is the relationship between the Grothendieck group of projective $k G$-modules and the Grothendieck group of algebraic vector bundles on $\mathbb{P}(G)$. Combined with our explicit calculations, Proposition 6.12 can be viewed both as a means to distinguish certain non-isomorphic projective $k G$-modules and as a means of constructing non-isomorphic algebraic vector bundles on $\mathbb{P}(\mathrm{G})$.

Throughout, $k$ will denote an arbitrary field of characteristic $p>0$. Unless explicit mention is made to the contrary, $G$ will denote an infinitesimal group 
scheme over $k$. If $M$ is a $k G$-module and $K / k$ is a field extension, then we denote by $M_{K}$ the $K G$-module obtained by base extension.

The authors are grateful to the University of Bielefeld and to MSRI for their hospitality. We thank Dan Grayson for pointing out the occurrence of weighted projective spaces and Paul Smith for numerous useful conversations.

\section{INFINITESIMAL GROUP SCHEMES}

The purpose of this first section is to summarize the important role played by (infinitesimal) 1-parameter subgroups of an infinitesimal group scheme as presented in 25. The four representative examples of Example 1.5. ( $\left.\mathfrak{g}, \mathbb{G}_{a(r)}, \mathrm{GL}_{n(r)}, \mathrm{SL}_{2(2)}\right)$, and their associated schemes of 1-parameter subgroups discussed in Example 1.12 will serve as explicit models to which we will frequently return.

Definition 1.1. A finite group scheme $G$ over $k$ is a group scheme over $k$ whose coordinate algebra $k[G]$ is finite dimensional over $k$.

Equivalently, $G$ is a functor from commutative $k$-algebras to groups, $R \mapsto G(R)$, represented by a finite dimensional commutative $k$-algebra, the coordinate algebra $k[G]$ of $G$.

Associated to $G$, we have its group algebra $k G=\operatorname{Hom}_{k}(k[G], k)$; more generally, for any commutative $k$-algebra $R$, we have the $R$-group algebra $R G=$ $\operatorname{Hom}_{k}(k[G], R)$.

Notation 1.2. If $f: G \rightarrow H$ is a map of finite group schemes, we denote by

$$
f^{*}: k[H] \rightarrow k[G] \quad \text { and } \quad f_{*}: k G \rightarrow k H
$$

the induced maps on coordinate and group algebras respectively.

Observe that the $R$-group algebra of $G$ consists of all $k$-linear homomorphisms, whereas $G(R)=\operatorname{Hom}_{k-a l g}(k[G], R)$ is the subgroup of $R G^{\times}$consisting of $k$-algebra homomorphisms.

Definition 1.3. Let $G$ be a finite group scheme over $k$ and $M$ a $k$-vector space. Then a (left) $k G$-module structure on $M$ is given by one of the following equivalent sets of data (see, for example, [22]):

- The structure $M \rightarrow M \otimes k[G]$ of a (right) $k[G]$-comodule on $M$.

- The structure $k G \otimes M \rightarrow M$ of a $k G$-module on $M$.

- A functorial (with respect to $R$ ) group action $G(R) \times(R \otimes M) \rightarrow(R \otimes M)$.

For most of this paper we shall restrict our consideration to infinitesimal group schemes, a special class of finite group schemes which we now define.

Definition 1.4. An infinitesimal group scheme $G$ (over $k$ ) of height $\leq r$ is a finite group scheme whose coordinate algebra $k[G]$ is a local algebra with maximal ideal $\mathfrak{m}$ such that $x^{p^{r}}=0$ for all $x \in \mathfrak{m}$.

Example 1.5. We shall frequently consider the following four examples.

(1) A finite dimensional $p$-restricted Lie algebra $\mathfrak{g}$ corresponds naturally with a height 1 infinitesimal group scheme which we denote $\mathfrak{g}$ ([22, I.8.5]). The group algebra of $\mathfrak{g}$ is the restricted enveloping algebra $\mathfrak{u}(\mathfrak{g})$ of $\mathfrak{g}$. If $\mathfrak{g}$ is the Lie algebra of a group scheme $\mathfrak{G}$, then the coordinate algebra of $\underline{\mathfrak{g}}$ is given by $k[\mathfrak{G}] /\left(x^{p}, x \in \mathfrak{m}\right)$, where $\mathfrak{m}$ is the maximal ideal of $k[\mathfrak{G}]$ at the identity of $\mathfrak{G}$. 
(2) Let $\mathbb{G}_{a}$ denote the additive group, so that $k\left[\mathbb{G}_{a}\right]=k[T]$ with coproduct defined by $\nabla(T)=T \otimes 1+1 \otimes T$. As a functor, $\mathbb{G}_{a}:(\mathrm{comm} k-$ alg $) \rightarrow($ grps $)$ sends an algebra $R$ to its underlying abelian group. For any $r \geq 1$, we consider the $r^{\text {th }}$ Frobenius kernel of $\mathbb{G}_{a}$,

$$
\mathbb{G}_{a(r)} \equiv \operatorname{Ker}\left\{F^{r}: \mathbb{G}_{a} \rightarrow \mathbb{G}_{a}\right\} .
$$

Here $F: \mathbb{G}_{a} \rightarrow \mathbb{G}_{a}$ is the (geometric) Frobenius specified by its map on coordinate algebras $k[T] \rightarrow k[T]$ given as the $k$-linear map sending $T$ to $T^{p}$. The coordinate algebra of $\mathbb{G}_{a(r)}$ is given by $k\left[\mathbb{G}_{a(r)}\right]=k[T] / T^{p^{r}}$, whereas the group algebra of $\mathbb{G}_{a(r)}$ is given by

$$
k \mathbb{G}_{a(r)} \simeq k\left[\mathbb{G}_{a(r)}\right]^{\#} \simeq k\left[u_{0}, \ldots, u_{r-1}\right] /\left(u_{0}^{p}, \ldots, u_{r-1}^{p}\right),
$$

where $u_{i}$ is a linear dual to $T^{p^{i}}, 0 \leq i \leq r-1$.

(3) Let $\mathrm{GL}_{n}$ denote the general linear group, the representable functor sending a commutative algebra $R$ to the group $\operatorname{GL}_{n}(R)$. For any $r \geq 1$, we consider the $r^{\text {th }}$ Frobenius kernel of $\mathrm{GL}_{n}$,

$$
\mathrm{GL}_{n(r)} \equiv \operatorname{Ker}\left\{F^{r}: \mathrm{GL}_{n} \rightarrow \mathrm{GL}_{n}\right\},
$$

where the geometric Frobenius

$$
F: \mathrm{GL}_{n}(R) \rightarrow \mathrm{GL}_{n}(R)
$$

is defined by raising each matrix entry to the $p^{\text {th }}$ power. The coordinate algebra of $\mathrm{GL}_{n(r)}$ is given by

$$
k\left[\mathrm{GL}_{n(r)}\right]=\frac{k\left[X_{i, j}\right]}{\left(X_{i, j}^{p^{r}}-\delta_{i, j}\right)}{ }_{1 \leq i, j \leq n},
$$

whereas the group algebra of $\mathrm{GL}_{n(r)}$ is given as

$$
k \mathrm{GL}_{n(r)}=\operatorname{Hom}_{k}\left(k\left[\mathrm{GL}_{n(r)}\right], k\right)
$$

the $k$-space of linear functionals $k\left[\mathrm{GL}_{n(r)}\right]$ to $k$. The coproduct

$$
\nabla: k\left[\mathrm{GL}_{n(r)}\right] \rightarrow k\left[\mathrm{GL}_{n(r)}\right] \otimes k\left[\mathrm{GL}_{n(r)}\right]
$$

is given by sending $X_{i, j}$ to $\sum_{k} X_{i k} \otimes X_{k j}$.

(4) The height 2 infinitesimal group scheme $\mathrm{SL}_{2(2)}$ is essentially a special case of $\mathrm{GL}_{n(r)}$. This is once again defined as the kernel of the second iterate of Frobenius,

$$
\mathrm{SL}_{2(2)} \equiv \operatorname{Ker}\left\{F^{2}: \mathrm{SL}_{2} \rightarrow \mathrm{SL}_{2}\right\} \text {. }
$$

The coordinate algebra of $\mathrm{SL}_{2(2)}$ is given by

$$
k\left[\mathrm{SL}_{2(2)}\right]=\frac{k\left[X_{1,1}, X_{1,2}, X_{2,1}, X_{2,2}\right]}{\left(X_{1,1} X_{2,2}-X_{1,2} X_{2,1}-1, X_{i, j}^{p^{2}}-\delta_{i, j}\right)},
$$

whereas the group algebra of $\mathrm{SL}_{2(2)}$ is given as

$$
k \mathrm{SL}_{2(2)}=k\left\langle e, f, h, e^{(p)}, f^{(p)}, h^{(p)}\right\rangle /\langle\text { relations }\rangle
$$

with $e, f, h, e^{(p)}, f^{(p)}, h^{(p)}$ the dual basis vectors to $X_{1,2}, X_{2,1}, X_{1,1}-1, X_{1,2}^{p}$, $X_{2,1}^{p},\left(X_{1,1}-1\right)^{p}$ respectively.

We denote by $\mathbb{G}_{a(r), R}$ the base extension of $\mathbb{G}_{a(r)}$ to a commutative $k$-algebra $R$.

Definition 1.6. An (infinitesimal) 1-parameter subgroup of height $r$ of an affine group scheme $G_{R}$ over a commutative $k$-algebra $R$ is a homomorphism of $R$-group schemes $\mathbb{G}_{a(r), R} \rightarrow G_{R}$. 
We recall the description of height $r$ 1-parameter subgroups of $\mathrm{GL}_{n}$ given in [25].

Proposition 1.7 ([25, 1.2]). If $G=\mathrm{GL}_{n}$ and if $R$ is a commutative $k$-algebra, then a 1-parameter subgroup of $\mathrm{GL}_{n, R}$ of height $r, f: \mathbb{G}_{a(r), R} \rightarrow \mathrm{GL}_{n, R}$, is naturally (with respect to $R$ ) equivalent to a comodule map

$$
\Delta_{f}: R^{n} \rightarrow R[T] / T^{p^{r}} \otimes_{R} R^{n}, \quad \Delta_{f}(v)=\sum_{j=0}^{p^{r-1}} T^{j} \otimes \beta_{j}(v), \quad \beta_{j} \in M_{n}(R)
$$

satisfying the constraints of being counital and coassociative. This in turn is equivalent to specifying an $r$-tuple of matrices $\alpha_{0}=\beta_{0}, \alpha_{1}=\beta_{p}, \ldots, \alpha_{r-1}=\beta_{p^{r-1}}$ in $M_{n}(R)$ such that each $\alpha_{i}$ has $p^{\text {th }}$ power 0 and such that the $\alpha_{i}$ 's pairwise commute. The other coefficient matrices $\beta_{j}$ are given by the formula

$$
\beta_{j}=\frac{\alpha_{0}^{j_{0}} \cdots \alpha_{r-1}^{j_{r-1}}}{\left(j_{0}\right) ! \cdots\left(j_{r-1}\right) !} \in M_{n}(R), \quad j=\sum_{i=0}^{r-1} j_{i} p^{i} \text { with } 0 \leq j_{i}<p .
$$

As shown in 25, Proposition 1.7 implies the following representability of the functor of 1-parameter subgroups of height $r$.

Theorem 1.8 ([25, 1.5]). For any affine group scheme $G$, the functor from commutative $k$-algebras to sets

$$
R \mapsto \operatorname{Hom}_{\text {grp sch }}\left(\mathbb{G}_{a(r), R}, G_{R}\right)
$$

is representable by an affine scheme $V_{r}(G)=\operatorname{Spec} k\left[V_{r}(G)\right]$. Namely, this functor is naturally isomorphic to the functor

$$
R \mapsto \operatorname{Hom}_{\mathrm{k}-\operatorname{alg}}\left(k\left[V_{r}(G)\right], R\right) .
$$

By varying $r$, we can associate a family of affine schemes to an affine group scheme $G$. In the following remark we make explicit the relationship between various $V_{r}(G)$ for the same $G$ and varying $r$ 's.

Remark 1.9. For $r>s \geq 1$, let $p_{r, s}: \mathbb{G}_{a(r)} \rightarrow \mathbb{G}_{a(s)}$ be the canonical projection given by the natural embedding of the coordinate algebras

$$
p_{r, s}^{*}: k\left[\mathbb{G}_{a(s)}\right]=k[T] / T^{p^{s}} \stackrel{T \rightarrow T^{p^{r-s}}}{\longrightarrow} k[T] / T^{p^{r}}=k\left[\mathbb{G}_{a(r)}\right] .
$$

The corresponding map on group algebras,

$$
k \mathbb{G}_{a(r)} \simeq k\left[u_{0}, \ldots, u_{r-1}\right] /\left(u_{0}^{p}, \ldots, u_{r-1}^{p}\right) \stackrel{p_{r, s, *}}{\longrightarrow} k \mathbb{G}_{a(s)} \simeq k\left[v_{0}, \ldots, v_{s-1}\right] /\left(v_{0}^{p}, \ldots, v_{s-1}^{p}\right),
$$

sends $\left\{u_{0}, \ldots, u_{r-s-1}\right\}$ to $\{0, \ldots, 0\}$ and $\left\{u_{r-s}, \ldots, u_{r-1}\right\}$ to $\left\{v_{0}, \ldots, v_{s-1}\right\}$.

Precomposition with $p_{r, s}$ determines a canonical embedding of affine schemes,

$$
i_{s, r}: V_{s}(G) \longleftrightarrow V_{r}(G),
$$

where a 1-parameter subgroup $\mu: \mathbb{G}_{a(s), R} \rightarrow G_{R}$ of height $s$ is sent to the 1parameter subgroup $\mu \circ p_{r, s}: \mathbb{G}_{a(r), R} \rightarrow \mathbb{G}_{a(s), R} \rightarrow G_{R}$ of height $r$. The construction is transitive, that is, we have $i_{s, r}=i_{s^{\prime}, r} \circ i_{s, s^{\prime}}$ for $s \leq s^{\prime} \leq r$. Hence, we have an inductive system

$$
V_{1}(G) \subset V_{2}(G) \subset \cdots \subset V_{r}(G) \subset \cdots .
$$


Conversely, any 1-parameter subgroup $\mathbb{G}_{a\left(s^{\prime}\right), R} \rightarrow G_{R}$ can be decomposed as

$$
\mathbb{G}_{a\left(s^{\prime}\right), R} \stackrel{p_{s^{\prime}, s}}{\longrightarrow} \mathbb{G}_{a(s), R} \stackrel{\longrightarrow}{\longrightarrow} G_{R}
$$

for some $s \leq s^{\prime}$. If $G$ is an infinitesimal group scheme of height $\leq r$, then we may choose $s \leq r$. This justifies the following definition.

Definition 1.10. Let $G$ be an infinitesimal group scheme. Then the closed immersion $i_{r, r^{\prime}}: V_{r}(G) \hookrightarrow V_{r^{\prime}}(G)$ for $r^{\prime}>r$ is an isomorphism provided the height of $G$ is $\leq r$. We denote by $V(G)$ the stable value of $V_{r}(G)$,

$$
V(G) \equiv \underset{r}{\longrightarrow} V_{r}(G) \text {. }
$$

We next make explicit the construction of 1-parameter subgroups for $\mathrm{GL}_{n}$ as in Proposition 1.7. This construction can be applied to any affine group scheme of exponential type (see [25, §1] and also [23] for an extended list of groups of exponential type). We define the homomorphism

$$
\exp _{\underline{\alpha}}: \mathbb{G}_{a(r), R} \rightarrow \mathrm{GL}_{n, R}
$$

of $R$-group schemes corresponding to an $r$-tuple $\underline{\alpha}=\left(\alpha_{0}, \ldots, \alpha_{r-1}\right) \in M_{n}(R)^{\times r}$ of pairwise commuting $p$-nilpotent matrices to be the natural transformation of groupvalued functors on commutative $R$-algebras $S$ sending any $s \in S$ with $s^{p^{r}}=0$ to

$$
\exp \left(s \alpha_{0}\right) \cdot \exp \left(s^{p} \alpha_{1}\right) \cdots \exp \left(s^{p^{r-1}} \alpha_{r-1}\right) \in \mathrm{GL}_{n}(S)
$$

where for any $p$-nilpotent matrix $A \in \mathrm{GL}_{n}(S)$ we set

$$
\exp (A)=1+A+\frac{A^{2}}{2}+\cdots+\frac{A^{p-1}}{(p-1) !} .
$$

The following proposition proved in 25] identifies the functor of 1-parameter subgroups in the case of infinitesimal general linear groups.

Proposition 1.11 ([25, 1.2]). The scheme of 1-parameter subgroups $V_{r}\left(\mathrm{GL}_{n}\right)$ is isomorphic to the scheme of $r$-tuples of pairwise commuting $p$-nilpotent $n \times n$ matrices $N_{p}^{[r]}\left(g l_{n}\right)$. The identification is given by sending $\underline{\alpha}=\left(\alpha_{0}, \ldots, \alpha_{r-1}\right) \in$ $N_{p}^{[r]}\left(g l_{n}\right)(R)$ to the 1-parameter subgroup $\exp _{\underline{\alpha}}: \mathbb{G}_{a(r), R} \rightarrow \mathrm{GL}_{n, R}$.

Example 1.12. We describe $V(G)$ in each of the four examples of Example 1.5.

(1) $V(\mathfrak{g}) \simeq N_{p}(\mathfrak{g})$, the closed subvariety of the affine space underlying $\mathfrak{g}$ consisting of $p$-nilpotent elements $x \in \mathfrak{g}$ (that is, $x^{[p]}=0$ ). Let $\mathfrak{g}_{a}$ be the Lie algebra of the additive group $\mathbb{G}_{a}$. Note that $\mathfrak{g}_{a}$ is a one dimensional restricted Lie algebra with trivial $p$-restriction. Each $p$-nilpotent element $x \in \mathfrak{g}_{R}=\mathfrak{g} \otimes_{k} R$ determines a map of $p$-restricted Lie algebras over $R$ where $R$ is a commutative $k$-algebra: $\mathfrak{g}_{a, R} \rightarrow \mathfrak{g}_{R}$. The corresponding map of height 1 infinitesimal group schemes $\mathbb{G}_{a(1), R} \rightarrow \underline{\mathfrak{g}}_{R}$ is the associated 1-parameter subgroup of $\mathfrak{g}$.

(2) $V\left(\mathbb{G}_{a(r)}\right) \simeq \mathbb{A}^{r}$. The $r$-tuple $\underline{a}=\left(a_{0}, \ldots, a_{r-1}\right) \in R^{\times r}=\mathbb{A}^{r}(R)$ corresponds to the 1-parameter subgroup $\mu_{\underline{a}}: \mathbb{G}_{a(r), R} \rightarrow \mathbb{G}_{a(r), R}$ whose map on coordinate algebras $R[T] / T^{p^{r}} \rightarrow R[T] / T^{p^{r}}$ sends $T$ to $\sum_{i} a_{i} T^{p^{i}}([25,1.10])$.

(3) By Proposition 1.11, $V\left(\mathrm{GL}_{n(r)}\right)=N_{p}^{[r]}\left(g l_{n}\right)$, the variety of $r$-tuples of pairwise commuting, $p$-nilpotent $n \times n$ matrices. The embedding $i_{r, r+1}: V_{r}\left(\mathrm{GL}_{n}\right) \simeq$ $N_{p}^{[r]}\left(g l_{n}\right) \subset V_{r+1}\left(\mathrm{GL}_{n}\right) \simeq N_{p}^{[r+1]}\left(g l_{n}\right)$ described in Remark [1.9 is given by sending an $r$-tuple $\left(\alpha_{0}, \ldots, \alpha_{r-1}\right)$ to the $(r+1)$-tuple $\left(0, \alpha_{0}, \ldots, \alpha_{r-1}\right)$. 
Let $X_{i, j}$ be the coordinate functions of $R\left[\mathrm{GL}_{n(r)}\right] \simeq R\left[X_{i, j}\right] /\left(X_{i, j}^{p^{r}}-\delta_{i, j}\right)$. Then $\exp _{\alpha}^{*}: R\left[\mathrm{GL}_{n(r)}\right] \rightarrow R\left[\mathbb{G}_{a(r)}\right]$ is given by sending $X_{i, j}$ for some $1 \leq i, j \leq n$ to the $(i, j)$-entry of the polynomial $p_{\underline{\alpha}}(t)$ with matrix coefficients whose coefficient of $t^{d}$ is computed as the multiple of $s^{d}$ in the $(i, j)$-entry of the matrix (1.10.1).

Upon performing the indicated multiplication in (1.10.1), the coefficient of $p_{\alpha}(t)$ multiplying $s^{p^{\ell}}$ is $\alpha_{\ell}$ for $0 \leq \ell<r$, whereas coefficients of $p_{\alpha}(t)$ multiplying $s^{n}$ for $n$ not a power of $p$ are determined as in formula (1.7.1). Consequently, we conclude that $\exp _{\underline{\alpha}}^{*}\left(X_{i, j}\right)$ is a polynomial in $t$ whose coefficient multiplying $T^{p^{\ell}}$ is $\left(\alpha_{\ell}\right)_{i, j}$ for $0 \leq \ell<\bar{r}$

(4) Since $\mathrm{SL}_{2(2)}$ is a group scheme with an embedding of exponential type (see [25. 1.8]), its variety admits a description similar to the one of $\mathrm{GL}_{n(r)}$. Namely, $V\left(\mathrm{SL}_{2(2)}\right)$ is the variety of pairs of $p$-nilpotent trace 0 proportional $2 \times 2$ matrices $\underline{\alpha}=\left(\alpha_{0}, \alpha_{1}\right)$. This variety is given explicitly as the affine scheme with coordinate algebra

$k\left[V\left(\mathrm{SL}_{2(2)}\right)\right]=k\left[x_{0}, y_{0}, z_{0}, x_{1}, y_{1}, z_{1}\right] /\left(x_{i} y_{i}-z_{i}^{2}, x_{0} y_{1}-x_{1} y_{1}, z_{0} y_{1}-z_{1} y_{0}, x_{0} z_{1}-x_{1} z_{0}\right)$.

We give an explicit description of the map on coordinate algebras

$$
\exp _{\underline{\alpha}}^{*}: R\left[\mathrm{SL}_{2(2)}\right] \rightarrow R\left[\mathbb{G}_{\mathrm{a}(2)}\right] \simeq R[T] / T^{p^{2}}
$$

induced by the one-parameter subgroup $\exp _{\underline{\alpha}}: \mathbb{G}_{a(2), R} \rightarrow \mathrm{SL}_{2(2), R}$. This description follows immediately from the general discussion in the previous example. Let $\underline{\alpha}=$ $\left(\left[\begin{array}{cc}c_{0} & a_{0} \\ b_{0} & -c_{0}\end{array}\right],\left[\begin{array}{cc}c_{1} & a_{1} \\ b_{1} & -c_{1}\end{array}\right]\right) \in N^{[2]}\left(s l_{2}\right)$. Then $\exp _{\underline{\alpha}}^{*}$ is determined by the formulae

$$
\begin{aligned}
& X_{1,1} \mapsto 1+c_{0} T+c_{1} T^{p}, \quad X_{1,2} \mapsto a_{0} T+a_{1} T^{p}, \\
& X_{2,1} \mapsto b_{0} T+b_{1} T^{p}, \quad X_{2,2} \mapsto 1-c_{0} T-c_{1} T^{p},
\end{aligned}
$$

where $X_{i, j}$ are the standard polynomial generators of $k\left[\mathrm{SL}_{2(2)}\right] \simeq \frac{k\left[X_{1,1}, X_{1,2}, X_{2,1}, X_{2,2}\right]}{\left(\operatorname{det}-1, X_{i, j}^{p^{2}}-\delta_{i, j}\right)}$.

Remark 1.13. If $k(v)$ denotes the field of definition of the point $v \in V(G)$ for an infinitesimal group scheme $G$ (see [26, p. 743] for a discussion of the field of definition), then we have a naturally associated map $\operatorname{Spec} k(v) \rightarrow V(G)$ and, hence, an associated group scheme homomorphism over $k(v)$ (for $r$ sufficiently large):

$$
\mu_{v}: \mathbb{G}_{a(r), k(v)} \longrightarrow G_{k(v)} .
$$

Note that if $K / k$ is a field extension and $\mu: \mathbb{G}_{a(r), K} \rightarrow G_{K}$ is a group scheme homomorphism, then this data defines a point $v \in V(G)$ and a field embedding $k(v) \hookrightarrow K$ such that $\mu$ is obtained from $\mu_{v}$ via scalar extension from $k(v)$ to $K$.

We next recall the rank variety and cohomological support variety of a $k G$-module of an infinitesimal group scheme. We use the notation

$$
\mathrm{H}^{\bullet}(G, k)= \begin{cases}\mathrm{H}^{*}(G, k), & \text { if } p=2, \\ \mathrm{H}^{\mathrm{ev}}(G, k) & \text { if } p>2 .\end{cases}
$$

Definition 1.14. Let $G$ be a finite group scheme and $M$ a finite dimensional $k G$-module. We define the cohomological support variety for $M$ to be

$$
|G|_{M} \equiv V\left(\operatorname{ann}_{\mathrm{H} \cdot(G, k)} \operatorname{Ext}_{k G}^{*}(M, M)\right),
$$

the reduced closed subscheme of $|G|=\operatorname{Spec~}^{\bullet}(G, k)_{\text {red }}$ given as the variety of the annihilator ideal of $\operatorname{Ext}_{k G}^{*}(M, M)$. 
The map of $R$-algebras (but not of Hopf algebras for $r>1$ ),

$$
\epsilon: R[u] / u^{p} \stackrel{u \mapsto u_{r-1}}{\longrightarrow} R\left[u_{0}, \ldots, u_{r-1}\right] /\left(u_{i}^{p}\right) \simeq R \mathbb{G}_{a(r)},
$$

makes its first appearance in the following definition and will recur throughout this paper.

Definition 1.15. Let $G$ be an infinitesimal group scheme and $M$ a finite dimensional $k G$-module. We define the rank variety for $M$ to be the reduced closed subscheme $V(G)_{M}$ whose points are given as follows:

$$
V(G)_{M}=\left\{v \in V(G):\left(\mu_{v, *} \circ \epsilon\right)^{*}\left(M_{k(v)}\right) \text { is not free as a } k[u] / u^{p} \text {-module }\right\} .
$$

Proposition [26, 6.2] asserts that $V(G)_{M}$ is a closed subvariety of $V(G)$. A key result of [26] is the following theorem relating the scheme of 1-parameter subgroups $V(G)$ to the cohomology of $G$.

Theorem 1.16 ([26, 5.2, 6.8, 7.5]). Let $G$ be an infinitesimal group scheme of height $\leq r$. There is a natural homomorphism of $k$-algebras,

$$
\psi: \mathrm{H}^{\bullet}(G, k) \rightarrow k[V(G)]
$$

with nilpotent kernel whose image contains the $p^{r}$-th power of each element of $k[V(G)]$. Hence, the associated morphism of schemes,

$$
\Psi: V(G) \rightarrow \operatorname{Spec~H}^{\bullet}(G, k)
$$

is a p-isogeny.

If $M$ is a finite dimensional $k G$-module, then $\Psi$ restricts to a homeomorphism

$$
\Psi_{M}: V(G)_{M} \stackrel{\sim}{\longrightarrow}|G|_{M} .
$$

Furthermore, every closed conical subspace of $V(G)$ is of the form $V(G)_{M}$ for some finite dimensional $k G$-module $M$.

In the special case of $G=\mathrm{GL}_{n(r)}$ the isogeny $\Psi$ has an explicitly constructed "inverse."

Theorem 1.17 ([25, 5.2]). There exists a homomorphism of $k$-algebras,

$$
\phi: k\left[V\left(\mathrm{GL}_{n(r)}\right)\right] \rightarrow \mathrm{H}^{\bullet}\left(\mathrm{GL}_{n(r)}, k\right),
$$

such that $\psi \circ \phi$ is the $r^{\text {th }}$ iterate of the $k$-linear Frobenius map. Hence, the associated morphisms of schemes,

$$
\Psi: V\left(\mathrm{GL}_{n(r)}\right) \rightarrow \operatorname{Spec~H}^{\bullet}\left(\mathrm{GL}_{n(r)}, k\right), \quad \Phi: \mathrm{Spec}^{\bullet}\left(\mathrm{GL}_{n(r)}, k\right) \rightarrow V\left(\mathrm{GL}_{n(r)}\right),
$$

are mutually inverse homeomorphisms.

Example 1.18. We investigate $V(G)_{M}$ for the four examples of Example 1.5.

(1) Let $M$ be a $p$-restricted $\mathfrak{g}$-module of dimension $m$, given by the map of $p$ restricted Lie algebras $\rho: \mathfrak{g} \rightarrow \operatorname{End}_{k}(M) \simeq \mathfrak{g l}_{m}$. Then $V(\underline{\mathfrak{g}})_{M} \subset V\left(\underline{\mathfrak{g l}}_{m}\right)$ consists of those $p$-nilpotent elements of $\mathfrak{g}$ whose Jordan type (as an $m \times m$-matrix in $\mathfrak{g l}_{m}$ ) has at least one block of size $<p$ (see [13]).

(2) For $G=\mathbb{G}_{a(r)}, k G \simeq k E$, where $E$ is an elementary abelian $p$-group of rank $r$. The rank variety of a $k E$-module was first investigated in [6].

We consider directly the rank variety $V\left(\mathbb{G}_{a(r)}\right)_{M}$ of a finite dimensional $k \mathbb{G}_{a(r)^{-}}$ module $M$. The data of such a module is the choice of $r p$-nilpotent, pairwise 
commuting endomorphisms $\widetilde{u}_{0}, \ldots, \widetilde{u}_{r-1} \in \operatorname{End}_{k}(M)$, given as the image of the distinguished generators of $k \mathbb{G}_{a(r)}$ as in (1.5.1). A 1-parameter subgroup of $\mathbb{G}_{a(r)}$ has the form $\mu_{\underline{a}}: \mathbb{G}_{a(r), K} \rightarrow \mathbb{G}_{a(r), K}$ for some $r$-tuple $\underline{a}=\left(a_{0}, \ldots, a_{r-1}\right)$ of $K$-rational points as in Example $1.12(2)$. The condition that $\mu_{\underline{a}}$ be a point of $V\left(\mathbb{G}_{a(r)}\right)_{M}$ is the condition that $\left(\mu_{a} \circ \epsilon\right)^{*}\left(M_{K}\right)$ is not free as a $\bar{K}[u] / u^{p}$-module, which is equivalent to the condition that $M_{K}$ is not free as a $K[\widetilde{u}] / \widetilde{u}^{p}$-module where $\widetilde{u}=a_{r-1} \widetilde{u}_{0}+a_{r-2}^{p} \widetilde{u}_{1}+\cdots+a_{0}^{p^{r-1}} \widetilde{u}_{r-1} \in \operatorname{End}_{K}\left(M_{K}\right)$ (see [26, 6.5]).

(3) Let $M$ be a finite dimensional $k G$-module with $G=\mathrm{GL}_{n(r)}$. By Theorem 1.16. $V\left(\mathrm{GL}_{n(r)}\right)_{M} \subset V\left(\mathrm{GL}_{n(r)}\right)$ is the closed subvariety whose set of points in a field $K / k$ are 1-parameter subgroups $\exp _{\underline{\alpha}}: \mathbb{G}_{a(r), K} \rightarrow \mathrm{GL}_{n(r), K}$ indexed by $r$ tuples $\underline{\alpha}=\left(\alpha_{0}, \ldots, \alpha_{r-1}\right) \in M_{n}(K)$ of $p$-nilpotent, pairwise commuting matrices such that $\left(\exp _{\underline{\alpha}, *} \circ \epsilon\right)^{*}\left(M_{K}\right)$ is not free as a $K[u] / u^{p}$-module. The action of $u$ on $M_{K}$ is determined utilizing Example 1.12(3). Namely, the action of $u$ is given by composing the coproduct $M_{K} \rightarrow K\left[\mathrm{GL}_{n(r)}\right] \otimes M_{K}$ defining the $\mathrm{GL}_{n(r)}$-module structure on $M_{K}$ with the linear functional

$$
u_{r-1} \circ \exp _{\underline{\alpha}}^{*}: K\left[\mathrm{GL}_{n(r)}\right] \stackrel{\exp _{\underline{\alpha}}^{*}}{\longrightarrow} K\left[\mathbb{G}_{a(r)}\right] \stackrel{u_{r-1}}{\longrightarrow} K .
$$

In Section 3, we shall investigate this case in more detail by considering some concrete examples.

(4) A complete description of support varieties for simple modules for $\mathrm{SL}_{2(r)}$ can be found in [26, 87 . We describe the situation for $G=\mathrm{SL}_{2(2)}$. Let $S_{\lambda}$ be irreducible modules of highest weight $\lambda$, where $0 \leq \lambda \leq p^{2}-1$. For $\lambda<p-1$, the module $S_{\lambda}$ has dimension less than $p$, and thus $V(G)_{S_{\lambda}}=V(G)_{S_{\lambda}^{(1)}}=V(G)$. Here, $S_{\lambda}^{(i)}$ is the $i^{\text {th }}$ Frobenius twist of $S_{\lambda}$. For $\lambda=p-1$, the restriction of $S_{p-1}$ to $\mathrm{SL}_{2(1)} \subset \mathrm{SL}_{2(2)}$ is projective (the Steinberg module for $\mathrm{SL}_{2(1)}$ ), but $S_{p-1}$ is not itself projective. Hence, $V(G)_{S_{p-1}}$ is a proper non-trivial subvariety of $V(G)$. Using the notation introduced in Example 1.12(4), we have

$$
V(G)_{S_{p-1}}=\left\{\left(\alpha_{0}, 0\right) \mid \alpha_{0} \in N\left(s l_{2}\right)\right\} \subset V(G)
$$

and

$$
V(G)_{S_{p-1}^{(1)}}=\left\{\left(0, \alpha_{1}\right) \mid \alpha_{1} \in N\left(s l_{2}\right)\right\} \subset V(G)
$$

(see [26, 6.10]). $V(G)_{S_{p-1}}$ can be described as the subscheme of $V(G)$ defined by the equations $x_{1}=y_{1}=z_{1}=0$. For $\lambda=\lambda_{0}+\lambda_{1} p$ where $\lambda_{0}, \lambda_{1} \leq p-1$ we have $S_{\lambda} \simeq S_{\lambda_{0}} \otimes S_{\lambda_{1}}^{(1)}$ by the Steinberg tensor product theorem. Hence, we can compute the support variety of $S_{\lambda}$ using the tensor product property of support varieties. For $\lambda=p^{2}-1, S_{\lambda}$ is the Steinberg module for $\mathrm{SL}_{2(2)}$, it is projective and, hence, $V(G)_{S_{p^{2}-1}}=\{0\}$. Overall, we get

$$
V(G)_{S_{\lambda}}= \begin{cases}N^{[2]}\left(s l_{2}\right) & \text { if } \lambda_{0}, \lambda_{1} \neq p-1 \\ \left\{\left(\alpha_{0}, 0\right) \mid \alpha_{0} \in N\left(s l_{2}\right)\right\} & \text { if } \lambda_{0}=p-1, \lambda_{1} \neq p-1, \\ \left\{\left(0, \alpha_{1}\right) \mid \alpha_{1} \in N\left(s l_{2}\right)\right\} & \text { if } \lambda_{0} \neq p-1, \lambda_{1}=p-1 \\ 0 & \text { if } \lambda=p^{2}-1\end{cases}
$$




\section{Global $p$-Nilpotent operators}

In this section, we introduce in Definition 2.1 and study the global $p$-nilpotent operator

$$
\Theta_{G}: k[G] \rightarrow k[V(G)]
$$

a $k$-linear but not multiplicative map defined for any infinitesimal group scheme $G$. This operator, when viewed as an element of $k G \otimes k[V(G)]$, encodes all 1-parameter subgroups of $G$ : any 1-parameter subgroup $\mu: \mathbb{G}_{a(r), K} \rightarrow G_{K}$ corresponds to a $K$-valued point of $V(G)$, and $\mu_{*}\left(u_{r-1}\right)$ equals the specialization in $K G$ of $\Theta_{G} \in$ $k G \otimes k[V(G)]$ at this point.

If $M$ is a rational $G$-module, then $\Theta_{G}$ determines the $k[V(G)]$-linear operator

$$
\Theta_{M}: M \otimes k[V(G)] \rightarrow M \otimes k[V(G)]
$$

as formalized in Definition 2.3 .

Before giving definitions, we mention as motivation the example $G=\mathbb{G}_{a(1)}^{\times 2}$. In this case, the group algebra $k G$ equals $k[x, y] /\left(x^{p}, y^{p}\right)$, the scheme of 1-parameter subgroups equals $V(G)=\mathbb{A}^{2}$, and $k[V(G)]=k\left[\mathbb{A}^{2}\right]=k[s, t]$. In this special case, $\Theta_{G}$ takes the form

$$
\Theta_{G}=x \otimes s+y \otimes t \in k[x, y] /\left(x^{p}, y^{p}\right) \otimes k[s, t] .
$$

If $M$ is a $k G$-module, then the $k[s, t]$-linear operator $\Theta_{M}$ is given by

$$
\begin{gathered}
\Theta_{M}: M \otimes k[s, t] \rightarrow M \otimes k[s, t], \\
m \otimes 1 \mapsto x m \otimes s+y m \otimes t .
\end{gathered}
$$

"Specializing" $\Theta_{M}$ at some $(a, b) \in K^{2}$ for some field extension $K / k$ yields the action of $a x+b y$ on $M_{K}$.

To construct our global operator, we proceed as follows. Let $G$ be an algebraic affine group scheme over $k$ (that is, $G$ is an affine group scheme such that the coordinate algebra $k[G]$ is finitely generated over $k$ [27, 3.3]) and let $A=k\left[V_{r}(G)\right]$. The natural isomorphism of covariant functors on commutative $k$-algebras $R$,

$$
\operatorname{Hom}_{\operatorname{grp~sch}}\left(\mathbb{G}_{a(r), R}, G_{R}\right) \simeq \operatorname{Hom}_{k-\operatorname{alg}}(A, R),
$$

given in Theorem 1.8 implies the existence of a universal 1-parameter subgroup of height $r$,

$$
\mathcal{U}_{G, r}: \mathbb{G}_{a(r), A} \longrightarrow G_{A},
$$

the subgroup corresponding to the identity map on $A$. The subgroup $\mathcal{U}_{G, r}$ induces a map on coordinate algebras

$$
\mathcal{U}_{G, r}^{*}: A \otimes k[G] \longrightarrow A \otimes k\left[\mathbb{G}_{a(r)}\right] .
$$

Recall that $k \mathbb{G}_{a(r)} \simeq k\left[u_{0}, \ldots, u_{r-1}\right] /\left(u_{0}^{p}, \ldots, u_{r-1}^{p}\right)$, where $u_{r-1}$, the dual element to $T^{p^{r-1}}$, is a linear functional $u_{r-1}: k\left[\mathbb{G}_{a(r)}\right] \rightarrow k$.

Definition 2.1. Let $G$ be an algebraic affine group scheme over $k$. We define

$$
\Theta_{G, r}: k[G] \longrightarrow k\left[V_{r}(G)\right]
$$

to be the $k$-linear, $p$-nilpotent functional defined by the composition

$$
k[G] \stackrel{1 \otimes \mathrm{id}}{\longrightarrow} k\left[V_{r}(G)\right] \otimes k[G] \stackrel{\mathcal{U}_{G, r}^{*}}{\longrightarrow} k\left[V_{r}(G)\right] \otimes k\left[\mathbb{G}_{a(r)}\right] \stackrel{\mathrm{id} \otimes u_{r-1}}{\longrightarrow} k\left[V_{r}(G)\right] .
$$


As an element of $\operatorname{Hom}\left(k[G], k\left[V_{r}(G)\right]\right) \equiv k G \otimes k\left[V_{r}(G)\right], \Theta_{G, r}$ can be equivalently defined as

$$
\Theta_{G, r}=\mathcal{U}_{G, r, *}\left(u_{r-1}\right) \in k G \otimes k\left[V_{r}(G)\right]
$$

where

$$
\mathcal{U}_{G, r, *}: k \mathbb{G}_{a(r)} \otimes k\left[V_{r}(G)\right] \rightarrow k G \otimes k\left[V_{r}(G)\right]
$$

Thus $\Theta_{G, r}$, as given in (2.1.1), satisfies the property that its composition with $k\left[V_{r}(G)\right] \rightarrow K$ corresponding to the $K$-rational point $\mu: \mathbb{G}_{a(r), K} \rightarrow G_{K}$ equals $\mu_{*}\left(u_{r-1}\right)$.

The following proposition justifies using the simplified notation

$$
\Theta_{G}: k[G] \rightarrow k[V(G)]
$$

where $V(G)=\lim _{\longrightarrow} V_{r}(G)$ as in Definition 1.10. Namely, $\Theta_{G}$ is defined to be isomorphic to $\Theta_{G, r}$ provided that $G$ is infinitesimal of height $\leq r$.

Recall the canonical projection $p_{r^{\prime}, r}: \mathbb{G}_{a\left(r^{\prime}\right)} \rightarrow \mathbb{G}_{a(r)}$, and the induced closed embedding $i_{r, r^{\prime}}: V_{r}(G) \hookrightarrow V_{r^{\prime}}(G)$, introduced in Remark [1.9, for $r^{\prime} \geq r$.

Proposition 2.2. Let $G$ be an infinitesimal group scheme and let $r^{\prime} \geq r$. Let $A_{r}=k\left[V_{r}(G)\right], A_{r^{\prime}}=k\left[V_{r^{\prime}}(G)\right]$, and let

$$
\phi: A_{r^{\prime}} \rightarrow A_{r}
$$

be the surjective homomorphism corresponding to the canonical embedding $i_{r, r^{\prime}}$ : $V_{r}(G) \hookrightarrow V_{r^{\prime}}(G)$. Consider $A_{r}$ as an $A_{r^{\prime}}$-module via $\phi$. Then

$$
\Theta_{G, r}=\Theta_{G, r^{\prime}} \otimes_{A_{r^{\prime}}} 1 \in k G \otimes A_{r^{\prime}} \otimes_{A_{r^{\prime}}} A_{r} \simeq k G \otimes A_{r} .
$$

Moreover, if $G$ is an infinitesimal group scheme of height $\leq r$, then $\Theta_{G, r}$ is thereby naturally identified with $\Theta_{G, r^{\prime}}$.

Proof. Consider the composition

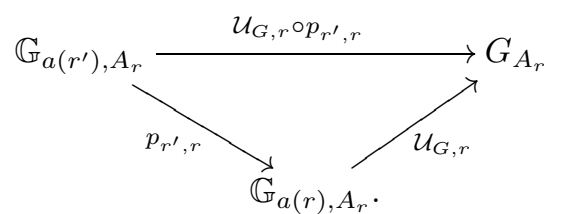

Since $\mathcal{U}_{G, r} \in V_{r}(G)\left(A_{r}\right) \simeq \operatorname{Hom}\left(A_{r}, A_{r}\right)$ corresponds to the identity map on $A_{r}$ and $p_{r^{\prime}, r}$ is the map that induces $\phi: A_{r^{\prime}} \rightarrow A_{r}$, we conclude that the composition $\mathcal{U}_{G, r} \circ p_{r^{\prime}, r} \in V_{r^{\prime}}(G) \simeq \operatorname{Hom}\left(A_{r^{\prime}}, A_{r}\right)$ corresponds to $\phi$. Hence, the universality of $\mathcal{U}_{G, r^{\prime}}$ implies that $\mathcal{U}_{G, r} \circ p_{r^{\prime}, r}$ is obtained by pushing down the universal 1-parameter subgroup $\mathcal{U}_{G, r^{\prime}}$ via $\phi: A_{r^{\prime}} \rightarrow A_{r}$. Therefore, we conclude that

$$
\mathcal{U}_{G, r} \circ p_{r^{\prime}, r}=\mathcal{U}_{G, r^{\prime}} \otimes_{A_{r^{\prime}}} A_{r}
$$

which implies the equality of maps of group algebras

$$
\mathcal{U}_{G, r^{\prime}, *} \otimes_{A_{r^{\prime}}} A_{r}=\mathcal{U}_{G, r, *} \circ p_{r^{\prime}, r, *}: k \mathbb{G}_{a\left(r^{\prime}\right)} \otimes A_{r} \rightarrow k G \otimes A_{r} .
$$

Since $p_{r^{\prime}, r, *}\left(u_{r^{\prime}-1}\right)=u_{r-1} \in k \mathbb{G}_{a(r)}$, we conclude that

$$
\left(\mathcal{U}_{G, r, *} \circ p_{r^{\prime}, r, *}\right)\left(u_{r^{\prime}-1}\right)=\mathcal{U}_{G, r, *}\left(u_{r-1}\right)=\Theta_{G, r},
$$

whereas $\left(\mathcal{U}_{G, r^{\prime}, *} \otimes_{A_{r^{\prime}}} A_{r}\right)\left(u_{r^{\prime}-1}\right)=\mathcal{U}_{G, r^{\prime}, *}\left(u_{r^{\prime}-1}\right) \otimes_{A_{r^{\prime}-1}} 1=\Theta_{G, r^{\prime}} \otimes_{A_{r^{\prime}}} 1$.

The second statement follows immediately from the fact that for $G$ of height $\leq r$, the map $\phi: A_{r^{\prime}} \rightarrow A_{r}$ is an isomorphism as shown in Remark 1.9. 
Let $G$ be an affine group scheme over $k, M$ be a $k G$-module, and $\nabla_{M}: M \rightarrow$ $M \otimes k[G]$ be the corresponding co-action. A $k$-linear functional with values in a commutative $k$-algebra $A, \Theta: k[G] \rightarrow A$, determines an action of $\Theta$ on $M \otimes A$ which is the $A$-linear extension

$$
\Theta_{M}: M \otimes A \rightarrow M \otimes A
$$

of the map

$$
M \stackrel{\nabla_{M}}{\longrightarrow} M \otimes k[G] \stackrel{\text { id } \otimes \Theta_{M}}{\longrightarrow} M \otimes A .
$$

If $G$ is finite, we may view $\Theta \in \operatorname{Hom}_{k}(k[G], A$ ) as an element of $k G \otimes A$ (which we also denote by $\Theta$ ). From this point of view, the action (2.2.4) is simply multiplication by $\Theta$.

We now define the global p-nilpotent operator on a $G$-module $M$.

Definition 2.3. Let $G$ be an infinitesimal group scheme. For any $k[G]$-comodule $M$ with co-action $\nabla_{M}: M \rightarrow M \otimes k[G]$, we define the $p$-nilpotent operator

$$
\Theta_{M}: M \otimes k[V(G)] \rightarrow M \otimes k[V(G)]
$$

to be the $k[V(G)]$-linear extension of the $\operatorname{map}\left(\operatorname{id}_{M} \otimes \Theta_{G}\right) \circ \nabla_{M}: M \rightarrow M \otimes k[V(G)]$.

Remark 2.4. The fact that $\Theta_{M}$ is $p$-nilpotent follows immediately from (2.1.2) since $u_{r-1}^{p}=0$.

Slightly abusing notation, we shall often refer to $\Theta_{G}$ itself as the global $p$ nilpotent operator.

We reformulate the pairing (2.2.4) in a more geometric fashion as follows.

Proposition 2.5. Let $G$ be a group scheme over $k, V$ be an affine $k$-scheme, and let $M$ be a finite dimensional $G$-module. Then a k-linear functional $\Theta: k[G] \rightarrow k[V]$ determines the pairing of $k$-schemes

$$
V \times M \rightarrow M .
$$

As a pairing of representable functors of commutative $k$-algebras $A$, this pairing sends $\left(v: k[V] \rightarrow A, m=\sum_{i} a_{i} \otimes m_{i}\right)$ to $\sum_{i} a_{i}\left(\sum_{j} v\left(\Theta\left(f_{i, j}\right)\right) m_{j}\right)$, where $\nabla\left(m_{i}\right)=$ $\sum_{j} f_{i, j} \otimes m_{j}$.

Example 2.6. We describe the global $p$-nilpotent operator $\Theta_{G}$ in each of the four examples of Example 1.5.

(1) Let $G=\mathrm{GL}_{m(1)} \equiv \underline{\mathfrak{g l}}_{m}$, with group algebra $k \underline{\mathfrak{g l}}_{m}=u\left(\mathfrak{g l}_{m}\right)$. Then the composition of

$$
\Theta_{G}: k[G]=k\left[X_{i, j}\right] /\left(X_{i, j}^{p}-\delta_{i, j}\right) \rightarrow k\left[N_{p}\left(\mathfrak{g l}_{m}\right)\right]
$$

with some $K$-rational point $x \in N_{p}\left(\mathfrak{g l}_{m}\right)$ is the evaluation of the matrix coordinate functions $X_{i, j}$ on $x$. In other words,

$$
\Theta_{\underline{\mathfrak{g l}}_{m}}=\sum_{1 \leq i, j \leq m} x_{i, j} \otimes \bar{X}_{i, j} \in \mathfrak{u}\left(\mathfrak{g l}_{m}\right) \otimes k\left[N_{p}\left(\mathfrak{g l}_{m}\right)\right],
$$

where $\bar{X}_{i, j}$ is the image of the $i, j$-matrix coefficient on $\mathfrak{g l}_{m}$.

For a general $p$-restricted Lie algebra $\mathfrak{g}$, we have

$$
\Theta_{\underline{\mathfrak{g}}}=\sum x \otimes \bar{X} \in \mathfrak{u}(\mathfrak{g}) \otimes k\left[N_{p}(\mathfrak{g})\right],
$$


where the sum is over basis elements $X \in \mathfrak{g}^{\#}$ with image $\bar{X} \in k\left[N_{p}(\mathfrak{g})\right]$ and with dual $x \in \mathfrak{g}$.

We record an explicit formula for the universal $p$-nilpotent operator in the case of $\mathfrak{g}=s l_{2}$ for future reference. We have $k\left[N_{p}\left(s l_{2}\right)\right] \simeq k[x, y, z] /\left(x y+z^{2}\right)$. Let $e, f, h$ be the standard basis of the $p$-restricted Lie algebra $s l_{2}$. Then

$$
\Theta_{\underline{s} l_{2}}=x e+y f+z h .
$$

Observe that this formula agrees with the presentation of a "generic" $\pi$-point for $u\left(s l_{2}\right)$ as given in [17, 2.5].

(2) Take $G=\mathbb{G}_{a(r)}$. Then $k\left[\mathbb{G}_{a(r)}\right] \simeq k[T] / T^{p^{r}}$, and $k\left[V\left(\mathbb{G}_{a(r)}\right)\right] \simeq k\left[x_{0}, \ldots, x_{r-1}\right]$ is graded in such a way that $x_{i}$ has degree $p^{i}$ (see Proposition 2.11 below). We compute $\Theta_{\mathbb{G}_{a(r)}}: k\left[\mathbb{G}_{a(r)}\right] \longrightarrow k\left[V\left(\mathbb{G}_{a(r)}\right)\right]$ explicitly in this case (see also [26, 6.5.1]).

1-parameter subgroups of $\mathbb{G}_{a(r), K}$ are in one-to-one correspondence with the additive polynomials in $K[T] / T^{p^{r}}$, that is, polynomials of the form $p(T)=a_{0} T+$ $a_{1} T^{p}+\cdots+a_{r-1} T^{p^{r-1}}$ (see [25, 1.10]). The map on coordinate algebras induced by the universal 1-parameter subgroup $\mathcal{U}: \mathbb{G}_{a(r), k[V(G)]} \rightarrow \mathbb{G}_{a(r), k[V(G)]}$ is given by the "generic" additive polynomial:

$$
\begin{gathered}
\mathcal{U}^{*}: k\left[x_{0}, \ldots, x_{r-1}\right][T] / T^{p^{r}} \longrightarrow k\left[x_{0}, \ldots, x_{r-1}\right][T] / T^{p^{r}}, \\
T \mapsto x_{0} T+x_{1} T^{p}+\cdots+x_{r-1} T^{p^{r-1}} .
\end{gathered}
$$

To determine the linear functional

$$
\Theta_{\mathbb{G}_{a(r)}}=u_{r-1} \circ \mathcal{U}^{*}: k[T] / T^{p^{r}} \longrightarrow k\left[x_{0}, \ldots, x_{r-1}\right],
$$

it suffices to determine the values of $\Theta_{\mathbb{G}_{a(r)}}$ on the linear generators $\left\{T^{i}\right\}, 0 \leq i \leq$ $p^{r}-1$. Since $u_{r-1}$ is the dual to $T^{p^{r-1}}$, this further reduces to determining the coefficient by $T^{p^{r-1}}$ in $\mathcal{U}^{*}\left(T^{i}\right)=\left(x_{0} T+x_{1} T^{p}+\cdots+x_{r-1} T^{p^{r-1}}\right)^{i}$. Computing this coefficient, we conclude that $\Theta_{\mathbb{G}_{a(r)}}$ is given explicitly on the basis elements of $k\left[\mathbb{G}_{a(r)}\right] \simeq k[T] / T^{p^{r}}$ by

$$
T^{i} \mapsto \sum_{\substack{i_{0}+i_{1}+\cdots+i_{r-1}=i \\
i_{0}+i_{1} p+\cdots+i_{r-1} p^{r-1}=p^{r-1}}}\left(\begin{array}{c}
i \\
i_{0}, i_{1}, \ldots, i_{r-1}
\end{array}\right) x_{0}^{i_{0}} \ldots x_{r-1}^{i_{r-1}},
$$

where $\left(\begin{array}{c}i \\ i_{0}, i_{1}, \ldots, i_{r-1}\end{array}\right)=\frac{i !}{i_{0} ! i_{1} ! \ldots i_{r-1} !}$ is the multinomial coefficient. Let $\left\{v_{0}, \ldots, v_{p^{r}-1}\right\}$ be the linear basis of $k \mathbb{G}_{a(r)}$ dual to $\left\{1, T, \ldots, T^{p^{r}-1}\right\}$. Dualizing (2.6.2), we conclude that $\Theta_{\mathbb{G}_{a(r)}}$, as an element of $k \mathbb{G}_{a(r)} \otimes k\left[V\left(\mathbb{G}_{a(r)}\right)\right]$, has the following form:

$$
\Theta_{\mathbb{G}_{a(r)}}=\sum_{i=0}^{p^{r}-1} v_{i}\left[\sum_{\substack{i_{0}+i_{1}+\cdots+i_{r-1}=i \\
i_{0}+i_{1} p+\cdots+i_{r-1} p^{r-1}=p^{r-1}}}\left(\begin{array}{c}
i \\
i_{0}, i_{1}, \ldots, i_{r-1}
\end{array}\right) x_{0}^{i_{0}} \cdots x_{r-1}^{i_{r-1}}\right] .
$$

By [25, 1.4], $v_{i}$ can be expressed in terms of the algebraic generators $u_{j}$ of $k \mathbb{G}_{a(r)}$ via the following formulae:

$$
v_{i}=\frac{u_{0}^{j_{0}} \cdots u_{r-1}^{j_{r-1}}}{j_{0} ! \cdots j_{r-1} !}
$$

where $i=j_{0}+j_{1} p+\cdots+j_{r-1} p^{r-1}\left(0 \leq j_{\ell} \leq p-1\right)$ is the $p$-adic expansion of $i$. 
Using these formulae, it is straightforward to calculate the term of $\Theta_{\mathbb{G}_{a(r)}}$ which is linear with respect to $u_{i}$ (and homogeneous of degree $p^{r-1}$ with respect to the grading of $\left.k\left[V\left(\mathbb{G}_{a(r)}\right)\right]\right)$ :

$$
u_{0} x_{r-1}+u_{1} x_{r-2}^{p}+\cdots+u_{r-1} x_{0}^{p^{r-1}}
$$

The "linear" term gives the entire operator $\Theta_{\mathbb{G}_{a(r)}}$ for $r=1,2$, but for $r \geq 3$, the non-linear terms start to appear.

(3) Let $G=\mathrm{GL}_{n(r)}$. Recall that $V\left(\mathrm{GL}_{n(r)}\right)$ is the $k$-scheme of $r$-tuples of $p$ nilpotent, pairwise commuting matrices. For notational convenience, let $A$ denote $k\left[V\left(\mathrm{GL}_{n(r)}\right)\right]=k\left[M_{n}^{\times r}\right] / I$, a quotient of the coordinate algebra of the variety $M_{n}^{\times r}$ of $r$-tuples of $n \times n$ matrices. Then $\mathcal{U}_{\mathrm{GL}_{n(r)}}: \mathbb{G}_{a(r), A} \rightarrow \mathrm{GL}_{n(r), A}$ is specified by the $A$-linear map on coordinate algebras,

$$
\mathcal{U}_{\mathrm{GL}_{n(r)}}^{*}: A\left[\mathrm{GL}_{n(r)}\right] \rightarrow A[T] / T^{p^{r}}, \quad X_{i, j} \mapsto \sum_{\ell=0}^{p^{r}-1}\left(\beta_{\ell}\right)_{i, j} T^{j}
$$

where $\left\{X_{i, j} ; 1 \leq i, j \leq n\right\}$ are the matrix coordinate functions of $\mathrm{GL}_{n}$, where $\beta_{\ell}$ is given as in formula (1.7.1) in terms of the matrices $\alpha_{0}, \ldots, \alpha_{r-1} \in M_{n}(A)$ and $\alpha_{i}=\beta_{p^{i}}$ have matrix coordinate functions which generate $A$. (Indeed, the $n^{2} r$ entries of $\alpha_{0}, \ldots, \alpha_{r-1}$ viewed as variables generate $A$, with relations given by the conditions that these matrices must be $p$-nilpotent and pairwise commuting.)

The $p$-nilpotent operator

$$
\Theta_{\mathrm{GL}_{n(r)}}: k\left[\mathrm{GL}_{n(r)}\right] \rightarrow k\left[V\left(\mathrm{GL}_{n(r)}\right)\right]
$$

is given by the $k$-linear functional sending a polynomial in the matrix coefficients $P\left(X_{i, j}\right) \in k\left[\mathrm{GL}_{n(r)}\right]$ to the coefficient of $T^{p^{r-1}}$ of the sum of products corresponding to the polynomial $P$ given by replacing each $X_{i, j}$ by $\sum_{\ell=0}^{p^{r}-1}\left(\beta_{\ell}\right)_{i, j} T^{\ell}$ (when taking products of matrix coefficients, one uses the usual rule for matrix multiplication);

$$
P\left(X_{i, j}\right) \mapsto P\left(\sum_{\ell=0}^{p^{r}-1}\left(\beta_{\ell}\right)_{i, j} T^{\ell}\right) \mapsto \text { coeff of } T^{p^{r-1}}
$$

For example, the coaction $k^{n} \rightarrow k\left[\mathrm{GL}_{n}\right] \otimes k^{n}$ corresponding to the natural representation of $\mathrm{GL}_{n}$ on $k^{n}$ determines an action of

$$
\operatorname{Hom}_{k}\left(k\left[\mathrm{GL}_{n(r)}\right], A\right) \subset \operatorname{Hom}_{k}\left(k\left[\mathrm{GL}_{n}\right], A\right)
$$

on $A^{n}$, so that $\Theta_{\mathrm{GL}_{n(r)}}: A^{n} \rightarrow A^{n}$ is given in matrix form by $\left(\Theta_{\mathrm{GL}_{n(r)}}\left(X_{i, j}\right)\right)$.

(4) We consider $G=\mathrm{SL}_{2(2)}$, and assume notation and conventions of Example $1.12(4)$. Let $A=k\left[\mathrm{SL}_{2(2)}\right]$. Using the general discussion in Example [2.6 $(3)$ above (also compare to Example $1.12(4)$ ), one readily computes that the map on coordinate algebras $\mathcal{U}_{\mathrm{SL}_{2(2)}}^{*}: A\left[\mathrm{SL}_{2(2)}\right] \rightarrow A\left[\mathbb{G}_{a(2)}\right] \simeq A[T] / T^{p^{2}}$ is given by

$$
\begin{gathered}
X_{1,1} \mapsto 1+z_{0} T+z_{1} T^{p}, \quad X_{1,2} \mapsto x_{0} T+x_{1} T^{p} \\
X_{2,1} \mapsto y_{0} T+y_{1} T^{p}, \quad X_{2,2} \mapsto 1-z_{0}-z_{1} T^{p}
\end{gathered}
$$

By (2.1.2), $\Theta_{\mathrm{SL}_{2(2)}}=\mathcal{U}_{\mathrm{SL}_{2(2)} *}\left(u_{1}\right)$, which is the functional given by "reading off the coefficient of $T^{p}$ ". 
Let $e, f, h, e^{(p)}, f^{(p)}, h^{(p)} \in k \mathrm{SL}_{2(2)}$ denote respectively the linear duals of the functions $X_{1,2}, X_{2,1}, X_{1,1}-1, X_{1,2}^{p}, X_{2,1}^{p},\left(X_{1,1}-1\right)^{p}$ on $\mathrm{SL}_{2(2)}$, and set

$$
e^{(i)}=\frac{e^{i}}{i !}, \quad f^{(i)}=\frac{f^{i}}{i !}, \quad\left(\begin{array}{c}
h \\
i
\end{array}\right)=\frac{h(h-1)(h-2) \ldots(h-i+1)}{i !}
$$

for $i<p$. Fix the linear basis of $k\left[\mathrm{SL}_{2(2)}\right]$ given by powers of $X_{1,2}, X_{2,1}, X_{1,1}-1$ (in this fixed order). Then the element of $k \mathrm{SL}_{2(2)}$ dual to $X_{1,2}^{i} X_{2,1}^{j}\left(X_{1,1}-1\right)^{\ell}$ for $i+j+\ell \leq p$ is given by

$$
\left(X_{1,2}^{i} X_{2,1}^{j}\left(X_{1,1}-1\right)^{\ell}\right)^{\#}=e^{(i)} f^{(j)}\left(\begin{array}{l}
h \\
\ell
\end{array}\right)
$$

(where $\left(\begin{array}{l}h \\ p\end{array}\right)$ is identified with $h^{(p)}$ by definition).

With these conventions $\Theta_{\mathrm{SL}_{2(2)}} \in k \mathrm{SL}_{2(2)} \otimes k\left[V\left(\mathrm{SL}_{2(2)}\right)\right]$ equals

$$
x_{1} e+y_{1} f+z_{1} h+x_{0}^{p} e^{(p)}+y_{0}^{p} f^{(p)}+z_{0}^{p} h^{(p)}+\sum_{\substack{i+j+\ell=p \\
i, j, \ell<p}} x_{0}^{i} y_{0}^{j} z_{0}^{\ell} e^{(i)} f^{(j)}\left(\begin{array}{c}
h \\
\ell
\end{array}\right) .
$$

Our motivational example for $G=\mathbb{G}_{a(1)} \times \mathbb{G}_{a(1)}$ from the beginning of this section is a special case of Example 2.6(1).

Example 2.7. Let $G=\mathbb{G}_{a(1)}^{\times r}$. Then $G$ corresponds to the abelian $p$-nilpotent Lie algebra $g_{a}^{\oplus r}$, and $k G=k\left[u_{0}, \ldots, u_{r-1}\right] /\left(u_{0}^{p}, \ldots, u_{r-1}^{p}\right)$. We have $V(G) \simeq \mathbb{A}^{r}$, and $k[V(G)] \simeq k\left[X_{0}, \ldots, X_{r-1}\right]$, where all generators are in degree one. Then $\Theta_{G} \in k G \otimes k[V(G)]$ is given by the simple formula

$$
\Theta_{G}=u_{0} X_{0}+\cdots+u_{r-1} X_{r-1} .
$$

It is useful to contrast this formula with the much more complicated result for $G=\mathbb{G}_{a(r)}$ in Example 2.6(2).

To complement Example 2.6. we make explicit the action of $\Theta_{G}$ on some representation of $G$ for each of the four types of finite group schemes we have been considering in examples.

Example 2.8. (1) Let $G=\mathfrak{g}$ and let $M=\mathfrak{g}^{\text {ad }}$ denote the adjoint representation of the $p$-restricted Lie algebra $\mathfrak{g}$; let $\left\{x_{i}\right\}$ be a basis for $\mathfrak{g}$. We identify $\Theta_{\underline{\mathfrak{g}}}$ as the $k\left[N_{p}(\mathfrak{g})\right]$-linear endomorphism

$$
\Theta_{\underline{\mathfrak{g}}}: \mathfrak{g}^{a d} \otimes k\left[N_{p}(\mathfrak{g})\right] \rightarrow \mathfrak{g}^{a d} \otimes k\left[N_{p}(\mathfrak{g})\right], \quad x \otimes 1 \mapsto \sum_{i}\left[x_{i}, x\right] \otimes X_{i},
$$

where $X_{i}$ is the image under the projection $S^{*}\left(\mathfrak{g}^{\#}\right) \rightarrow k\left[N_{p}(\mathfrak{g})\right]$ of the dual basis element to $x_{i}$.

(2) Let $M$ denote the cyclic $k \mathbb{G}_{a(r)}$-module

$$
M=k\left[u_{0}, \ldots, u_{r-1}\right] /\left(u_{0}, u_{1}^{p}, \ldots, u_{r-1}^{p}\right) \simeq k\left[u_{1}, \ldots, u_{r-1}\right] /\left(u_{1}^{p}, \ldots, u_{r-1}^{p}\right) .
$$

As recalled in Example [2.6 $(2), k\left[V\left(\mathbb{G}_{a(r)}\right)\right]=k\left[\mathbb{A}^{r}\right]=k\left[a_{0}, \ldots, a_{r-1}\right], k \mathbb{G}_{a(r)}=$ $k\left[u_{0}, \ldots, u_{r-1}\right] /\left(u_{i}^{p}\right)$, and

$$
\Theta_{\mathbb{G}_{a(r)}} \in A\left[u_{0}, \ldots, u_{r-1}\right] /\left(u_{i}^{p}\right)
$$

is given by the complicated, but explicit formula (2.6.3). We conclude that

$$
\Theta_{\mathbb{G}_{a(r)}}: M \otimes A \rightarrow M \otimes A
$$


is the $A$-linear endomorphism sending $u_{i}$ to $\bar{\Theta}_{\mathbb{G}_{a(r)}} \cdot u_{i}$, where $\bar{\Theta}_{\mathbb{G}_{a(r)}}$ is the image of $\Theta_{\mathbb{G}_{a(r)}}$ under the projection $A\left[u_{0}, \ldots, u_{r-1}\right] /\left(u_{i}^{p}\right) \rightarrow A\left[u_{1}, \ldots, u_{r-1}\right] /\left(u_{i}^{p}\right)$.

(3) Let $M$ be the restriction to $\mathrm{GL}_{n(r)}$ of the canonical $n$-dimensional rational $\mathrm{GL}_{n}$-module $V_{n}$. By Example 1.12(3), $A=k\left[V\left(\mathrm{GL}_{n(r)}\right]\right.$ is the quotient of $k\left[\mathfrak{g l}_{n}\right]^{\otimes r}$ by the ideal generated by the equations satisfied by an $r$-tuple of $n \times n$-matrices with the property that each matrix is $p$-nilpotent and that the matrices pairwise commute. The complexity of the map

$$
\Theta_{\mathrm{GL}_{n(r)}}: V_{n} \otimes A \rightarrow V_{n} \otimes A
$$

is revealed even in the case $n=2$, which is worked out explicitly below.

(4) Let $M$ be the restriction to $\mathrm{SL}_{2(2)}$ of the rational $\mathrm{GL}_{2}-$ representation $V_{2}$. Then Example 1.12(4) gives an explicit description of $A=k\left[V\left(\mathrm{SL}_{2(2)}\right)\right]$ as a quotient of $k\left[x_{0}, y_{0}, z_{0}, x_{1}, y_{1}, z_{1}\right]$ and (2.6.5) gives $\Theta_{\mathrm{SL}_{2(2)}}$ explicitly. Since $V_{2}$ is a homogeneous polynomial representation of $\mathrm{GL}_{2}$ of degree 1 , the divided powers $e^{(p)}, f^{(p)}$ and $h^{(p)}$ as well as all products of the form $e^{(i)} f^{(j)}\left(\begin{array}{l}h \\ \ell\end{array}\right)$ act trivially on $M$. Hence, the map

$$
\Theta_{\mathrm{SL}_{2(2)}}: M \otimes A \rightarrow M \otimes A
$$

is given by the matrix

$$
A^{2} \stackrel{\left[\begin{array}{cc}
z_{1} & x_{1} \\
y_{1} & -z_{1}
\end{array}\right]}{\longrightarrow} A^{2} .
$$

When viewing group schemes as functors, it is often convenient to think of $G_{k[V(G)]}$ as $G \times V(G)$ (i.e., $G \times V(G)=\operatorname{Spec}(k[V(G)] \otimes k[G])$ ). From this point of view, $\mathcal{U}_{G}$ has the form

$$
\mathcal{U}_{G}: \mathbb{G}_{a(r)} \times V(G) \longrightarrow G \times V(G) .
$$

The following naturality property of $\Theta_{G}$ will prove useful when we consider $M \otimes k[V(G)]$ as a free, coherent sheaf on $V(G)$ and restrict this sheaf to $V(H) \subset$ $V(G)$ equipped with its action of $H$.

Proposition 2.9. Choose $r>0$ and consider a closed embedding $i: H \hookrightarrow G$ of algebraic affine group schemes over $k$. Let $\phi: V_{r}(H) \rightarrow V_{r}(G)$ be the closed embedding of affine schemes induced by $i$, with an associated surjective map $\phi^{*}: k\left[V_{r}(G)\right]$ $\rightarrow k\left[V_{r}(H)\right]$ on coordinate algebras. Then the following square commutes:

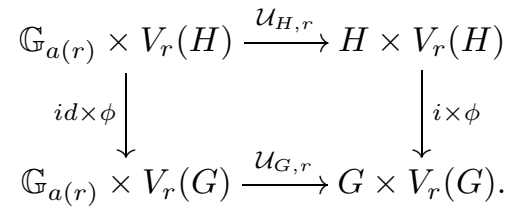

Consequently, the following square of $k$-linear maps commutes:

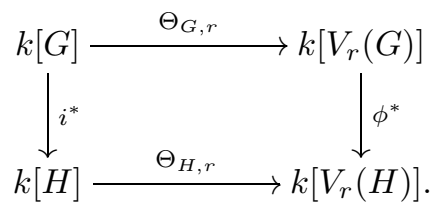


Thus, for any rational $G$-module $M$ we have a compatibility of coactions on $M$ :

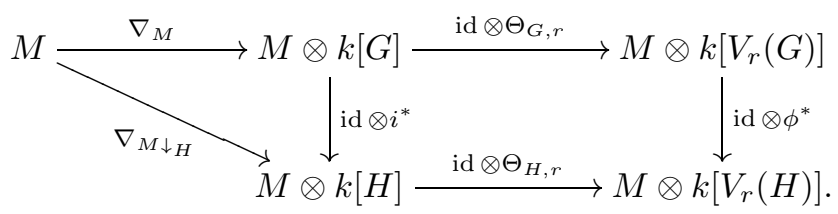

Proof. The fact that $\phi: V_{r}(H) \rightarrow V_{r}(G)$ induced by the closed embedding $i: H \hookrightarrow$ $G$ is itself a closed embedding is given by [25, 1.5]. By universality of $\mathcal{U}_{G, r}$, the composition $(i \times i d) \circ \mathcal{U}_{H, r}: \mathbb{G}_{a(r)} \times V_{r}(H) \rightarrow G \times V_{r}(H)$ is obtained by pull-back of $\mathcal{U}_{G, r}$ via some morphism $V_{r}(H) \rightarrow V_{r}(G)$. By comparing maps on $R$-valued points, we verify that this morphism must be $\phi$. This implies the commutativity of (2.9.1).

The commutative square (2.9.1) gives a commutative square on coordinate algebras:

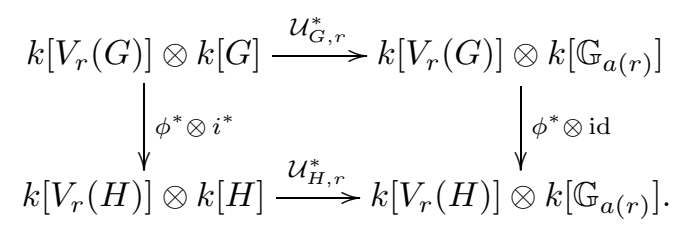

Concatenating (2.9.4) on the right with the commutative square of linear maps

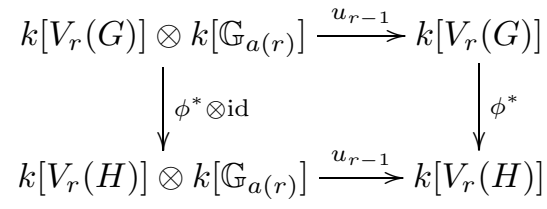

and with the inclusions $k[G] \rightarrow k\left[V_{r}(G)\right] \otimes k[G]$ and $k[H] \rightarrow k\left[V_{r}(H)\right] \otimes k[H]$ on the left, we obtain a commutative diagram:

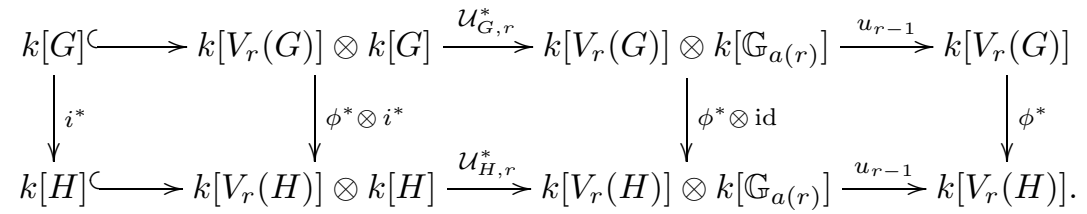

Eliminating the middle square, we obtain the square (2.9.2). Hence, (2.9.2) is commutative.

Finally, the commutativity of (2.9.3) follows immediately from the commutativity of (2.9.2).

Pre-composition determines a natural action $V_{r}(G) \times V_{r}\left(\mathbb{G}_{a(r)}\right) \rightarrow V_{r}(G)$ for any algebraic affine group scheme $G$. Recall from [25, 1.10] that $V_{r}\left(\mathbb{G}_{a(r)}\right) \simeq \mathbb{A}^{r}$ : morphisms of group schemes $\mathbb{G}_{a(r), A} \rightarrow \mathbb{G}_{a(r), A}$ over $A$ have associated maps on coordinate algebras $A[T] / T^{p^{r}} \rightarrow A[T] / T^{p^{r}}$ given by additive polynomials, that is, polynomials of the form $a_{0} T+a_{1} T^{p}+\cdots+a_{r-1} T^{p^{r-1}}$. Restricting the action $V_{r}(G) \times V_{r}\left(\mathbb{G}_{a(r)}\right) \rightarrow V_{r}(G)$ to linear polynomials $\mathbb{A}^{1} \subset V_{r}\left(\mathbb{G}_{a(r)}\right) \simeq \mathbb{A}^{r}$ determines a natural action

$$
V_{r}(G) \times \mathbb{A}^{1} \rightarrow V_{r}(G),
$$

which is equivalent by [25, 1.11] to a functorial grading on $k\left[V_{r}(G)\right]$. 
Proposition 2.10. Let $G$ be an algebraic affine group scheme over $k$. Then the coordinate algebra $k\left[V_{r}(G)\right]$ of $V_{r}(G)$ is a graded algebra generated by homogeneous generators of degrees $p^{i}, 0 \leq i<r$.

Proof. The coordinate algebra $k\left[V_{r}(G)\right]$ is graded by [25, 1.12]. If $G=\mathrm{GL}_{N}$, then an $R$-valued point of $V_{r}\left(\mathrm{GL}_{N}\right)$ is given by an $r$-tuple of $N \times N$ pairwise commuting, $p$-nilpotent matrices with entries in $R,\left(\alpha_{0}, \ldots, \alpha_{r-1}\right)$. The action of $c \in V\left(\mathbb{G}_{a(1)}\right)(R)$ on $\left(\alpha_{0}, \alpha_{1}, \ldots, \alpha_{r-1}\right) \in V\left(\mathrm{GL}_{N(r)}\right)(R)$ is given by the formula

$$
\left(\alpha_{0}, \alpha_{1}, \ldots, \alpha_{r-1}\right) \times c=\left(c \alpha_{0}, c^{p} \alpha_{1}, \ldots, c^{p^{r-1}} \alpha_{r-1}\right) .
$$

Hence, the coordinate functions of the matrix $\alpha_{i}$ have grading $p^{i}$, and, therefore, $k\left[V_{r}\left(G L_{N}\right)\right]$ is generated by homogeneous elements of degree $p^{i}, 0 \leq i<r$.

Let $i: G \rightarrow G L_{N}$ be a closed embedding of a finite group scheme $G$ into some $\mathrm{GL}_{N}$. The naturality of the grading (see [25, 1.12]) implies that the surjective map $\phi: k\left[V_{r}\left(\mathrm{GL}_{N}\right)\right] \rightarrow k\left[V_{r}(G)\right]$ is a map of graded algebras.

Proposition 2.11. For any algebraic affine group scheme $G$ and integer $r>0$, the $k$-linear map

$$
\Theta_{G, r}: k[G] \rightarrow k\left[V_{r}(G)\right]
$$

has image contained in the homogeneous summand of $k\left[V_{r}(G)\right]$ of degree $p^{r-1}$.

If $G$ is infinitesimal, then this is equivalent to the following:

$$
\mathcal{U}_{G, r *}: k \mathbb{G}_{a(r)} \otimes k\left[V_{r}(G)\right] \rightarrow k G \otimes k\left[V_{r}(G)\right]
$$

sends $u_{r-1} \otimes 1 \in k \mathbb{G}_{a(r)} \otimes k\left[V_{r}(G)\right]$ to some $\sum x_{i} \otimes a_{i} \in k G \otimes k\left[V_{r}(G)\right]$ with each $a_{i} \in k\left[V_{r}(G)\right]$ homogeneous of degree $p^{r-1}$.

Proof. Let $A=k\left[V_{r}(G)\right]$. Since $\Theta_{G, r}$ factors through the $r^{\text {th }}$ Frobenius kernel of $G$, we may assume $G$ is infinitesimal. Let $\left\langle\lambda_{i}\right\rangle$ be a set of linear generators of $k[G]$ and $\left\langle\check{\lambda}_{i}\right\rangle$ be the dual set of linear generators of $k G$. Then $\mathcal{U}_{G, r, *}\left(u_{r-1}\right)=\sum \check{\lambda}_{i} \otimes f_{i}$ if and only if $u_{r-1}\left(\mathcal{U}_{G}^{*}\left(\lambda_{i}\right)\right)=f_{i}$ if and only if $\mathcal{U}_{G, r}^{*}\left(\lambda_{i}\right)=\cdots+f_{i} T^{p^{r-1}}+\cdots$. Hence, the assertion that $\Theta_{G, r}$ is homogeneous of degree $p^{r-1}$ is equivalent to showing that the map $k[G] \rightarrow A$ defined by reading the coefficient of

$$
\mathcal{U}_{G, r}^{*}: k[G] \rightarrow A \otimes k[G] \rightarrow A \otimes k\left[\mathbb{G}_{a(r)}\right] \rightarrow A[T] / T^{p^{r}}
$$

of the monomial $T^{p^{r-1}}$ is homogeneous of degree $p^{r-1}$.

The coordinate algebra $k\left[\mathbb{G}_{a(r)}\right] \simeq k[T] / T^{p^{r}}$ has a natural grading with $T$ assigned degree 1. This grading corresponds to the monoidal action of $\mathbb{A}^{1}$ on $\mathbb{G}_{a(r)}$ by multiplication:

$$
\mathbb{G}_{a(r)} \times \mathbb{A}^{1} \stackrel{s \times a \mapsto s a}{\longrightarrow} \mathbb{G}_{a(r)} .
$$

We proceed to prove that this action is compatible with the action of $\mathbb{A}^{1}$ on $V_{r}(G)$ which defines the grading on $A$ in the sense that the following diagram commutes:

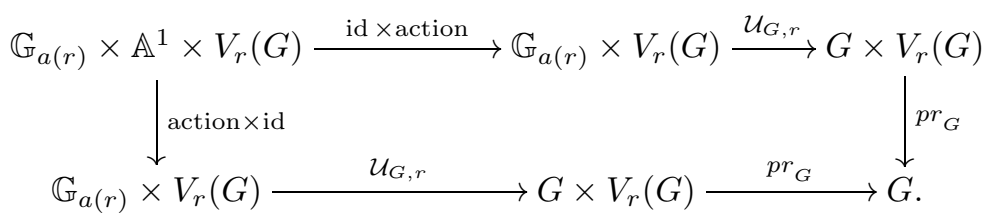


Commutativity of (2.11.1) is equivalent to the commutativity of the corresponding diagram of $S$-valued points for any choice of finitely generated commutative $k$-algebras $S$ and element $a \in S$ :

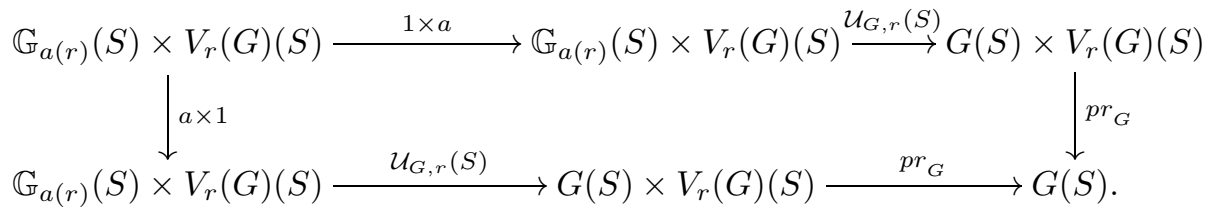

Choose an embedding of $G$ into some $\mathrm{GL}_{N(r)}$. Using Proposition 2.9 and the naturality with respect to change of $G$ of the action of $\mathbb{A}^{1}$ on $V_{r}(G)$, we can compare the diagram (2.11.2) for $G$ and for $\mathrm{GL}_{N(r)}$. The injectivity of $G(S) \rightarrow$ $\operatorname{GL}_{N(r)}(S)$ implies that it suffices to assume that $G=\operatorname{GL}_{N(r)}$. Let $s \in \mathbb{G}_{a(r)}(S)$, $\underline{\alpha}=\left(\alpha_{0}, \ldots, \alpha_{r-1}\right) \in V_{r}\left(\mathrm{GL}_{N}\right)(S)$. Then $a \circ \underline{\alpha}=\left(a \alpha_{0}, a^{p} \alpha_{1}, \ldots, a^{p^{r-1}} \alpha_{r-1}\right)$, so $\exp _{\alpha}(s)=\exp \left(s \alpha_{0}\right) \exp \left(s^{p} \alpha_{1}\right) \cdots \exp \left(s^{p^{r-1}} \alpha_{p-1}\right) \in \mathrm{GL}_{N(r)}(S)$ by (1.10.1). Thus, restricted to the point $(s, \underline{\alpha}) \in\left(\mathbb{G}_{a(r)} \times V_{r}\left(\mathrm{GL}_{N}\right)\right)(S)$, (2.11.2) becomes

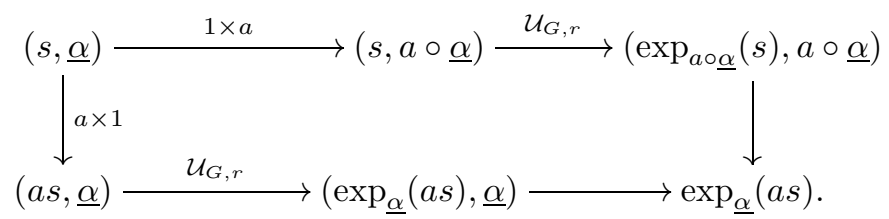

Commutativity of (2.11.3) is implied by the evident equality $\exp _{a \circ \underline{\alpha}}(s)=\exp _{\underline{\alpha}}(a s)$.

Consequently, we have a commutative diagram on coordinate algebras corresponding to (2.11.1):

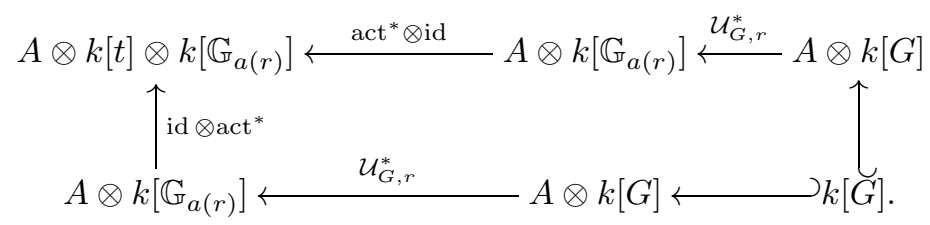

The map act* $: A \longrightarrow A \otimes k[t]=A \otimes k\left[\mathbb{A}^{1}\right]$ of the upper horizontal arrow is the map on coordinate algebras which corresponds to the grading on $A$. The left vertical map corresponds to the grading on $k\left[\mathbb{G}_{a(r)}\right] \simeq k[T] / T^{p^{r}}$ and is given explicitly by $T \mapsto t \otimes T$.

For $\lambda \in k[G]$, write $\mathcal{U}_{G, r}^{*}(1 \otimes \lambda)=\sum f_{i} \otimes c_{i} T^{i} \in A \otimes k\left[\mathbb{G}_{a(r)}\right]$. The composition of the lower horizontal and left vertical maps of (2.11.4 sends $\lambda$ to $\sum f_{i} \otimes t^{i} \otimes c_{i} T^{i}$. On the other hand, the composition of the right vertical and upper horizontal maps of (2.11.4 sends $\lambda$ to $\sum \operatorname{act}^{*}\left(f_{i}\right) \otimes c_{i} T^{i}$. We conclude that

$$
f_{i} \otimes t^{i}=\operatorname{act}^{*}\left(f_{i}\right)
$$

so that $f_{i}$ is homogeneous of degree $i$.

As a corollary (of the proof) of Proposition 2.11, we see why for $G$ infinitesimal of height $\leq r$ the homogeneous degree of $\Theta_{G, r} \in k G \otimes k\left[V_{r}(G)\right]$ is $p^{r-1}$, whereas the homogeneous degree of $\Theta_{G, r+1} \in k G \otimes k\left[V_{r+1}(G)\right]$ is $p^{r}$. 
Corollary 2.12. Let $G$ be an infinitesimal group of height $\leq r$. Then the map $i^{*}: k\left[V_{r+1}(G)\right] \rightarrow k\left[V_{r}(G)\right]$ of Proposition 2.2 is a graded isomorphism which divides degrees by $p$.

Proof. Let $\pi^{*}: k\left[V_{r}(G)\right] \rightarrow k\left[V_{r+1}(G)\right]$ be the inverse of $i^{*}$. The commutativity of (2.11.1) implies that we may compute the effect on degree of $\pi^{*}$ by identifying the effect on degree of the map $p^{*}: k\left[\mathbb{G}_{a(r)}\right]=k[t] / t^{p^{r}} \rightarrow k[t] / t^{p^{r+1}}=k\left[\mathbb{G}_{a(r+1)}\right]$. Yet this map clearly multiplies degree by $p$.

\section{LOCAL JORDAN TYPE}

The purpose of this section is to exploit our universal $p$-nilpotent operator $\Theta_{G}$ to investigate the local Jordan type of a finite dimensional $k G$-module $M$. The local Jordan type of $M$ gives much more detailed information about a $k G$-module $M$ than the information which can be obtained from the support variety (or, rank variety) of $M$. In this section, we work through various examples, give an algorithm for computing local Jordan types, and understand the effect of Frobenius twists. Moreover, we establish restrictions on the rank and dimension of $k G$-modules of constant Jordan type.

Definition 3.1. Let $G$ be an infinitesimal group scheme and $v \in V(G)$. Let $k(v)$ denote the residue field of $V(G)$ at $v$, and let

$$
\mu_{v}=\mathcal{U}_{G} \otimes_{k[V(G)]} k(v): \mathbb{G}_{a(r), k(v)} \rightarrow G_{k(v)}
$$

be the associated 1-parameter subgroup (for $r \geq \mathrm{ht}(\mathrm{G})$ ). We define the local $p$ nilpotent operator at $v, \theta_{v}$, to be

$$
\theta_{v}=\Theta_{G} \otimes_{k[V(G)]} k(v)=\mu_{v *}\left(u_{r-1}\right) \in k(v) G .
$$

Equivalently, for a $k(v)$-rational point $v: \operatorname{Spec} k(v) \rightarrow V(G)$, we define $\theta_{v}=\Theta_{G}(v)$, the evaluation of $\Theta_{G}$ at $v$ :

$$
\theta_{v}: k[G] \stackrel{\Theta_{G}}{\longrightarrow} k[V(G)] \longrightarrow k(v),
$$

where the second map corresponds to the point $v$.

In the special case that $G=\mathrm{GL}_{n(r)}$ for some $n>0$, we use the alternate notation $\theta_{\underline{\alpha}}$ for the local $p$-nilpotent operator at $\underline{\alpha}=\left(\alpha_{0}, \ldots, \alpha_{r-1}\right) \in V\left(\mathrm{GL}_{n(r)}\right) \simeq$ $N_{p}^{[r]}\left(g l_{n}\right)$ :

$$
\theta_{\underline{\alpha}}=\exp _{\underline{\alpha}, *}\left(u_{r-1}\right) \in k(\underline{\alpha}) \mathrm{GL}_{n(r)},
$$

where $k(\underline{\alpha})$ is the residue field of $\underline{\alpha} \in V\left(\mathrm{GL}_{n(r)}\right)$.

Let $K$ be a field. Then a finite dimensional $K[u] / u^{p}$-module $M$ is a direct sum of cyclic modules of dimension ranging from 1 to $p$. We may thus write $M \simeq a_{p}[p]+\cdots+a_{1}[1]$, where $[i]$ is the cyclic $K[u] / u^{p}$-module $K[u] / u^{i}$ of dimension $i$. We refer to the $p$-tuple

$$
\operatorname{JType}(M, u)=\left(a_{p}, \ldots, a_{1}\right)
$$

as the Jordan type of the $K[u] / u^{p}$-module $M$. We also refer to $\operatorname{JType}(M, u)$ as the Jordan type of the $p$-nilpotent operator $u$ on $M$.

For simplicity, we introduce the following notation. 
Definition 3.2. With notation as in Definition 3.1 we set

$$
\operatorname{JType}\left(M, \theta_{v}\right) \equiv \operatorname{JType}\left(\left(\mu_{v, *}\right)^{*}\left(M_{k(v)}\right), u_{r-1}\right) \text {. }
$$

We refer to this Jordan type as the local Jordan type of $M$ at $v \in V(G)$.

Remark 3.3. Essentially by definition, the rank variety $V(G)_{M}$ of a finite dimensional $k G$-module for an infinitesimal group scheme $G$ is the closed, reduced subscheme of $V(G)$ consisting of those points $v \in V(G)$ at which the local Jordan type of $M$ has some Jordan block of size $<p$. In other words, those $v \in V(G)$ for which $\operatorname{JType}\left(M, \theta_{v}\right) \neq \frac{\operatorname{dim} M}{p}[p]$.

The following proposition will enable us to make more concrete and explicit the local Jordan type of a $k G$-module $M$ at a given 1-parameter subgroup of $G$.

Proposition 3.4. Let $\underline{\alpha}=\left(\alpha_{0}, \ldots, \alpha_{r-1}\right) \in V\left(\mathrm{GL}_{n(r)}\right)$ be an $r$-tuple of p-nilpotent pairwise commuting matrices. Let $M$ be a $k \mathrm{GL}_{(r)}$-module of dimension $N$, and let $\rho: \mathrm{GL}_{m(r)} \rightarrow \mathrm{GL}_{N}$ be the associated structure map. The $(i, j)$-matrix entry of the action of the local p-nilpotent operator $\theta_{\alpha} \in k(\underline{\alpha}) \mathrm{GL}_{n(r)}$ of (3.1.1) on $M$ equals the coefficient of $T^{p^{r-1}}$ of

$$
\left(\exp _{\underline{\alpha}}\right)^{*}\left(\rho^{*} X_{i, j}\right) \in k(\underline{\alpha})\left[\mathbb{G}_{a(r)}\right] \simeq k(\underline{\alpha})[T] / T^{p^{r}},
$$

where $\left\{X_{i, j}, 1 \leq i, j \leq N\right\}$ are the matrix coordinate functions of $\mathrm{GL}_{N}$.

Proof. Let $\left\langle m_{i}\right\rangle_{1 \leq i \leq N}$ be the basis of $M$ corresponding to the structure map $\rho$. The structure of $M$ as a comodule for $k\left[\mathrm{GL}_{n(r)}\right]$ is given by

$$
M \rightarrow M \otimes k\left[\mathrm{GL}_{n(r)}\right], \quad m_{j} \mapsto \sum_{i} m_{i} \otimes \rho^{*} X_{i, j},
$$

and thus the comodule structure of $M_{k(\underline{\alpha})}$ for $k(\underline{\alpha})\left[\mathbb{G}_{a(r)}\right]$ is given by

$$
M \rightarrow M \otimes k(\underline{\alpha})\left[\mathbb{G}_{a(r)}\right], \quad m_{j} \mapsto \sum_{i} m_{i} \otimes \exp _{\underline{\alpha}}^{*}\left(\rho^{*} X_{i, j}\right) .
$$

The proposition follows from the fact that $u_{r-1}: k(\underline{\alpha})\left[\mathbb{G}_{a(r)}\right] \rightarrow k(\underline{\alpha})$ is given by reading the coefficient of $T^{p^{r-1}} \in k(\underline{\alpha})\left[\mathbb{G}_{a(r)}\right]$.

Example 3.5. We investigate the local Jordan type of the various representations considered in Example 2.8.

(1) Consider the adjoint representation $M=\mathfrak{g}^{\text {ad }}$ of a $p$-restricted Lie algebra $\mathfrak{g}$ and a 1-parameter subgroup

$$
\mu_{X}: \mathbb{G}_{a(1), K} \rightarrow \underline{\mathfrak{g}}_{K}, \quad \text { inducing } K[u] / u^{p} \rightarrow \mathfrak{u}\left(\mathfrak{g}_{K}\right)
$$

sending $u$ to some $p$-nilpotent $X \in \mathfrak{g}_{K}$. The local Jordan type of $\mathfrak{g}^{\text {ad }}$ at $\mu_{X}$ is simply the Jordan type of the endomorphism $\operatorname{ad}_{X}: \mathfrak{g}_{K}^{a d} \rightarrow \mathfrak{g}_{K}^{\text {ad }}$,

$$
\operatorname{JType}\left(\mathfrak{g}^{\text {ad }}, \theta_{X}\right)=\operatorname{JType}(X) .
$$

(2) Let $M=k\left[\mathbb{G}_{a(r)}\right] /\left(u_{0}\right) \simeq k\left[u_{1}, \ldots, u_{r-1}\right] /\left(u_{1}^{p}, \ldots, u_{p-1}^{p}\right)$ be a cyclic $k \mathbb{G}_{a(r)}=$ $k\left[u_{0}, \ldots, u_{r-1}\right] /\left(u_{0}^{p}, \ldots, u_{r-1}^{p}\right)$-module, and let $\mu_{\underline{a}}: \mathbb{G}_{a(r)} \rightarrow \mathbb{G}_{a(r)}$ be a 1-parameter subgroup for some $\underline{a}$ of $V\left(\mathbb{G}_{a(r)}\right)=\mathbb{A}^{r}$. Then

$$
\operatorname{JType}\left(M, \theta_{\underline{a}}\right)= \begin{cases}p^{r-2}[p], & \exists i>0, a_{i} \neq 0, \\ p^{r-1}[1], & \text { otherwise. }\end{cases}
$$


(3) Let $G=\mathrm{GL}_{n(r)}$, and let $V_{n}$ be the canonical $n$-dimensional rational representation of $\mathrm{GL}_{n(r)}$. We apply Proposition 3.4, observing that $\rho$ for $V_{n}$ is simply the natural inclusion $\mathrm{GL}_{n(r)} \subset \mathrm{GL}_{n}$. Since

$$
\exp _{\underline{\alpha}}^{*}\left(X_{i, j}\right)=\sum_{\ell=0}^{p^{r}-1}\left[\beta_{\ell}\right]_{i, j} t^{\ell},
$$

where $\beta_{\ell}$ are matrices determined by $\alpha_{i}$ as in Proposition 1.7, we conclude that

$$
\operatorname{JType}\left(V_{n}, \theta_{\underline{\alpha}}\right)=\operatorname{JType}\left(\alpha_{r-1}\right) .
$$

Specializing to $r=2$,

$$
\operatorname{JType}\left(V_{n}, \theta_{\left(\alpha_{0}, \alpha_{1}\right)}\right)=\alpha_{1} .
$$

(4) "Specializing" to $G=\mathrm{SL}_{2(2)}$, consider $\underline{\alpha}=\left(\left[\begin{array}{cc}c_{0} & a_{0} \\ b_{0} & -c_{0}\end{array}\right],\left[\begin{array}{cc}c_{1} & a_{1} \\ b_{1} & -c_{1}\end{array}\right]\right)$. Then JType $\left(V_{2}, \theta_{\underline{\alpha}}\right)$ equals the Jordan type of the matrix $\left[\begin{array}{cc}c_{1} & a_{1} \\ b_{1} & -c_{1}\end{array}\right]$.

We extend Example $3.5(3)$ by considering tensor powers $V_{n}^{\otimes d}$ of the canonical rational representation of $\mathrm{GL}_{n}$ restricted to $\mathrm{GL}_{n(2)}$. In this example, the role of both entries of the pair $\underline{\alpha}=\left(\alpha_{0}, \alpha_{1}\right)$ is non-trivial.

Example 3.6. Consider the $N=n^{d}$-dimensional rational $\mathrm{GL}_{n}$-module $M=V_{n}^{\otimes d}$ where $V_{n}$ is the canonical $n$-dimensional rational $\mathrm{GL}_{n}$-module. Let $\rho: \mathrm{GL}_{n(r)} \rightarrow$ $\mathrm{GL}_{N}$ be the representation of $M$ restricted to $\mathrm{GL}_{n(r)}$. A basis of $M$ is $\left\{e_{i_{1}} \otimes \cdots \otimes\right.$ $\left.e_{i_{d}} ; 1 \leq i_{j} \leq n\right\}$, where $\left\{e_{i_{j}} ; 1 \leq i_{j} \leq n\right\}$ is a basis for $V_{n}$ for each $j, 1 \leq j \leq d$. Let $\left\{X_{i_{1}, j_{1} ; \ldots ; i_{d}, j_{d}}, 1 \leq i_{t}, j_{t} \leq n\right\}$ denote the matrix coordinate functions on $\mathrm{GL}_{N}$, and let $\left\{Y_{s, t}, 1 \leq s, t \leq n\right\}$ denote the matrix coordinate functions of $\mathrm{GL}_{n}$.

Then $\rho^{*}: k\left[\mathrm{GL}_{N}\right] \rightarrow k\left[\mathrm{GL}_{n(r)}\right]$ is given by

$$
X_{i_{1}, j_{1} ; \ldots ; i_{d}, j_{d}} \mapsto Y_{i_{1}, j_{1}} \cdots Y_{i_{d}, j_{d}} .
$$

Thus,

$$
\left(\exp _{\underline{\alpha}}\right)^{*}\left(\rho^{*}\left(X_{i_{1}, j_{1} ; \ldots ; i_{d}, j_{d}}\right)\right)=\left(\exp _{\underline{\alpha}}\right)^{*}\left(Y_{i_{1}, j_{1}}\right) \cdots\left(\exp _{\underline{\alpha}}\right)^{*}\left(Y_{i_{d}, j_{d}}\right) .
$$

Now, specialize to $r=2$ so that we can make this more explicit. Then the coefficient of $T^{p}$ of $\left(\exp _{\left(\alpha_{0}, \alpha_{1}\right)}\right)^{*}\left(\rho^{*}\left(X_{i_{1}, j_{1} ; \ldots ; i_{d}, j_{d}}\right)\right)$ is

$$
\sum_{k=1}^{d}\left(\alpha_{1}\right)_{i_{k}, j_{k}}+\sum_{\substack{0 \leq f_{k}<p \\ f_{1}+\cdots+f_{d}=p}} \frac{1}{f_{1} !} \cdots \frac{1}{f_{d} !}\left(\left(\alpha_{0}\right)^{f_{1}}\right)_{i_{1}, j_{1}} \cdots\left(\left(\alpha_{0}\right)^{f_{d}}\right)_{i_{d}, j_{d}} .
$$

This gives the action of $\theta_{\left(\alpha_{0}, \alpha_{1}\right)}$ on $M$.

To simplify matters even further, consider the special case $\left(\alpha_{0}\right)^{2}=0$. For $1 \leq$ $d<p, \theta_{\left(\alpha_{0}, \alpha_{1}\right)}$ on $M$ is given by the $N \times N$-matrix

$$
\left(i_{1}, j_{1} ; \ldots ; i_{d}, j_{d}\right) \mapsto\left(\sum_{k=1}^{d}\left(\alpha_{1}\right)_{i_{k}, j_{k}}\right) .
$$

For $d=p$, the action of $\theta_{\left(\alpha_{0}, \alpha_{1}\right)}$ on $M$ is given by the $N \times N$-matrix

$$
\left(i_{1}, j_{1} ; \ldots ; i_{p}, j_{p}\right) \mapsto\left(\sum_{k=1}^{p}\left(\alpha_{1}\right)_{i_{k}, j_{k}}+\left(\alpha_{0}\right)_{i_{1}, j_{1}} \cdots\left(\alpha_{0}\right)_{i_{p}, j_{p}}\right) .
$$


An analogous calculation applies to the $d$-fold symmetric product $S^{d}\left(V_{n}\right)$ and $d$-fold exterior product $\Lambda^{d}\left(V_{n}\right)$ of the canonical $n$-dimensional rational $\mathrm{GL}_{n}$-module $V_{n}$.

The proof of Proposition 3.4 applies equally well to prove the following straightforward generalization, which one may view as an algorithmic method of computing the "local Jordan type" of a $k G$-module $M$ of dimension $N$. The required input is an explicit description of the map on coordinate algebras $\rho^{*}$ given by $\rho: G \rightarrow \mathrm{GL}_{N}$ determining the $k G$-module $M$.

Theorem 3.7. Let $G$ be an infinitesimal group scheme of height $\leq r$, and let $\rho: G \rightarrow \mathrm{GL}_{N}$ be a representation of $G$ on a vector space $M$ of dimension $N$. Consider some $v \in V(G)$, and let $\mu_{v}: \mathbb{G}_{a(r), k(v)} \rightarrow G_{k(v)}$ be the corresponding 1-parameter subgroup of height $r$. Then the $(i, j)$-matrix entry of the action of $\theta_{v} \in k(v) G$ on $M$ equals the coefficient of $T^{p^{r-1}}$ of

$$
\left(\mu_{v}\right)^{*}\left(\rho^{*} X_{i, j}\right) \in k(v)\left[\mathbb{G}_{a(r)}\right],
$$

where $\left\{X_{i, j}, 1 \leq i, j \leq N\right\}$ are the matrix coordinate functions of $\mathrm{GL}_{N}$.

As a simple corollary of Theorem 3.7 we give a criterion for the local Jordan type of the $k G$-module $M$ to be trivial (i.e., equal to $(\operatorname{dim} M)[1])$ at a 1-parameter subgroup $\mu_{v}, v \in V(G)$.

Corollary 3.8. With the hypotheses and notation of Theorem 3.7 ,

$$
\operatorname{JType}\left(M, \theta_{v}\right)=\operatorname{JType}\left(\left(\mu_{v, *}\right)^{*}\left(M_{k(v)}\right), u_{r-1}\right)=(\operatorname{dim} M)[1]
$$

if $\operatorname{deg}\left(\rho \circ \mu_{v}\right)^{*}\left(X_{i, j}\right)<p^{r-1}$ for all $1 \leq i, j \leq N$.

One means of constructing $k G$-modules is by applying Frobenius twists to known $k G$-modules. Our next objective is to establish (in Proposition 3.10) a simple relationship between the $p$-nilpotent operator $\theta_{\underline{\alpha}}$ on a $k \mathrm{GL}_{n(r)}$-module $M$ and $\theta_{\underline{\alpha}}$ on the $s^{\text {th }}$ Frobenius twist $M^{(s)}$ of $M$ for any $0 \neq v \in V\left(\mathrm{GL}_{n(r)}\right)$.

Before formulating this relationship, we make explicit the definition of the Frobenius map for an arbitrary affine group scheme over $k$. Let $G$ be an affine group scheme over $k$ and define for any $s>0$ the $s^{\text {th }}$ Frobenius map $F^{s}: G \rightarrow G^{(s)}$ given by the $k$-linear algebra homomorphism

$$
F^{s *}: k\left[G^{(s)}\right]=k \otimes_{p^{s}} k[G] \rightarrow k[G], \quad a \otimes f \mapsto a \cdot f^{p^{s}},
$$

where $k \otimes_{p^{s}} k[G]$ is the base change of $k[G]$ along the $p^{s}$-power map $k \rightarrow k$ (an isomorphism only for $k$ perfect). If $G$ is defined over $\mathbb{F}_{p^{s}}$ (for example, if $G=\mathrm{GL}_{n}$ ), then we have a natural isomorphism

$$
k[G]=k \otimes p^{s} \mathbb{F}_{p^{s}}[G] \stackrel{\sim}{\longrightarrow} k \otimes_{p^{s}} k \otimes p^{s} \mathbb{F}_{p^{s}}[G]=k\left[G^{(s)}\right]
$$

so that $F^{s}$ can be viewed as a self-map of $G$.

Definition 3.9. If $M$ is a $k[G]$-comodule, then the $s^{\text {th }}$ Frobenius twist $M^{(s)}$ of $M$ is the $k$-vector space $k \otimes_{p^{s}} M$ equipped with the comodule structure

$$
F^{s *} \circ\left(k \otimes_{p^{s}} \nabla_{M}\right): M^{(s)} \rightarrow M^{(s)} \otimes k\left[G^{(s)}\right] \rightarrow M^{(s)} \otimes k[G] .
$$

If $G$ is a finite group scheme, then we shall view $M^{(s)}$ as a $k G$-module via the map $F_{*}^{s}: k G \rightarrow k G^{(s)}$ dual to (3.8.1). 
To be more explicit, suppose the $N$-dimensional $k G$-module $M$ is given by $\rho$ : $G \rightarrow \mathrm{GL}_{N}$ (so that $M=\rho^{*}\left(V_{N}\right)$, where $V_{N}$ is the canonical $N$-dimensional $\mathrm{GL}_{N^{-}}$ module) and assume that $G$ is defined over $\mathbb{F}_{p^{s}}$. Let $\mu_{v}: \mathbb{G}_{a(r), K} \rightarrow G_{K}$ be a 1-parameter subgroup, corresponding to some $v \in V(G)$. Then the identification of $M^{(s)}$ with $\left(\rho \circ F^{s}\right)^{*}\left(V_{N}\right)$ implies that

$$
\operatorname{JType}\left(M^{(s)}, \theta_{v}\right)=\operatorname{JType}\left(M, \theta_{F^{s}(v)}\right) \text {, }
$$

where $\theta_{F^{s}(v)}=\left(F^{s} \circ \mu_{v}\right)_{*}\left(u_{r-1}\right)$.

Let $G=\mathrm{GL}_{n(r)}$, and let $R$ be a finitely generated commutative $k$-algebra. The Frobenius self-map is given explicitly on the $R$-values of $\mathrm{GL}_{n(r)}$ by the formula

$$
F: \alpha \mapsto \phi(\alpha),
$$

where $\phi$ applied to $\alpha \in M_{n}(R)$ raises each entry of $\alpha$ to the $p^{\text {th }}$ power. For $t$ in $\mathbb{G}_{a(r)}(R)$, we compute

$$
\begin{gathered}
\left(F \circ \exp _{\left(\alpha_{0}, \ldots, \alpha_{r-1}\right)}\right)(t)=F\left(\exp \left(t \alpha_{0}\right) \exp \left(t^{p} \alpha_{1}\right) \cdots \exp \left(t^{p^{r-1}} \alpha_{r-1}\right)\right) \\
=\exp \left(t^{p} \phi\left(\alpha_{0}\right)\right) \exp \left(t^{p^{2}} \phi\left(\alpha_{1}\right)\right) \cdots \exp \left(t^{p^{r-1}} \phi\left(\alpha_{r-2}\right)\right)=\exp _{\left(0, \phi\left(\alpha_{0}\right), \ldots, \phi\left(\alpha_{r-2}\right)\right)}(t) .
\end{gathered}
$$

Iterating $s$ times, we obtain the following formula for $G=\mathrm{GL}_{n(r)}$ :

$$
F^{s} \circ \exp _{\left(\alpha_{0}, \ldots, \alpha_{r-1}\right)}=\exp _{\left(0,0, \ldots, 0, \phi^{s}\left(\alpha_{0}\right), \ldots, \phi^{s}\left(\alpha_{r-1-s}\right)\right)},
$$

where the first non-zero entry on the right happens at the $(s+1)^{\text {st }}$ place.

For $G=\mathbb{G}_{a(r)}$, the Frobenius map $F: \mathbb{G}_{a(r)} \rightarrow \mathbb{G}_{a(r)}$ is given by raising an element $a \in \mathbb{G}_{a(r)}(R)$ to the $p^{\text {th }}$ power. Let $\underline{a}=\left(a_{0}, \ldots, a_{r-1}\right)$ be a point in $V\left(\mathbb{G}_{a(r)}\right) \simeq \mathbb{A}^{r}$, and let $\mu_{\underline{a}}: \mathbb{G}_{a(r)} \rightarrow \mathbb{G}_{a(r)}$ be the corresponding 1-parameter subgroup. For $t \in \mathbb{G}_{a(r)}(R)$, we have $\mu(t)=a_{0}+a_{1} t+\cdots+a_{r-1} t^{p^{r-1}}$ (see [25, §1]). The following formula is now immediate:

$$
F^{s} \circ \mu_{\left(a_{0}, \ldots, a_{r-1}\right)}=\mu_{\left(0, \ldots, 0, a_{0}^{p^{s}}, \ldots, a_{r-1-s}^{p^{s}}\right)} .
$$

Combining (3.9.1) and (3.9.2), we derive the following proposition.

Proposition 3.10. Let $M$ be a finite dimensional representation of $\mathrm{GL}_{n(r)}$ and let $\underline{\alpha}=\left(\alpha_{0}, \ldots, \alpha_{r-1}\right)$ be a point in $V\left(\mathrm{GL}_{n(r)}\right)$. Then

$$
\operatorname{JType}\left(M^{(s)}, \theta_{\underline{\alpha}}\right)=\operatorname{JType}\left(M, \theta_{F^{s} \circ \underline{\alpha}}\right),
$$

where $F^{s} \circ \underline{\alpha}=\left(0, \ldots, 0, \phi^{s}\left(\alpha_{1}\right), \ldots, \phi^{s}\left(\alpha_{r-1-s}\right)\right)$.

If $M$ is a finite dimensional $k \mathbb{G}_{a(r)}$-module and $\underline{a}=\left(a_{0}, \ldots, a_{r-1}\right)$ is a point in $V\left(\mathbb{G}_{a(r)}\right) \simeq \mathbb{A}^{r}$, then

$$
\operatorname{JType}\left(M^{(s)}, \theta_{\underline{a}}\right)=\operatorname{JType}\left(M, \theta_{F^{s} \circ \underline{a}}\right),
$$

where $F^{s} \circ \underline{a}=\left(0, \ldots, 0, a_{0}^{p^{s}}, \ldots, a_{r-1-s}^{p^{s}}\right)$.

Proposition 3.10 has the following immediate corollary (see Remark 3.3).

Corollary 3.11. Let $M$ be a finite dimensional representation of $\mathrm{GL}_{n(r)}$. Then $\underline{\alpha}=\left(\alpha_{0}, \ldots, \alpha_{r-1}\right) \in V\left(\mathrm{GL}_{n(r)}\right)_{M}$ if and only if

$$
F^{s} \circ \alpha=\left(0, \ldots, 0, \phi^{s}\left(\alpha_{0}\right), \ldots, \phi^{s}\left(\alpha_{r-1-s}\right)\right) \in V\left(\mathrm{GL}_{n(r)}\right)_{M^{(s)}} .
$$

The following definition introduces interesting classes of $k G$-modules which have special local behavior. 
Definition 3.12. Let $G$ be an infinitesimal group scheme and $j$ a positive integer less than $p$. A finite dimensional $k G$-module $M$ is said to be of constant $j$-rank if and only if

$$
\operatorname{rk}\left(M, \theta_{v}^{j}\right) \equiv \operatorname{rk}\left\{\theta_{v}^{j}: M_{k(v)} \rightarrow M_{k(v)}\right\}
$$

is independent of $v \in V(G)-\{0\}$, where $\theta_{v}$ is the local $p$-nilpotent operator at $v$ as introduced in Definition 3.1.

$M$ is said to be of constant Jordan type if and only if it is of constant $j$-rank for all $j, 1 \leq j<p . M$ is said to be of constant rank if it is of constant 1-rank.

As we see in the following example, one can have rational $\mathrm{GL}_{n}$-modules of constant Jordan type when restricted to $\mathrm{GL}_{n(r)}$ of arbitrarily high degree $d$. This should be contrasted with Corollary 3.17.

Example 3.13. Consider the rational $\mathrm{GL}_{n}$-module $M=\operatorname{det}^{\otimes d}$, the $d^{\text {th }}$ power of the determinant representation for some $d>0$. This is a polynomial representation of degree $n^{d}$. The restriction of $M$ to any Frobenius kernel $\mathrm{GL}_{n(r)}$ has (trivial) constant Jordan type, for the further restriction of $M$ to any abelian unipotent subgroup of $\mathrm{GL}_{n}$ has trivial action.

We shall see below that $k G$-modules of constant $j$-rank lead to interesting constructions of vector bundles (see Theorem 5.1). We conclude this section by establishing two constraints, Propositions 3.15 and 3.19 , on $k G$-modules to be modules of constant rank.

We first need the following elementary lemma.

Lemma 3.14. Let $M$ be a $\mathbb{G}_{a(r)}$-module such that the local Jordan type at every $v \in V\left(\mathbb{G}_{a(r)}\right)$ is trivial. Then $M$ is trivial as a $k \mathbb{G}_{a(r)}$-module.

Proof. The action of $\mathbb{G}_{a(r)}$ on $M$ is given by the action of $r$ commuting $p$-nilpotent operators $\tilde{u}_{i}, 0 \leq i<p$, on $M$. Moreover, for $\underline{a}=(0, \ldots, 1,0, \ldots, 0)$, with 1 at the $i^{\text {th }}$ spot,

$$
\operatorname{JType}\left(M, \theta_{\underline{a}}\right)=\operatorname{JType}\left(M, \tilde{u}_{i}\right),
$$

as follows from the explicit description of $\Theta_{\mathbb{G}_{a(r)}}$ in Example 2.6(2). Thus, if the local Jordan type of $M$ is trivial at each $\underline{a}=(0, \ldots, 1,0, \ldots, 0)$, then each $\tilde{u}_{i}$ must act trivially on $M$, and $M$ is therefore a trivial $\mathbb{G}_{a(r)}$-module.

Proposition 3.15. Let $\mathfrak{G}$ be an algebraic group generated by 1-parameter subgroups $i: \mathbb{G}_{a} \subset \mathfrak{G}$. Let $\rho: \mathfrak{G} \rightarrow \mathrm{GL}_{N}$ determine a non-trivial rational representation $M$ of $\mathfrak{G}$. Let $D_{i}$ be the minimum of the degrees of $(\rho \circ i)^{*}\left(X_{s, t}\right) \in k\left[\mathbb{G}_{a}\right]=k[T]$ as $X_{s, t}$ ranges over the matrix coordinate functions of $\mathrm{GL}_{N}$. Let $D=\max \left\{D_{i}, i: \mathbb{G}_{a} \subset \mathfrak{G}\right\}$.

If $r>\log _{p} D+1$, then $M$ is not of constant Jordan type as a $\mathfrak{G}_{(r)}$-module.

Proof. Because $M$ is non-trivial and $\mathfrak{G}$ is generated by its 1-parameter subgroups, we conclude that $i^{*} M$ is a non-trivial rational $\mathbb{G}_{a}$ representation for some 1-parameter subgroup $i: \mathbb{G}_{a} \subset \mathfrak{G}$. The condition $r>\log _{p} D \geq \log _{p} D_{i}$ implies that $i^{*} M$ is not $r$-twisted (i.e., of the form $N^{(r)}$ ). Lemma 3.14 implies that the local Jordan type of $i^{*} M$ at some 1-parameter subgroup $\mu_{v}: \mathbb{G}_{a(r), k(v)} \rightarrow G_{k(v)}$ is non-trivial. On the other hand, Corollary 3.8 implies that the Jordan type of $i^{*} M$ at the identity 1-parameter subgroup id : $\mathbb{G}_{a(r)} \rightarrow \mathbb{G}_{a(r)}$ is trivial provided that $r-1>\log _{p} D$.

As an immediate corollary, we conclude the following. 
Corollary 3.16. Let $\mathfrak{G}$ be an algebraic group generated by 1-parameter subgroups and let $M$ be a non-trivial rational representation of $\mathfrak{G}$. Then for $r \gg 0, M$ is not of constant Jordan type as a $k \mathfrak{G}_{(r)}$-module.

As an explicit example of Proposition [3.15, we obtain the following corollary (which should be contrasted with Example 3.13).

Corollary 3.17. Let $M$ be a non-trivial polynomial representation of $\mathrm{SL}_{n}$ of degree $D$. If $r>\log _{p} D+1$, then $M$ is not a $k \mathrm{SL}_{n(r)}$-module of constant rank.

The following lemma, which is a straightforward application of the Generalized Principal Ideal Theorem (see [12, 10.9]), shows that the dimension of a non-trivial module of constant rank of $\mathbb{G}_{a(r)}$ cannot be "too small" compared to $r$.

Lemma 3.18. Let $M$ be a finite dimensional $\mathbb{G}_{a(r)}$-module. If $M$ is a non-trivial $\mathbb{G}_{a(r)}$-module of constant rank, then the following inequality holds:

$$
\operatorname{dim}_{k} M \geq \sqrt{r} .
$$

Proof. By extending scalars if necessary we may assume that $k$ is algebraically closed. Let $m=\operatorname{dim}_{k} M$. Let $k \mathbb{G}_{a(r)}=k\left[u_{0}, \ldots, u_{r-1}\right] /\left(u_{0}^{p}, \ldots, u_{r-1}^{p}\right)$, let $K=$ $k\left(s_{0}, \ldots, s_{r-1}\right)$ where $s_{i}$ are independent variables, and let $\alpha_{K}: K[t] / t^{p} \rightarrow K \mathbb{G}_{a(r)}$ be a map of $K$-algebras defined by $\alpha_{K}(t)=s_{0} u_{0}+\cdots+s_{r-1} u_{r-1}$. Choose a $k$-linear basis of $M$, and let $A\left(s_{0}, \ldots, s_{r-1}\right)$ be a nilpotent matrix in $M_{m}\left(k\left[s_{0}, \ldots, s_{r-1}\right]\right)$ representing the action of $\alpha_{K}(t)$ on $M_{K}$. Let $I_{n}\left(A\left(s_{0}, \ldots, s_{r-1}\right)\right)$ denote the ideal generated by all $n \times n$ minors of $A\left(s_{0}, \ldots, s_{r-1}\right)$. By [12, 10.9], the codimension of any minimal prime over $I_{n}\left(A\left(s_{0}, \ldots, s_{r-1}\right)\right)$ is at most $(m-n+1)^{2}$.

Assume that (3.18.1) does not hold, that is, $m<\sqrt{r}$. Hence, $(m-n+1)^{2}<r$ for any $1 \leq n \leq m$. The variety of $I_{n}\left(A\left(s_{0}, \ldots, s_{r-1}\right)\right)$ is a subvariety inside Spec $k\left[s_{0}, \ldots, s_{r-1}\right] \simeq \mathbb{A}^{r}$ which has dimension $r$. Since the codimension of the variety of $I_{n}\left(A\left(s_{0}, \ldots, s_{r-1}\right)\right)$ is at most $(m-n+1)^{2}$, we conlude that the dimension is at least $r-(m-n+1)^{2} \geq 1$. Hence, the minors of dimension $n \times n$ have a common non-trivial zero. Taking $n=1$, we conclude that $A\left(b_{0}, \ldots, b_{r-1}\right)$ is a zero matrix for some non-zero specialization $b_{0}, \ldots, b_{r-1}$ of $s_{0}, \ldots, s_{r-1}$. Consequently, $M$ is trivial at the $\pi$-point of $\mathbb{G}_{a(r)}$ corresponding to $b_{0}, \ldots, b_{r-1}$. Since $M$ is non-trivial, Lemma 3.14 implies that $M$ is not a module of constant rank.

As an immediate corollary, we provide an additional necessary condition for a $k \mathfrak{G}_{(r)}$-module to have constant rank.

Proposition 3.19. Let $\mathfrak{G}$ be a (reduced) affine algebraic group and $M$ be a rational representation of $\mathfrak{G}$. Assume that $\mathfrak{G}$ admits a 1-parameter subgroup $\mu: \mathbb{G}_{a(r)} \rightarrow \mathfrak{G}$ such that $\mu^{*}(M)$ is a non-trivial $k \mathbb{G}_{a(r)}$-module. If $r \geq(\operatorname{dim} M)^{2}+1$, then $M$ is not a $k \mathfrak{G}_{(r)}$-module of constant rank.

$$
\text { 4. } \pi \text {-POINTS AND } \mathbb{P}(\mathrm{G})
$$

In a series of earlier papers, we have considered $\pi$-points for a finite group scheme $G$ (as recalled in Definition 4.1) and investigated finite dimensional $k G$-modules $M$ using the "Jordan type of $M$ " at various $\pi$-points. In particular, in [18, we verified that this Jordan type is independent of the equivalence class of the $\pi$-point provided that either the $\pi$-point is generic or the Jordan type of $M$ at some representative of the equivalence class is maximal. 
As we recall below, whenever $G$ is an infinitesimal group scheme, the $\pi$-point space $\Pi(G)$ of equivalence classes of $\pi$-points is essentially the projectivization of $V(G)$. The purpose of the first half of this section is to relate the discussion of the previous section concerning the local Jordan type of a finite $k G$-module to our earlier work formulated in terms of $\pi$-points for general finite group schemes.

One special aspect of an infinitesimal group scheme $G$ is that equivalence classes of $\pi$-points of $G$ have canonical (up to scalar multiple) representatives.

Unless otherwise specified (as in Definition 4.1 immediately below), $G$ will denote an infinitesimal group scheme over $k$, and $V(G)$ will denote $V_{r}(G)$ for some $r \geq$ ht(G). Throughout this section we assume that $\operatorname{dim} V(G) \geq 1$, and we work with $\mathbb{P}(\mathrm{G})=\operatorname{Proj} k[V(G)]$. We note that if $\operatorname{dim} V(G)=0$, then Theorem 1.16 implies that the projective resolution of the trivial module $k$ is finite. Since $k G$ is selfinjective, this further implies that $k$ is projective. Hence, $k G$ is semi-simple and does not have any $\pi$-points (see, for example, [15, §2]).

Definition 4.1 (see [17]). Let $G$ be a finite group scheme.

(1) A $\pi$-point of $G$ is a (left) flat map of $K$-algebras $\alpha_{K}: K[t] / t^{p} \rightarrow K G$ for some field extension $K / k$ with the property that there exists a unipotent abelian closed subgroup scheme $i: C_{K} \subset G_{K}$ defined over $K$ such that $\alpha_{K}$ factors through $i_{*}: K C_{K} \rightarrow K G_{K}=K G$.

(2) If $\beta_{L}: L[t] / t^{p} \rightarrow L G$ is another $\pi$-point of $G$, then $\alpha_{K}$ is said to be a specialization of $\beta_{L}$, written $\beta_{L} \downarrow \alpha_{K}$, provided that for any finite dimensional $k G$-module $M, \alpha_{K}^{*}\left(M_{K}\right)$ being free as a $K[t] / t^{p}$-module implies that $\beta_{L}^{*}\left(M_{L}\right)$ is free as an $L[t] / t^{p}$-module.

(3) Two $\pi$-points $\alpha_{K}: K[t] / t^{p} \rightarrow K G, \beta_{L}: L[t] / t^{p} \rightarrow L G$ are said to be equivalent, written $\alpha_{K} \sim \beta_{L}$, if $\alpha_{K} \downarrow \beta_{L}$ and $\beta_{L} \downarrow \alpha_{K}$.

(4) A $\pi$-point of $G, \alpha_{K}: K[t] / t^{p} \rightarrow K G$, is said to be generic if there does not exist another $\pi$-point $\beta_{L}: L[t] / t^{p} \rightarrow L G$ which specializes to $\alpha_{K}$ but is not equivalent to $\alpha_{K}$.

(5) If $M$ is a finite dimensional $k G$-module and $\alpha_{K}: K[t] / t^{p} \rightarrow K G$ a $\pi$-point of $G$, then the Jordan type of $M$ at $\alpha_{K}$ is by definition the Jordan type of $\alpha_{K}^{*}\left(M_{K}\right)$ as a $K[t] / t^{p}$-module.

Because the group algebra of a finite group scheme is always faithfully flat over the group algebra of a subgroup scheme (see [27, 14.1]), the condition on a flat map $\alpha_{K}: K[t] / t^{p} \rightarrow K G$ is equivalent to the existence of a factorization $i_{*} \circ \alpha_{K}^{\prime}$ with $\alpha_{K}^{\prime}: K[t] / t^{p} \rightarrow K C_{K}$ flat.

Definition 4.2. Let $G$ be an infinitesimal group scheme, and let $v \in V(G)$ be the point associated to the 1-parameter subgroup $\mu_{v}: \mathbb{G}_{a(r), k(v)} \rightarrow G_{k(v)}$. Then the $\pi$-point of $G$ associated to $v$ is

$$
\mu_{v, *} \circ \epsilon: k(v)[u] / u^{p} \rightarrow k(v) G,
$$

where $\epsilon: k(v)[u] / u^{p} \rightarrow k(v) \mathbb{G}_{a(r), k(v)}$ is as defined in (1.14.1).

The following theorem is a complement to Theorem 1.16, revealing that spaces of (equivalence) classes of $\pi$-points are very closely related to (cohomological) support varieties.

Theorem 4.3 ([17, 7.5]). Let $G$ be a finite group scheme. Then the set of equivalence classes of $\pi$-points, denoted $\Pi(G)$, can be given a scheme structure, which is 
defined in terms of the representation theory of $G$. Moreover, there is an isomorphism of schemes

$$
\text { Proj } \mathrm{H}^{\bullet}(G, k) \simeq \Pi(G) .
$$

If $G$ is an infinitesimal group scheme so that $\mathrm{H}^{\bullet}(G, k)$ is related to $k[V(G)]$ as in Theorem 1.16, then the resulting homeomorphism

$$
\mathbb{P}(G) \equiv \operatorname{Proj} k[V(G)] \longrightarrow \operatorname{Proj}^{\bullet}(G, k) \simeq \Pi(G)
$$

is given on points by sending $x \in \mathbb{P}(G)$ to the equivalence class of the $\pi$-point $\mu_{v, *} \circ \epsilon$ for any $v \in V(G) \backslash\{0\}$ projecting to $x$. In particular, equivalence classes of generic $\pi$-points of $G$ are represented by $\left(\mu_{v, *} \circ \epsilon\right)$ as $v \in V(G)$ runs through the (scheme-theoretic) generic points of $V(G)$.

Furthermore, for any finite dimensional $k G$-module $M$, (4.3.1) restricts to a homeomorphism of subvarieties

$$
\mathbb{P}(G)_{M} \simeq \Pi(G)_{M},
$$

where $\mathbb{P}(G)_{M}=\operatorname{Proj}\left(k\left[V(G)_{M}\right]\right)$ and $\Pi(G)_{M}$ consists of those equivalence classes of $\pi$-points $\alpha_{K}$ of $G$ such that $\alpha_{K}^{*}\left(M_{K}\right)$ is not free (as a $K[u] / u^{p}$-module).

Generic $\pi$-points are particularly important when developing invariants of representations. The following corollary of Theorem 4.3 gives an explicit set of representatives of equivalence classes of generic $\pi$-points of $G$.

Proposition 4.4. Let $G$ be an infinitesimal group scheme with universal 1-parameter subgroup $\mathcal{U}_{G}: \mathbb{G}_{a(r), k[V(G)]} \longrightarrow G_{k[V(G)]}$. For each minimal prime ideal $\mathcal{P}_{i}$ of $k[V(G)]$, let $K_{i}$ denote the field of fractions of $k[V(G)] / \mathcal{P}_{i}$. Then the compositions

$$
\left(\mathcal{U}_{G, *} \otimes_{k[V(G)]} K_{i}\right) \circ \epsilon: K_{i}[u] / u^{p} \rightarrow K_{i} G
$$

(sending $u$ to $\theta_{K_{i}}$ ) are non-equivalent representatives of the equivalence classes of generic $\pi$-points of $G$.

To obtain vector bundles, we require the following well known, elementary observation about commutative graded algebras.

Lemma 4.5. Let $A$ be a finitely generated commutative, graded $k$-algebra with homogeneous generators whose degrees divide $d$ and let $X=\operatorname{Proj} A$. Then $\mathcal{O}_{X}(d)$ is a locally free sheaf of rank 1 on $X$.

Proof. Let $\left\{f_{i}\right\}$ be a finite set of homogeneous generators of $A$, set $d_{i}$ equal to the degree of $f_{i}$, and choose $d$ such that each $d_{i}$ divides $d$. Set $U_{i}=X-Z\left(f_{i}\right)$, an affine open subset of $X$ with coordinate algebra $\left(A_{f_{i}}\right)_{0}$, the degree zero subalgebra of the localization of $A$ obtained by inverting $f_{i}$. Then multiplication by $f_{i}^{d / d_{i}}$ induces an isomorphism $\left(\mathcal{O}_{X}\right)_{\mid U_{i}} \stackrel{\sim}{\rightarrow}\left(\mathcal{O}_{X}(d)\right)_{\mid U_{i}}$. Thus the restriction of $\mathcal{O}_{X}(d)$ to each $U_{i}$ of the open covering $\left\{U_{i}\right\}$ is free of rank 1 .

Let $G$ be an infinitesimal group scheme of height $\leq r$ and recall from Proposition 2.11 that $\Theta_{G, r} \in k G \otimes k\left[V_{r}(G)\right]$ is homogeneous of degree $p^{r-1}$.

Definition 4.6. Let $G$ be an infinitesimal group scheme of height $\leq r$ and let $M$ be a finite dimensional $k G$-module. Then we denote by

$$
\widetilde{\Theta}_{G}: \mathcal{M} \equiv M \otimes \mathcal{O}_{\mathbb{P}(\mathrm{G})} \longrightarrow \mathcal{M}\left(p^{r-1}\right) \equiv M \otimes \mathcal{O}_{\mathbb{P}(\mathrm{G})}\left(p^{r-1}\right)
$$


the homomorphism of (locally free) coherent $\mathcal{O}_{\mathbb{P}(\mathrm{G})}$-modules determined by the action of $\Theta_{G, r} \in k G \otimes k\left[V_{r}(G)\right]$.

We denote by

$$
\widetilde{\Theta}_{G}(n): \mathcal{M}(n) \longrightarrow \mathcal{M}\left(p^{r-1}+n\right)
$$

the map obtained by tensoring (4.6.1) with $\mathcal{O}_{\mathbb{P}(G)}(n)$.

For any point $x \in \mathbb{P}(\mathrm{G})$, we use the notation

$$
M_{k(x)}=\mathcal{M} \otimes_{\mathcal{O}_{\mathbb{P}(G)}} k(x)
$$

for the fiber of the coherent sheaf $\mathcal{M}$ at $x$. Here, we have identified $k(x)$ with the residue field of the stalk $\mathcal{O}_{\mathbb{P}(\mathrm{G}), x}$.

Proposition 4.7. Let $G$ be an infinitesimal group scheme of height $\leq r$, and let $M$ be a finite dimensional $k G$-module. For any $v, v^{\prime} \in V(G)$ projecting to the same $x \in \mathbb{P}(\mathrm{G})$, we have

$$
\operatorname{Im}\left\{\theta_{v}: M_{k(v)} \rightarrow M_{k(v)}\right\} \simeq \operatorname{Im}\left\{\theta_{v^{\prime}}: M_{k\left(v^{\prime}\right)} \rightarrow M_{k\left(v^{\prime}\right)}\right\}
$$

and similarly for kernels.

Proof. This is essentially proved in [26, 6.1].

In the next section, we shall be particularly interested in kernels and images of $\widetilde{\Theta}_{G}$. The following proposition relates the fibers of the kernel and image of the global $p$-nilpotent operator $\widetilde{\Theta}_{G}$ at a point $x \in \mathbb{P}(G)$ with the kernel and image of the local $p$-nilpotent operator $\theta_{v}$ on $M \otimes k(v)$ for $v$ representing $x$.

Proposition 4.8. Let $G$ be an infinitesimal group scheme of height $\leq r$, let $M$ be a finite dimensional $k G$-module, let $\mathcal{M}=M \otimes \mathcal{O}_{\mathbb{P}(\mathrm{G})}$, and let $s \in \Gamma\left(\mathbb{P}(\mathrm{G}), \mathcal{O}_{\mathbb{P}(\mathrm{G})}\left(p^{r-1}\right)\right)$ be a non-zero global section with zero locus $Z(s) \subset \mathbb{P}(\mathrm{G})$. Set $U=\mathbb{P}(\mathrm{G}) \backslash Z(s)$. Then there is a well defined endomorphism (depending upon $s$ )

$$
\widetilde{\Theta}_{G} / s: \mathcal{M}_{\mid U} \rightarrow \mathcal{M}_{\mid U} .
$$

Moreover, the image and kernel of the induced map $\theta_{x} / s: M_{k(x)} \rightarrow M_{k(x)}$ on fibers at $x \in U \subset \mathbb{P}(\mathrm{G})$ is independent of $s$ and satisfies

$$
\operatorname{Im}\left\{\theta_{x} / s: M_{k(x)} \rightarrow M_{k(x)}\right\} \simeq \operatorname{Im}\left\{\theta_{v}: M_{k(v)} \rightarrow M_{k(v)}\right\}
$$

and

$$
\operatorname{Ker}\left\{\theta_{x} / s: M_{k(x)} \rightarrow M_{k(x)}\right\} \simeq \operatorname{Ker}\left\{\theta_{v}: M_{k(v)} \rightarrow M_{k(v)}\right\}
$$

for any $v \in V(G) \backslash\{0\}$ that projects onto $x$.

Proof. Let $X=\mathbb{P}(\mathrm{G})$ and let $\frac{1}{s} \in \mathcal{O}_{X}\left(-p^{r-1}\right)(U)$ satisfy

$$
s \otimes \frac{1}{s}=1 \in \mathcal{O}_{X}\left(p^{r-1}\right)(U) \otimes_{\mathcal{O}_{X}(U)} \mathcal{O}_{X}\left(-p^{r-1}\right)(U) \simeq \mathcal{O}_{X}(U) .
$$

Then we define

$$
\widetilde{\Theta}_{G} / s \equiv\left(\widetilde{\Theta}_{G}\right)_{\mid U} \otimes \frac{1}{s}: \mathcal{M}_{\mid U} \rightarrow \mathcal{M}\left(p^{r-1}\right)_{\mid U} \otimes \mathcal{O}_{X}\left(-p^{r-1}\right)_{\mid U} \simeq \mathcal{M}_{\mid U}
$$

Let $A=k[V(G)]$. Then $U$ is an affine subscheme of $\mathbb{P}(\mathrm{G})$ determined by the 0-degree elements of $A\left[\frac{1}{s}\right], U \simeq \operatorname{Spec} A\left[\frac{1}{s}\right]_{0}$. Via this identification, $\mathcal{M}_{\mid U}$ corresponds to the free $A\left[\frac{1}{s}\right]_{0}$-module $M \otimes A\left[\frac{1}{s}\right]_{0}$. The fiber $M_{k(x)}$ of $\mathcal{M}_{\mid U}$ at the point $x$ is naturally identified with the fiber of $M \otimes A\left[\frac{1}{s}\right]_{0}$ at $x$. Since 
$\Theta_{G}: M \otimes k[V(G)] \rightarrow M \otimes k[V(G)]$ is homogeneous of degree $p^{r-1}$ and $s \in k[V(G)]$ is homogeneous of the same degree, the operator $\Theta_{G} \otimes \frac{1}{s}$ is well defined on $M \otimes A\left[\frac{1}{s}\right]_{0}$ and corresponds to the operator $\widetilde{\Theta}_{G} / s$ on $\mathcal{M}_{\mid U}$. Hence, $\theta_{x} / s: M_{k(x)} \rightarrow M_{k(x)}$ is identified with

$$
\left(\widetilde{\Theta}_{G} \otimes \frac{1}{s}\right) \otimes k(x)=\frac{\Theta_{G}}{s}(x): M_{k(x)} \rightarrow M_{k(x)} .
$$

Let $v \in V(G)$ be any point projecting onto $x$. We have $k(x)=k(v)$. By Definition 3.1, the map $\theta_{v}: M_{k(v)} \rightarrow M_{k(v)}$ is given by

$$
\Theta_{G}(v): M_{k(v)} \rightarrow M_{k(v)} .
$$

We observe that $\frac{\Theta_{G}}{s}(x)=\frac{\Theta_{G}(v)}{s(v)}$ for any $v$ projecting onto $x$ (and, in particular, is independent of the choice of $v$ ). Therefore,

$$
\theta_{x} / s=\frac{\theta_{v}}{s(v)} .
$$

The equalities 4.8.2) and (4.8.1) now follow.

Remark 4.9. For a finite group $G$, there is no natural choice of $\pi$-point representing a typical equivalence class $x \in \Pi(G) \simeq \operatorname{Proj} \mathrm{H}^{\bullet}(G, k)$ of $\pi$-points. As seen in elementary examples [18, 2.3], the Jordan type of a $k G$-module $M$ typically can be different for two equivalent $\pi$-points representing the same point $x \in \Pi(G)$.

Remark 4.10. Proposition 4.8 immediately generalizes to $\widetilde{\Theta}_{G}^{j}$ for any $1 \leq j \leq p-1$. Thus, we have the following isomorphisms for any $x \in X=\mathbb{P}(\mathrm{G}), v \in V(G)$ projecting onto $x$, and a global section $s$ of $\mathcal{O}_{X}\left(j p^{r-1}\right)$ such that $s(x) \neq 0$ :

$$
\begin{aligned}
& \operatorname{Im}\left\{\left(\theta_{x} / s\right)^{j}: M_{k(x)} \rightarrow M_{k(x)}\right\} \simeq \operatorname{Im}\left\{\theta_{v}^{j}: M_{k(v)} \rightarrow M_{k(v)}\right\} \\
\simeq & \operatorname{Im}\left\{\widetilde{\Theta}_{G}^{j} \otimes_{\mathcal{O}_{X}} k(x): \mathcal{M} \otimes_{\mathcal{O}_{X}} k(x) \rightarrow \mathcal{M}\left(j p^{r-1}\right) \otimes_{\mathcal{O}_{X}} k(x)\right\},
\end{aligned}
$$

and similarly for kernels.

In what follows, we shall use the following abbreviations:

$$
\begin{aligned}
& \operatorname{Im}\left\{\widetilde{\Theta}_{G}^{j}, \mathcal{M}\right\} \equiv \operatorname{Im}\left\{\widetilde{\Theta}_{G}^{j}\left(-j p^{r-1}\right): \mathcal{M}\left(-j p^{r-1}\right) \rightarrow \mathcal{M}\right\}, \\
& \operatorname{Im}\left\{\theta_{x}^{j}, M_{k(x)}\right\} \equiv \operatorname{Im}\left\{\left(\theta_{x} / s\right)^{j}: M_{k(x)} \rightarrow M_{k(x)}\right\}, \\
& \operatorname{Ker}\left\{\widetilde{\Theta}_{G}^{j}, \mathcal{M}\right\} \equiv \operatorname{Ker}\left\{\widetilde{\Theta}_{G}^{j}: \mathcal{M} \rightarrow \mathcal{M}\left(j p^{r-1}\right)\right\}, \\
& \operatorname{Ker}\left\{\theta_{x}^{j}, M_{k(x)}\right\} \equiv \operatorname{Ker}\left\{\left(\theta_{x} / s\right)^{j}: M_{k(x)} \rightarrow M_{k(x)}\right\}, \\
& \operatorname{Coker}\left\{\widetilde{\Theta}_{G}^{j}, \mathcal{M}\right\} \equiv \mathcal{M} / \operatorname{Im}\left\{\widetilde{\Theta}_{G}^{j}, \mathcal{M}\right\}, \\
& \operatorname{Coker}\left\{\theta_{x}^{j}, M_{k(x)}\right\} \equiv \operatorname{Coker}\left\{\left(\theta_{x} / s\right)^{j}: M_{k(x)} \rightarrow M_{k(x)}\right\} .
\end{aligned}
$$

Note that both Ker and Im are subsheaves of the free sheaf $\mathcal{M}$ and that Coker is a quotient sheaf of $\mathcal{M}$.

We shall verify in Theorem 4.13 that a necessary and sufficient condition on a finite dimensional $k G$-module $M$ for $\operatorname{Im}\left\{\widetilde{\Theta}_{G}^{j}, \mathcal{M}\right\}$ (and thus $\operatorname{Ker}\left\{\widetilde{\Theta}_{G}^{j}, \mathcal{M}\right\}$ ) to be an algebraic vector bundle on $X$ is that $M$ be a module of constant $j$-type.

The following proposition is given in [20, 5, ex. 5.8] without proof.

Proposition 4.11. Let $X$ be a reduced scheme and $\widetilde{M}$ a coherent $\mathcal{O}_{X}$-module. Then $\widetilde{M}$ is locally free if and only if $\operatorname{dim}_{k(x)}\left(\widetilde{M} \otimes_{\mathcal{O}_{X}} k(x)\right)$ depends only upon the connected component of $x$ in $\pi_{0}(X)$. 
Proof. Assume that the function $x \mapsto \operatorname{dim}_{k(x)}\left(\widetilde{M} \otimes_{\mathcal{O}_{X}} k(x)\right)$ is constant on each connected component of $X$. To prove that $\widetilde{M}$ is locally free it suffices to assume that $X$ is local so that $X=\operatorname{Spec} R$ for some reduced local commutative ring, and that $M$ is a finite $R$-module (corresponding to the coherent sheaf $\widetilde{M}$ ) with the property that $\operatorname{dim}_{k(p)}\left(M \otimes_{R} k(p)\right)$ is independent of the prime $p \subset R$. To prove that $M$ is free, we choose some surjective $R$-module homomorphism $g: Q \rightarrow M$ from a free $R$-module $Q \simeq R^{n}$ with the property that $\bar{g}: Q \otimes_{R} R / \mathfrak{m} \rightarrow M \otimes_{R} R / \mathfrak{m}$ is an isomorphism where $\mathfrak{m} \subset R$ is the maximal ideal. Then $g$ is surjective by Nakayama's Lemma. By assumption, $g$ induces an isomorphism after specialization to any prime $\mathfrak{p} \subset R$ : $Q \otimes_{R_{\mathfrak{p}}} k(\mathfrak{p}) \simeq M \otimes_{R_{\mathfrak{p}}} k(\mathfrak{p})$. Hence, $Q_{\mathfrak{p}} / \mathfrak{p} Q_{\mathfrak{p}} \simeq M_{\mathfrak{p}} / \mathfrak{p} M_{\mathfrak{p}}$. We conclude that if $a \in \operatorname{ker} g$, then $a \in \mathfrak{p} Q_{\mathfrak{p}} \cap Q$. Since this happens for any prime ideal, we further conclude that ker $g \subset\left(\bigcap_{\mathfrak{p} \in \operatorname{Spec} R} \mathfrak{p} Q_{\mathfrak{p}}\right) \cap Q$. Recall that $Q$ is a free module so that $Q \simeq R^{n}$. We get $\left(\bigcap \mathfrak{p} Q_{\mathfrak{p}}\right) \cap Q=\left(\bigcap \mathfrak{p} R_{\mathfrak{p}}^{n}\right) \cap R^{n}=\left(\left(\cap \mathfrak{p} R_{\mathfrak{p}}\right) \cap R\right)^{n}=(\bigcap \mathfrak{p} R)^{n}=0$, since $R$ is reduced.

We shall find it convenient to "localize" the notion of a $k G$-module of constant $j$-rank given in Definition 3.12 as follows.

Definition 4.12. Let $G$ be an infinitesimal group scheme, and let $M$ be a finite dimensional $k G$-module. For any open subset $U \subset \mathbb{P}(\mathrm{G}), M$ is said to be of constant $j$-rank when restricted to $U$ if $\operatorname{rk}_{k(x)}\left(\left(\theta_{x} / s\right)^{j}: M_{k(x)} \rightarrow M_{k(x)}\right)$ is independent of $x \in U$.

Our next theorem emphasizes the local nature of the concept of constant $j$-rank.

Theorem 4.13. Let $G$ be an infinitesimal group scheme, let $M$ be a finite dimensional $k G$-module, and let $X=\mathbb{P}(G)$. Let $U \subset X$ be a connected open subset and $\widetilde{\Theta}_{U}^{j}: \mathcal{M}_{\mid U} \rightarrow \mathcal{M}\left(j p^{r-1}\right)_{\mid U}$ be the restriction to $U$ of the $j^{\text {th }}$ iterate of $\widetilde{\Theta}_{G}$ on $\mathcal{M}=M \otimes \mathcal{O}_{X}$ as given in (4.6.1). Then the following are equivalent for some fixed $j, 1 \leq j<p$ :

(1) $\operatorname{Im}\left\{\widetilde{\Theta}_{U}^{j}, \mathcal{M}_{\mid U}\right\}$ is a locally free, coherent $\mathcal{O}_{U}$-module.

(2) $\operatorname{Im}\left\{\widetilde{\Theta}_{G}^{j}, \mathcal{M}\right\} \otimes_{\mathcal{O}_{X}} k(x)$ has dimension independent of $x \in U$.

(3) $\operatorname{Im}\left\{\theta_{x}^{j}, M_{k(x)}\right\} \simeq \operatorname{Im}\left\{\widetilde{\Theta}_{G}^{j}, \mathcal{M}\right\} \otimes_{\mathcal{O}_{X}} k(x), \forall x \in U$.

(4) $M$ has constant $j$-rank when restricted to $U$.

Moreover, each of these conditions implies that

(5) $\operatorname{Coker}\left\{\widetilde{\Theta}_{U}^{j}, \mathcal{M}_{U}\right\}$ is a locally free, coherent $\mathcal{O}_{U}$-module.

(6) $\operatorname{Coker}\left\{\theta_{x}^{j}, M_{k(x)}\right\} \simeq \operatorname{Coker}\left\{\widetilde{\Theta}_{G}^{j}, \mathcal{M}\right\} \otimes_{\mathcal{O}_{X}} k(x), \forall x \in U$.

(7) $\operatorname{Ker}\left\{\widetilde{\Theta}_{U}^{j}, \mathcal{M}_{U}\right\}$ is a locally free, coherent $\mathcal{O}_{U}$-module.

(8) $\operatorname{Ker}\left\{\theta_{x}^{j}, M_{k(x)}\right\} \simeq \operatorname{Ker}\left\{\widetilde{\Theta}_{G}^{j}, \mathcal{M}\right\} \otimes_{\mathcal{O}_{X}} k(x), \forall x \in U$.

Proof. Clearly, (1) implies (2), whereas Proposition 4.11 implies that (2) implies (1).

If we assume (1), we obtain a locally split short exact sequence of coherent $\mathcal{O}_{U}$-modules

$$
0 \rightarrow \operatorname{Ker}\left\{\widetilde{\Theta}_{U}^{j}, \mathcal{M}_{\mid U}\right\} \rightarrow \mathcal{M}_{\mid U} \rightarrow \operatorname{Im}\left\{\widetilde{\Theta}_{U}^{j}, \mathcal{M}_{\mid U}\right\} \rightarrow 0 .
$$


In particular, $\operatorname{Ker}\left\{\widetilde{\Theta}_{U}^{j}, \mathcal{M}_{\mid U}\right\}$ is a locally free, coherent $\mathcal{O}_{U}$-module. Locally on $U$, $\widetilde{\Theta}_{U}^{j}$ on $\mathcal{M}_{\mid U}$ is isomorphic to the projection

$$
p r_{2}: \operatorname{Ker}\left\{\widetilde{\Theta}_{U}^{j}, \mathcal{M}_{\mid U}\right\} \oplus \operatorname{Im}\left\{\widetilde{\Theta}_{U}^{j}, \mathcal{M}_{\mid U}\right\} \rightarrow \operatorname{Im}\left\{\widetilde{\Theta}_{U}^{j}, \mathcal{M}_{\mid U}\right\} .
$$

Since $\theta_{x}^{j}$ is the base change via $\mathcal{O}_{U} \rightarrow k(x)$ of $\widetilde{\Theta}_{U}^{j}, \theta_{x}^{j}$ can be identified with the base change of this projection, and thus we may conclude (3).

Let us now assume (3). A simple argument using Nakayama's Lemma as in the proof of Proposition 4.11 implies that the function $x \mapsto \operatorname{Im}\left\{\theta_{x}^{j}, M_{k(x)}\right\}$ is lower semi-continuous on $U$, whereas the function $x \mapsto \operatorname{Im}\left\{\widetilde{\Theta}_{G}^{j}, \mathcal{M}\right\} \otimes_{\mathcal{O}_{X}} k(x)$ is upper semi-continuous on $U$. Thus, we conclude that each of these functions is constant (since $U$ is connected), thereby concluding (2).

Since $\operatorname{rk}\left\{\left(\theta_{x} / s\right)^{j}\right\}=\operatorname{dim}_{k(x)}\left(\operatorname{Im}\left\{\theta_{x}^{j}, M_{k(x)}\right\}\right)$, (2) and (3) imply (4).

Observe that if $f: V \rightarrow V$ is an endomorphism of a finite dimensional vector space, then $\operatorname{dim}\{$ Coker $f\}=\operatorname{dim}\{\operatorname{Ker} f\}$. The assumption that the $k G$-module $M$ has constant rank (i.e., (4)) implies that

$$
\operatorname{dim}_{k(x)}\left(\operatorname{Coker}\left\{\theta_{x}^{j}, M_{k(x)}\right\}\right)=\operatorname{dim}_{k(x)}\left(\operatorname{Ker}\left\{\theta_{x}^{j}, M_{k(x)}\right\}\right)
$$

is independent of $x \in U$. Hence, Proposition 4.11 implies (5). The right exactness of $(-) \otimes_{\mathcal{O}_{X}} k(x)$ applied to

$$
\mathcal{M}\left(-j p^{r-1}\right) \stackrel{\widetilde{\Theta}_{G}^{j}\left(-j p^{r-1}\right)}{\longrightarrow} \mathcal{M} \longrightarrow \operatorname{Coker}\left\{\widetilde{\Theta}_{G}^{j}, \mathcal{M}\right\} \longrightarrow 0
$$

implies (6).

Under the assumption of (5), we obtain a locally split short exact sequence of coherent $\mathcal{O}_{U}$-modules,

$$
0 \rightarrow \operatorname{Im}\left\{\widetilde{\Theta}_{U}^{j}, \mathcal{M}_{\mid U}\right\} \rightarrow \mathcal{M}_{\mid U} \rightarrow \operatorname{Coker}\left\{\widetilde{\Theta}_{U}^{j}, \mathcal{M}_{\mid U}\right\} \rightarrow 0
$$

so that $\operatorname{Im}\left\{\widetilde{\Theta}_{U}^{j}, \mathcal{M}_{\mid U}\right\}$ is a locally free, coherent $\mathcal{O}_{U}$-module. Now, using the short exact sequence of coherent $\mathcal{O}_{U}$-modules,

$$
0 \rightarrow \operatorname{Ker}\left\{\widetilde{\Theta}_{U}^{j}, \mathcal{M}_{\mid U}\right\} \rightarrow \mathcal{M}_{\mid U} \rightarrow \operatorname{Im}\left\{\widetilde{\Theta}_{U}^{j}, \mathcal{M}_{\mid U}\right\} \rightarrow 0,
$$

we conclude that (4) implies (7) (i.e., that $\operatorname{Ker}\left\{\widetilde{\Theta}_{U}^{j}, \mathcal{M}_{\mid U}\right\}$ is locally free). Since the short exact sequence (4.13.1) is locally split, applying $(-) \otimes_{\mathcal{O}_{X}} k(x)$ to (4.13.1) for any $x \in U$ yields a short exact sequence, thereby implying (8).

\section{Vector BundLes FOR MOdUles of CONSTANT $j$-RANK}

In this section, we initiate the study of algebraic vector bundles associated to $k G$-modules of constant $j$-rank as defined in Definition 3.12. Our constructions have two immediate consequences. The first is that certain $k G$-modules with the same "local Jordan type" have non-isomorphic associated vector bundles so that the isomorphism classes of these vector bundles serve as a new invariant. The second is that our construction yields vector bundles on the highly non-trivial projective schemes $\mathbb{P}(\mathrm{G})$.

The reader will find formulas for the ranks of bundles considered, criteria for non-triviality of bundles, a criterion for producing line bundles, a relationship to duality, and another test for the projectivity of $k G$-modules. We also investigate the dimension of global sections of various bundles.

As in Section 4, we assume that $\operatorname{dim} V(G) \geq 1$ throughout this section. 
The special case in which $U=\mathbb{P}(\mathrm{G})$ of Theorem 4.13 is the following assertion that $k G$-modules of constant $j$-rank determine algebraic vector bundles over $\mathbb{P}(\mathrm{G})$.

Theorem 5.1. Let $G$ be an infinitesimal group scheme, let $M$ be a finite dimensional $k G$-module, and let $\mathcal{M}=M \otimes \mathcal{O}_{\mathbb{P}(\mathrm{G})}$ be a free coherent sheaf on $\mathbb{P}(\mathrm{G})$. Then $M$ has constant $j$-rank if and only if $\operatorname{Im}\left\{\widetilde{\Theta}_{G}^{j}, \mathcal{M}\right\}$ is an algebraic vector bundle on $\mathbb{P}(\mathrm{G})$.

Moreover, if $M$ has constant $j$-rank, then $\operatorname{Ker}\left\{\widetilde{\Theta}_{G}^{j}, \mathcal{M}\right\}$ is an algebraic vector bundle on $\mathbb{P}(\mathrm{G})$ as well.

Remark 5.2. Unless $M$ is trivial as a $k G$-module, $\operatorname{Ker}\left\{\Theta_{G}: M \otimes k[V(G)] \rightarrow\right.$ $M \otimes k[V(G)]\}$ is not projective as a $k[V(G)]$-module, since the local $p$-nilpotent operator $\theta_{0}$ at $0 \in V(G)$ is the 0 -map.

We observe the following elementary functoriality of this construction.

Proposition 5.3. Let $i: H \rightarrow G$ be an embedding of infinitesimal group schemes, let $M$ be a finite dimensional $k G$-module, and let $N$ be the restriction of $M$ to $k H$. Let $\mathcal{M}=M \otimes \mathcal{O}_{\mathbb{P}(G)}$ and $\mathcal{N}=N \otimes \mathcal{O}_{\mathbb{P}(H)}$. Then for any $j, 1 \leq j<p$, there are natural isomorphisms of coherent sheaves on $\mathbb{P}(H)$, where $f: \mathbb{P}(H) \rightarrow \mathbb{P}(G)$ is induced by $i$ :

$$
\begin{aligned}
f^{*} \operatorname{Im}\left\{\widetilde{\Theta}_{G}^{j}, \mathcal{M}\right\} & \simeq \operatorname{Im}\left\{\widetilde{\Theta}_{H}^{j}, \mathcal{N}\right\}, \\
f^{*} \operatorname{Ker}\left\{\widetilde{\Theta}_{G}^{j}, \mathcal{M}\right\} & \simeq \operatorname{Ker}\left\{\widetilde{\Theta}_{H}^{j}, \mathcal{N}\right\} .
\end{aligned}
$$

Proof. The statement follows immediately from the commutativity of the diagram

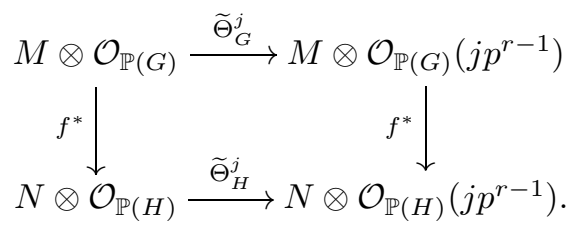

The diagram is commutative by Proposition 2.9

The following corollary will be used later in Section 6 .

Corollary 5.4. Let $G_{1}, G_{2}$ be infinitesimal group schemes, let $G=G_{1} \times G_{2}$, and let $f: \mathbb{P}\left(G_{1}\right) \rightarrow \mathbb{P}(G)$ be the natural embedding of varieties induced by the embedding of group schemes $i: G_{1} \hookrightarrow G$. Let $M_{1}, M_{2}$ be $k G_{1}, k G_{2}$ modules of dimensions $m_{1}, m_{2}$ respectively. Then for any $j, 1 \leq j \leq p$,

$$
f^{*}\left(\operatorname{Ker}\left\{\widetilde{\Theta}_{G}^{j}, \mathcal{M}_{1} \otimes \mathcal{M}_{2}\right\}\right) \simeq \operatorname{Ker}\left\{\widetilde{\Theta}_{G_{1}}^{j}, \mathcal{M}_{1}\right\}^{\oplus m_{2}} .
$$

Here, $\mathcal{M}_{1}=M_{1} \otimes \mathcal{O}_{\mathbb{P}\left(G_{1}\right)}, \mathcal{M}_{2}=M_{2} \otimes \mathcal{O}_{\mathbb{P}\left(G_{2}\right)}$, and $\mathcal{M}_{1} \otimes \mathcal{M}_{2} \simeq\left(M_{1} \otimes M_{2}\right) \otimes \mathcal{O}_{\mathbb{P}(G)}$.

Proof. By Proposition 5.3, it suffices to observe that $\left(M_{1} \otimes M_{2}\right) \downarrow_{G_{1}} \simeq M_{1}^{\oplus m_{2}}$ and that $f^{*}$ and $\widetilde{\Theta}^{j}$ commute with direct sums.

We have a duality for kernel and cokernel bundles. For a vector bundle $\mathcal{E}$ on a projective variety $X$, we denote by $\mathcal{E}^{\vee}=\mathcal{H o m}_{\mathcal{O}_{X}}\left(\mathcal{E}, \mathcal{O}_{X}\right)$ the dual bundle. 
Proposition 5.5. Let $M$ be a finite dimensional $k G$-module of constant $j$-rank. Let $\mathcal{N}=M^{\#} \otimes \mathcal{O}_{\mathbb{P}(\mathrm{G})}$ and $\mathcal{M}=M \otimes \mathcal{O}_{\mathbb{P}(\mathrm{G})}$. Then

$$
\operatorname{Ker}\left\{\widetilde{\Theta}_{G}^{j}, \mathcal{M}\right\}^{\vee} \simeq \operatorname{Coker}\left\{\widetilde{\Theta}_{G}^{j}, \mathcal{N}\right\}
$$

as vector bundles on $\mathcal{O}_{\mathbb{P}(G)}$.

Proof. Choosing dual bases for $M$ and $M^{\#}$, we get an isomorphism of trivial bundles $\mathcal{M}$ and $\mathcal{N}$. Hence, we may identify the dual bundle $\mathcal{M}\left(j p^{r-1}\right)^{\vee}$ with $\mathcal{N}\left(-j p^{r-1}\right)$. Under this identification, the $\mathcal{O}_{\mathbb{P}(G)}$-dual of the map

$$
\widetilde{\Theta}_{G}^{j}: \mathcal{M} \rightarrow \mathcal{M}\left(j p^{r-1}\right)
$$

is identified with

$$
\widetilde{\Theta}_{G}^{j}\left(-j p^{r-1}\right): \mathcal{N}\left(-j p^{r-1}\right) \rightarrow \mathcal{N} .
$$

Since $\operatorname{Ext}_{\mathcal{O}_{\mathbb{P}(\mathrm{G})}^{1}}^{1}\left(-, \mathcal{O}_{\mathbb{P}(\mathrm{G})}\right)$ vanishes on locally free sheaves, taking the $\mathcal{O}_{\mathbb{P}(G)}$-dual of the short exact sequence of vector bundles

$$
0 \longrightarrow \operatorname{Ker}\left\{\widetilde{\Theta}_{G}^{j}, \mathcal{M}\right\} \longrightarrow \mathcal{M} \stackrel{\widetilde{\Theta}_{G}^{j}}{\longrightarrow} \mathcal{M}\left(j p^{r-1}\right) \longrightarrow \operatorname{Coker}\left\{\widetilde{\Theta}^{j}, \mathcal{M}\right\}\left(j p^{r-1}\right) \longrightarrow 0,
$$

we get the exact sequence

$$
\begin{aligned}
0 \longleftarrow \operatorname{Ker}\left\{\widetilde{\Theta}_{G}^{j}, \mathcal{M}\right\}^{\vee} \longleftarrow \mathcal{N} \longleftarrow \Theta_{G}^{j}\left(-j p^{r-1}\right) & \mathcal{N}\left(-j p^{r-1}\right) \\
\longleftarrow & \operatorname{Coker}\left\{\widetilde{\Theta}^{j}, \mathcal{M}\right\}^{\vee}\left(-j p^{r-1}\right) \longleftarrow 0 .
\end{aligned}
$$

Therefore, $\operatorname{Ker}\left\{\widetilde{\Theta}_{G}^{j}, \mathcal{M}\right\}^{\vee} \simeq \operatorname{Coker}\left\{\widetilde{\Theta}_{G}^{j}, \mathcal{N}\right\}$.

Example 5.6. For each of our four examples of infinitesimal group schemes (initially investigated in Example 1.5), we give examples of $k G$-modules of constant Jordan type taken from [8].

(1) Let $\mathfrak{g}$ be a finite dimensional $p$-restricted Lie algebra of dimension at least 2. For any Tate cohomology class of negative dimension, $\zeta \in \widehat{H}^{n}(\mathfrak{u}(\mathfrak{g}), k) \simeq$ $\operatorname{Ext}_{\mathfrak{u}(\mathfrak{g})}^{1}\left(\Omega^{n-1}(k), k\right)$, we consider the extension of $\mathfrak{u}(\mathfrak{g})$-modules

$$
0 \longrightarrow k \longrightarrow M \longrightarrow \Omega^{n-1}(k) \longrightarrow 0
$$

determined by $\zeta$. By [8, 6.3], $M$ is a $\mathfrak{u}(\mathfrak{g})$-module of constant Jordan type. We verify by inspection that the Jordan type of $M$ is $(a, 0, \ldots, 0,2)$ for some $a>0$ if $n$ is odd, and $(b, 1,0, \ldots, 0,1)$ for some $b>0$ if $n$ is even (see (3.1.2) for notation).

(2) Let $G=\mathbb{G}_{a(r)}$, and set $I$ equal to the augmentation ideal of $k G \simeq$ $k\left[u_{0}, \ldots, u_{p-1}\right] /\left(u_{0}^{p}, \ldots, u_{p-1}^{p}\right)$. As observed in [8], $I^{i} / I^{t}$ is a module of constant Jordan type for any $t>i$. As proven in [9], the only ideals of $k \mathbb{G}_{a(2)}$ which are of constant Jordan type are of the form $I^{i}$.

(3) As observed in $\left[8\right.$, the $n^{\text {th }}$ syzygy module $\Omega^{n}(k), n \in \mathbb{Z}$, is a module of constant Jordan type for any infinitesimal group scheme $G$. For $n$ even, $\Omega^{n}(k)$ has constant Jordan type $(a, 0, \ldots, 0,1)$ for some $a>0$, whereas for $n$ odd, $\Omega^{n}(k)$ has constant Jordan type $(b, p-1,0, \ldots, 0)$ for some $b>0$.

(4) For $G=\mathrm{SL}_{2(2)}$, we recall that the cohomology algebra $\mathrm{H}^{\bullet}(G, k)$ is generated modulo nilpotents by classes $\zeta_{1}, \zeta_{2}, \zeta_{3} \in \mathrm{H}^{2}(G, k)$ and classes $\xi_{1}, \xi_{2}, \xi_{3} \in \mathrm{H}^{2 p}(G, k)$ 
([19]). As in [8, 6.8], the $k G$-module

$$
M \equiv \operatorname{Ker}\left\{\sum \zeta_{i}+\sum \xi_{j}:\left(\Omega^{2}(k)\right)^{\oplus 3} \oplus\left(\Omega^{2 p}(k)\right)^{\oplus 3} \rightarrow k\right\}
$$

is a $k G$-module of constant Jordan type $(a, 0, \ldots, 0,1)$ for some $a>0$.

We elaborate on Example 5.6 (2), constructing $\mathbb{G}_{a(r)}$-modules of constant $j$-rank but not of constant Jordan type.

Example 5.7. We start with the following simple observation. Let $M_{1} \subset M_{2} \subset M$ be a chain of $k$-vector spaces, and let $\phi$ be an endomorphism of $M$ such that $\phi\left(M_{1}\right) \subset M_{1}$ and $\phi\left(M_{2}\right) \subset M_{2}$. If $\operatorname{dim}\left(\operatorname{Ker} \phi_{\left.\right|_{M_{1}}}\right)=\operatorname{dim}(\operatorname{Ker} \phi)$, then $\operatorname{dim}\left(\operatorname{Ker} \phi_{\left.\right|_{M_{1}}}\right)$ $=\operatorname{dim}\left(\operatorname{Ker} \phi_{\left.\right|_{M_{2}}}\right)=\operatorname{dim}(\operatorname{Ker} \phi)$.

Let $G=\mathbb{G}_{a(r)}$, and set $I$ equal to the augmentation ideal of

$$
k G \simeq k\left[u_{0}, \ldots, u_{p-1}\right] /\left(u_{0}^{p}, \ldots, u_{p-1}^{p}\right) .
$$

Consider any ideal $J$ of $k G$ with the property that $I^{i} \subset J$ for some $i, i \leq p-1$. Note that for any $\underline{a} \in \mathbb{A}^{r}$ and any $j \leq p-i$,

$$
\operatorname{dim}\left(\operatorname{Ker}\left\{\theta_{\underline{a}}^{j}: I^{i} \rightarrow I^{i}\right\}\right)=p j=\operatorname{dim}\left(\operatorname{Ker}\left\{\theta_{\underline{a}}^{j}: k G \rightarrow k G\right\}\right) .
$$

Indeed, since $I^{i}$ is a module of constant Jordan type, it suffices to check the statement for $\theta_{\underline{a}}=u_{0}$ for which it is straightforward. The observation in the previous paragraph together with (5.7.1) and the inclusions $I^{i} \subset J \subset k G$ imply

$$
\operatorname{dim}\left(\operatorname{Ker}\left\{\theta_{\underline{a}}^{j}: J \rightarrow J\right\}\right)=p j
$$

for any $j \leq p-i$ and any $\underline{a} \in \mathbb{A}^{r}$. Hence, $J$ has constant $j$-rank for $1 \leq j \leq p-i$.

In the following example, we offer a method applicable to almost all infinitesimal group schemes $G$ of constructing $k G$-modules which are of constant rank but not constant Jordan type.

Example 5.8. Let $G$ be an infinitesimal group scheme with the property that $V(G)$ has dimension at least 2. Assume that $p$ is odd, and let $n>0$ be an odd positive integer. Let $\zeta \in \mathrm{H}^{n}(G, k)$ be a non-zero cohomology class and let $M$ denote the kernel of $\zeta: \Omega^{n}(k) \rightarrow k$. Then $M$ has constant rank but not constant Jordan type. Namely, the local Jordan type of $M$ at $0 \neq v \in V(G)$ is $(a, 0,1,0, \ldots, 0)$ if $\zeta(v) \neq 0$ and is $(a-1,2,0, \ldots, 0)$ if $\zeta(v)=0$. These Jordan types have the same rank.

For $G=\mathrm{SL}_{2(1)}$, the restriction of any rational $\mathrm{SL}_{2}-$ module is a module of constant Jordan type (see [8]). Irreducible $\mathrm{SL}_{2}$-modules $S_{\lambda}$ are parameterized by their highest weight, a non-negative integer $\lambda$. Irreducible $\mathrm{SL}_{2(1)}$ modules are the restrictions of $S_{\lambda}$ to $\mathrm{SL}_{2(1)}$ for $0 \leq \lambda \leq p-1$.

Another important family of $\mathrm{SL}_{2}$-modules are the $V_{\lambda}$ (also denoted $H^{0}(\lambda)$ ) defined as the subspace of $k[s, t]$ (i.e., the symmetric algebra on the natural 2dimensional representation for $\mathrm{SL}_{2}$ ) consisting of homogeneous vectors of degree $\lambda$. For $0 \leq \lambda \leq p-1$, we have an isomorphism of $\mathrm{SL}_{2(1)}-$ modules: $S_{\lambda} \simeq V_{\lambda}$.

Recall that $V(G)$ is the nullcone in $s l_{2}$, and, hence,

$$
A=k[V(G)] \simeq k[x, y, z] /\left(x y+z^{2}\right) .
$$

Let

$$
i: \mathbb{P}^{1} \rightarrow \mathbb{P}(G)
$$


be the isomorphism given on homogeneous coordinates by

$$
\frac{k[x, y, z]}{\left(x y+z^{2}\right)} \rightarrow k[s, t], \quad(x, y, z) \mapsto\left(s^{2},-t^{2}, s t\right)
$$

In the next proposition, we compute explicitly the kernel bundles associated to the irreducible $\mathrm{SL}_{2(1)}$-modules and to the induced modules $V_{\lambda}$ for $p \leq \lambda \leq 2 p-2$. For convenience, we give the answer in terms of pull-backs to $\mathbb{P}^{1}$ via the isomorphism $i$.

Proposition 5.9. Let $G=\mathrm{SL}_{2(1)}$, and let $i: \mathbb{P}^{1} \stackrel{\sim}{\rightarrow} \mathbb{P}(G)$ be the isomorphism defined in (5.8.1).

(1) For $0 \leq \lambda \leq p-1$,

$$
i^{*}\left(\operatorname{Ker}\left\{\widetilde{\Theta}_{G}, S_{\lambda} \otimes \mathcal{O}_{\mathbb{P}(\mathrm{G})}\right\}\right) \simeq \mathcal{O}_{\mathbb{P}^{1}}(-\lambda) .
$$

(2) For $p \leq \lambda \leq 2 p-2$,

$$
i^{*}\left(\operatorname{Ker}\left\{\widetilde{\Theta}_{G}, V_{\lambda} \otimes \mathcal{O}_{\mathbb{P}(\mathrm{G})}\right\}\right) \simeq \mathcal{O}_{\mathbb{P}^{1}}(-\lambda) \oplus \mathcal{O}_{\mathbb{P}^{1}}(\lambda-2(p-1)) .
$$

Proof. We adopt the conventions of [2, §1]; in particular, we replace $\lambda$ by $m$. Let $v_{0}, v_{1}, \ldots, v_{m}$ be a basis for $V_{m}$ such that the generators $e, f$ and $h$ of $s l_{2}$ act as follows:

$$
\begin{aligned}
& h v_{i}=(2 i-m) v_{i}, \\
& f v_{i}=(m-i+1) v_{i-1} \text { for } i>0, f v_{0}=0, \\
& e v_{i}=(i+1) v_{i+1} \text { for } i<m, e v_{m}=0
\end{aligned}
$$

(see [21, 7.2] or [2, §1]). Recall that

$$
\Theta_{G}=x e+y f+z h
$$

(see Example 2.8(1)). Hence, the operator

$$
\Theta_{G}: V_{m} \otimes A \simeq A^{m+1} \longrightarrow V_{m} \otimes A \simeq A^{m+1}
$$

is represented by the matrix

$$
\left(\begin{array}{cccccc}
-m z & m y & 0 & \ldots & \ldots & \\
x & -(m-2) z & (m-1) y & 0 & \ldots & \\
0 & 2 x & -(m-4) z & (m-2) y & 0 & \ldots \\
0 & 0 & 3 x & -(m-6) z & (m-3) y & \ldots \\
\ldots & \ldots & \ldots & \ldots & \ldots & \\
\ldots & \ldots & \ldots & 0 & m x & m z
\end{array}\right)
$$

Substituting $\left(s^{2},-t^{2}, s t\right)$ for $(x, y, z)$, we get a degree two operator on $k[s, t]^{m+1}$ given by

$$
B_{m}(s, t)=\left(\begin{array}{cccccc}
-m s t & -m t^{2} & 0 & \ldots & \ldots & \\
s^{2} & (2-m) s t & (1-m) t^{2} & 0 & \ldots & \\
0 & 2 s^{2} & (4-m) s t & (2-m) t^{2} & 0 & \ldots \\
0 & 0 & 3 s^{2} & (6-m) s t & (3-m) t^{2} & \ldots \\
\ldots & \ldots & \ldots & \ldots & \ldots & \\
\ldots & \ldots & \ldots & 0 & m s^{2} & m s t
\end{array}\right) .
$$

One easily verifies that the vector

$$
w_{m}=\left[t^{m},-s t^{m-1}, s^{2} t^{m-2}, \ldots, \pm s^{m}\right]
$$


is annihilated by $B_{m}(s, t)$. For $0 \leq m \leq p-1$, the module $V_{m}$ is irreducible and the kernel bundle has rank 1 because the (constant) Jordan type of $V_{m}$ has a single block. The vector $w_{m}$ generates the kernel as a graded $k[s, t]$-module (no element of smaller degree lies in the kernel). Because $w_{m}$ is homogeneous of degree $m$ in $k[s, t]^{m+1}$, we conclude for $0 \leq m \leq p-1$ that

$$
i^{*}\left(\operatorname{Ker}\left\{\widetilde{\Theta}_{G}, V_{m} \otimes \mathcal{O}_{\mathbb{P}(\mathrm{G})}\right\}\right) \simeq \mathcal{O}_{\mathbb{P}^{1}}(-m), \quad 0 \leq m \leq p-1 .
$$

For $p \leq m \leq 2 p-2, V_{m}$ has a decomposition series which can be represented as follows:

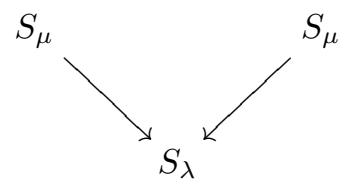

where $\lambda=2(p-1)-m, \mu=m-p=p-2-\lambda$, and $S_{\lambda}$, the irreducible module of highest weight $\lambda$, is the socle of $V_{m}([2, \S 1])$. By [8], $V_{m}$ has constant Jordan type. Plugging $x=1, y=z=0$ in (5.9.1), we get that the Jordan type is $[p]+[\mu+1]$. In particular, the rank of the kernel bundle is 2 .

Using the relations $\mu \equiv m(\bmod p)$ and $-\lambda \equiv \mu+2(\bmod p)$, we obtain that the matrix $B_{m}(s, t)$ has the following form:

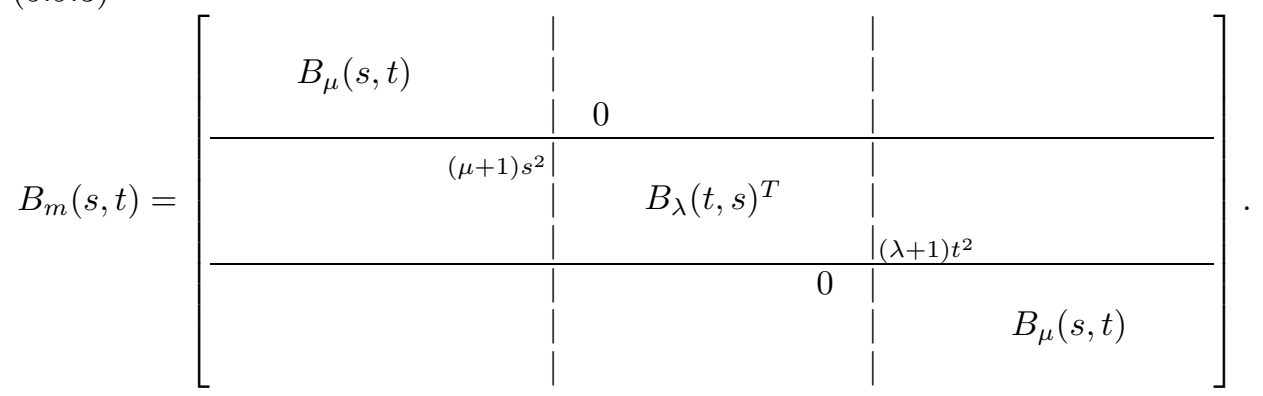

Here, the top left and bottom right corners are of size $\mu+1 \times \mu+1$, whereas the matrix in the center is of size $\lambda+1 \times \lambda+1$. The only non-zero entries outside of these three square diagonal blocks are at $(\mu+2, \mu+1)$ and $(\mu+\lambda+2, \mu+\lambda+3)$, equaled to $(\mu+1) s^{2}$ and $(\lambda+1) t^{2}$ respectively.

In particular, the $\mu+1 \times \lambda+1$ blocks above and below the matrix $B_{\lambda}(t, s)^{T}$ in the middle are zero. This implies that the kernel of this operator contains a copy of the kernel of $B_{\lambda}(s, t)$ (since it coincides with the kernel of $\left.B_{\lambda}(t, s)^{T}\right)$. We therefore obtain the vector

$$
w_{m}^{\prime}=\left[0, \ldots, 0, t^{\lambda},-s t^{\lambda-1}, \ldots, \mp s^{\lambda}, 0, \ldots, 0\right]
$$

in the kernel, where the non-zero entries are at the positions $(\mu+2, \ldots, \mu+\lambda+2)$.

One verifies that $\left\{w_{m}, w_{m}^{\prime}\right\}$ generate the kernel of $B_{m}(s, t)$ as a graded $k[s, t]$ module. For example, one can check this by restricting to the affine pieces $U(s \neq 0)$ and $U(t \neq 0)$. Hence,

$$
i^{*}\left(\operatorname{Ker}\left\{\widetilde{\Theta}_{G}, V_{m} \otimes \mathcal{O}_{\mathbb{P}(G)}\right\}\right) \simeq \mathcal{O}_{\mathbb{P}^{1}}(-m) \oplus \mathcal{O}_{\mathbb{P}^{1}}(m-2(p-1)) .
$$

One may readily determine the rank of various bundles of $\mathbb{P}(G)$ associated to modules of constant Jordan type using the next proposition. 
Proposition 5.10. Let $G$ be an infinitesimal group scheme, let $M$ be a $k G$-module of constant Jordan type $\sum_{i=1}^{p} a_{i}[i]$, and let $\mathcal{M}=M \otimes \mathcal{O}_{\mathbb{P}(\mathrm{G})}$. Then for any $j, 1 \leq$ $j<p$,

$$
\operatorname{rk}\left(\operatorname{Im}\left\{\widetilde{\Theta}_{G}^{j}, \mathcal{M}\right\}\right)=\sum_{i=j+1}^{p} a_{i}(i-j)
$$

In particular,

$$
\operatorname{Ker}\left\{\widetilde{\Theta}_{G}, \mathcal{M}\right\} \subset \operatorname{Ker}\left\{\widetilde{\Theta}_{G}^{2}, \mathcal{M}\right\} \subset \cdots \subset \operatorname{Ker}\left\{\widetilde{\Theta}_{G}^{p-1}, \mathcal{M}\right\} \subset \mathcal{M}
$$

is a chain of $\mathcal{O}_{\mathbb{P}(\mathrm{G})}$-submodules with $\operatorname{rk}\left(\operatorname{Ker}\left\{\widetilde{\Theta}_{G}^{j-1}, \mathcal{M}\right\}\right)<\operatorname{rk}\left(\operatorname{Ker}\left\{\widetilde{\Theta}_{G}^{j}, \mathcal{M}\right\}\right)$ if and only if $a_{i} \neq 0$ for some $1 \leq j \leq i \leq p$.

Proof. The formula (5.10.1) is the formula for the rank of $u^{j}$ on the $k[u] / u^{p}$-module $\bigoplus_{i}\left(k[u] / u^{i}\right)^{\oplus a_{i}}$ of Jordan type $\sum_{i=1}^{p} a_{i}[i]$. This is therefore the dimension of the image of $\theta_{v}, 0 \neq v \in V(G)$ on $M_{k(v)}$, and thus the rank of the vector bundle $\operatorname{Im}\left\{\widetilde{\Theta}_{G}^{j}, \mathcal{M}\right\}$ by Theorem 4.13 .

The following class of modules, of interest in its own right, is currently being studied by Jon Carlson and the authors.

Definition 5.11. Let $G$ be an infinitesimal group scheme, $M$ a finite dimensional $k G$-module, and $j<p$ a positive integer. We say that a $k G$-module $M$ has the constant $j$-image property if there exists a subspace $I(j) \subset M$ such that for every $v \neq 0$ in $V(G)$, the image of $\theta_{v}^{j}: M_{k(v)} \rightarrow M_{k(v)}$ equals $I(j)_{k(v)}$. Similarly, we say that $M$ has constant $j$-kernel property if there exists some submodule $K(j) \subset M$ such that for every $v \neq 0$ in $V(G)$, the kernel of $\theta_{v}^{j}: M_{k(v)} \rightarrow M_{k(v)}$ equals $K(j)_{k(v)}$.

We see that these modules are precisely those whose associated vector bundles are trivial vector bundles.

Proposition 5.12. Let $G$ be an infinitesimal group scheme, and let $M$ be a $k G$ module of constant $j$-rank. Then the algebraic vector bundle $\operatorname{Im}\left\{\widetilde{\Theta}_{G}^{j}, \mathcal{M}\right\}$ is trivial (i.e., a free coherent sheaf) on $\mathbb{P}(\mathrm{G})$ if and only if $M$ has the constant $j$-image property. Similarly, $\operatorname{Ker}\left\{\widetilde{\Theta}_{G}^{j}, \mathcal{M}\right\}$ is trivial if and only if $M$ has the constant $j$ kernel property.

Proof. If $M$ has a constant $j$-image property, then $\operatorname{Im}\left\{\widetilde{\Theta}_{G}^{j}, \mathcal{M}\right\}$ is a free $\mathcal{O}_{X}$-module generated by $I(j)$. Conversely, assume that $\operatorname{Im}\left\{\widetilde{\Theta}_{G}^{j}, \mathcal{M}\right\}$ is a free $\mathcal{O}_{X}$-module. Then there exists a subspace $I(j) \subset M=\Gamma(X, \mathcal{M})$ which maps to and spans each fiber $\operatorname{Im}\left\{\theta_{v}^{j}, M_{k(v)}\right\}$, for $0 \neq v \in V(G)$. The argument for kernels is similar.

Remark 5.13. We point out that the properties of constant $j$-image and constant $j$-kernel are independent of each other. Consider the module $M^{\#}$ of Example 6.1 As shown in that example, $\operatorname{Ker}\left\{\widetilde{\Theta}_{G}, \mathcal{M}^{\#}\right\}$ is locally free of rank 2 but not free, since the global sections have dimension one. On the other hand, $\operatorname{Im}\left\{\widetilde{\Theta}_{G}, \mathcal{M}^{\#}\right\}$ is a free $\mathcal{O}_{X}$-module generated by the global section $n_{3}$. In particular, $M^{\#}$ has constant 1-image property but not constant 1-kernel property.

For the module $M$ of Example 6.1, the sheaf $\operatorname{Ker}\left\{\widetilde{\Theta}_{G}, \mathcal{M}\right\}$ is free of rank 2, whereas $\operatorname{Im}\left\{\widetilde{\Theta}_{G}, \mathcal{M}\right\}$ is locally free of rank 1 but not free since it does not have any global sections. Hence, $M$ has a constant 1-kernel property but not constant 1-image property. 
We consider an analogue of the sheaf construction of Duflo-Serganova for Lie superalgebras [11. This construction enables us to produce additional algebraic vector bundles on $\mathbb{P}(\mathrm{G})$. We implicitly use the observation $\widetilde{\Theta}_{G}^{p}=0$.

Definition 5.14. Let $G$ be an infinitesimal group scheme, and let $M$ be a finite dimensional $k G$-module. Let $\mathcal{M}=M \otimes \mathcal{O}_{\mathbb{P}(\mathrm{G})}$. For any $i, 1 \leq i \leq p-1$, we define coherent $\mathcal{O}_{\mathbb{P}(\mathrm{G})}$-modules, subquotients of $\mathcal{M}$ :

$$
\mathcal{M}^{[i]} \equiv \operatorname{Ker}\left\{\widetilde{\Theta}_{G}^{i}, \mathcal{M}\right\} / \operatorname{Im}\left\{\widetilde{\Theta}_{G}^{p-i}, \mathcal{M}\right\} .
$$

The following simple lemma helps to motivate these subquotients.

Lemma 5.15. Let $V$ be a finite dimensional $k[t] / t^{p}$-module, and let $\operatorname{JType}(V, t)=$ $\left(a_{p}, \ldots, a_{1}\right)$ (using the notation introduced in (3.1.2)). Let

$$
V^{[j]}=\operatorname{Ker}\left\{t^{j}: V \rightarrow V\right\} / \operatorname{Im}\left\{t^{p-j}: V \rightarrow V\right\}
$$

for $j \leq p-1$. Then

$$
\operatorname{dim}\left(V^{[j]}\right)=\sum_{1 \leq i \leq j} i a_{i}+\sum_{i>j} j a_{i}-\sum_{i+j>p}(i+j-p) a_{i} .
$$

In particular, $V$ is projective as a $k[t] / t^{p}$-module if and only if $V^{[1]}=0$. Furthermore, for $j \leq p-1, V^{[j]} \simeq V^{[p-j]}$ as $k[t] / t^{p}$-modules.

As seen in the next proposition, these subquotients can provide additional examples of algebraic vector bundles over $\mathbb{P}(\mathrm{G})$.

Proposition 5.16. Let $G$ be an infinitesimal group scheme and let $M$ be a finite dimensional $k G$-module which is of constant $j$-rank and constant $(p-j)$-rank for some $j, 1 \leq j<p$. Then $\mathcal{M}^{[j]}$ is a locally free $\mathcal{O}_{X}$-module and $\mathcal{M}^{[j]} \otimes_{\mathcal{O}_{X}} k(x) \rightarrow$ $M_{k(x)}^{[j]}$ is an isomorphism for all $x \in X \equiv \mathbb{P}(\mathrm{G})$.

Proof. For any $x \in X$, consider the map of exact sequences

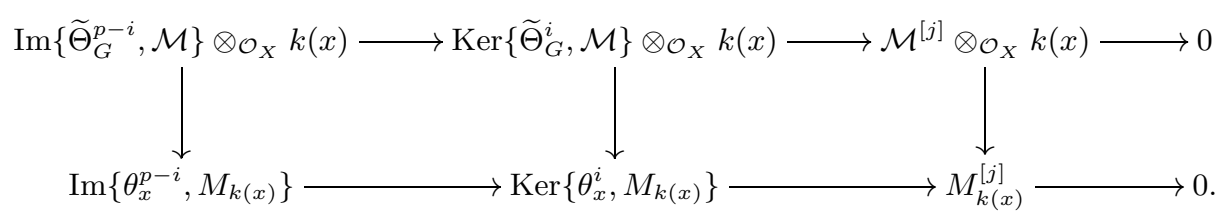

The left and middle vertical maps are isomorphisms by Theorem 4.13 Thus, the 5-lemma implies that the right vertical arrow is also an isomorphism.

We give an application of this $(-)^{[1]}$ construction to endotrivial modules. An interested reader can compare our construction to [1. Recall that a module $M$ of a finite group scheme $G$ is endotrivial if $\operatorname{End}_{k}(M) \simeq k+$ proj. It was shown in [8, $\S 5]$ that an endotrivial module is a module of constant Jordan type with possible types $[1]+$ proj and $[p-1]+$ proj.

Proposition 5.17. Let $G$ be an infinitesimal group scheme, and assume that $G$ has a subgroup scheme isomorphic to $\mathbb{G}_{a(1)} \times \mathbb{G}_{a(1)}$ or $\mathbb{G}_{a(2)}$. Let $M$ be a module of constant Jordan type, and set $\mathcal{M}=M \otimes \mathcal{O}_{\mathbb{P}(G)}$. Then $\mathcal{M}^{[1]}$ is a line bundle (i.e., an algebraic vector bundle of rank one) if and only if $M$ is endotrivial. 
Proof. The sheaf $\mathcal{M}^{[1]}$ is locally free by Lemma 5.15, Let $\sum_{i=1}^{p} a_{i}[i]$ be the Jordan type of $M$. Lemma 5.15 implies that the rank of the vector bundle $\mathcal{M}^{[1]}$ equals $\sum_{i=0}^{p-1} a_{i}$. Hence, $\mathcal{M}^{[1]}$ is a line bundle if and only if the Jordan type of $M$ has only one non-projective block. A theorem of D. Benson 4 states that modules of constant Jordan type with unique non-projective block must be of type [1] + proj or $[p-1]+$ proj. By [8, $\S 5]$, this happens if and only if $M$ is endotrivial.

We next give a global version of the observation in Lemma 5.15 that $V^{[j]} \simeq V^{[p-j]}$ for $j \leq p-1$. Recall that for a variety $X$ and a coherent sheaf $\mathcal{E}$, we denote by $\mathcal{E}^{\vee}=\operatorname{Hom}_{\mathcal{O}_{X}}\left(\mathcal{E}, \mathcal{O}_{X}\right)$ the dual sheaf.

Proposition 5.18. Let $G$ be an infinitesimal group scheme, and let $M$ be a $k G$ module which is of constant $j$-rank and of constant $(p-j)$-rank for some $j, 1 \leq$ $j<p$. Let $\mathcal{M}=M \otimes \mathcal{O}_{\mathbb{P}(\mathrm{G})}, \mathcal{N}=M^{\#} \otimes \mathcal{O}_{\mathbb{P}(\mathrm{G})}$. Then

$$
\mathcal{N}^{[p-j]} \simeq\left(\mathcal{M}^{[j]}\right)^{\vee}
$$

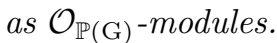

Proof. Let $X=\mathbb{P}(\mathrm{G})$. As discussed in the proof of Proposition 5.5, the $\mathcal{O}_{X}$-linear dual of the complex of $\mathcal{O}_{X}$-modules

$$
\mathcal{M}\left(-(p-j) p^{r-1}\right) \stackrel{\widetilde{\Theta}_{G}^{p-j}\left(-(p-j) p^{r-1}\right)}{\longrightarrow} \mathcal{M} \stackrel{\widetilde{\Theta}_{G}^{j}}{\longrightarrow} \mathcal{M}\left(j p^{r-1}\right)
$$

is the complex

$$
\mathcal{N}\left((p-j) p^{r-1}\right) \stackrel{\widetilde{\Theta}_{G}^{p-j}}{\longleftarrow} \mathcal{N} \stackrel{\widetilde{\Theta}_{G}^{j}\left(-j p^{r-1}\right)}{\longleftarrow} \mathcal{N}\left(-j p^{r-1}\right) .
$$

A similar statement applies with $\theta_{v}$ in place of $\widetilde{\Theta}_{G}$.

For any scheme $Y$ and any complex of $\mathcal{O}_{Y}$-modules

$$
S_{1} \stackrel{f}{\longrightarrow} S_{2} \stackrel{g}{\longrightarrow} S_{3}
$$

with $\mathcal{O}_{Y}$-linear dual

$$
S_{1}^{\vee} \stackrel{f^{\vee}}{\longleftarrow} S_{2}^{\vee} \stackrel{g^{\vee}}{\longleftarrow} S_{3}^{\vee}
$$

there is a natural pairing

$$
(\operatorname{Ker}\{g\} / \operatorname{Im}\{f\}) \otimes\left(\operatorname{Ker}\left\{f^{\vee}\right\} / \operatorname{Im}\left\{g^{\vee}\right\}\right) \longrightarrow \mathcal{O}_{Y},
$$

induced by the evident pairing

$$
S_{2} \otimes S_{2}^{\vee} \rightarrow \mathcal{O}_{Y}
$$

In particular, we have a pairing

$$
\mathcal{M}^{[j]} \otimes \mathcal{N}^{[p-j]} \rightarrow \mathcal{O}_{X}
$$

and, hence, a map

By Proposition 5.16 .

$$
f: \mathcal{N}^{[p-j]} \rightarrow\left(\mathcal{M}^{[j]}\right)^{\vee}
$$

$$
\mathcal{M}^{[j]} \otimes_{\mathcal{O}_{X}} k(x) \simeq M_{k(x)}^{[j]}, \quad \mathcal{N}^{[p-j]} \otimes_{\mathcal{O}_{X}} k(x) \simeq N_{k(x)}^{[p-j]}
$$


for any $x \in X$. By naturality, the specialization of $f$ at a point $x$ corresponds to the map $f_{x}: N_{k(x)}^{[p-j]} \rightarrow\left(M_{k(x)}^{[j]}\right)$ \# induced by the pairing (5.18.1) for $Y=\operatorname{Spec} k(x)$. One readily verifies that this is a perfect pairing if $Y=\operatorname{Spec} k(x)$. Hence,

$$
f \otimes_{O_{X}} k(x): \mathcal{N}^{[p-j]} \otimes_{\mathcal{O}_{X}} k(x) \rightarrow\left(\mathcal{M}^{[j]}\right)^{\vee} \otimes_{\mathcal{O}_{X}} k(x)
$$

is an isomorphism for any $x \in X$. Therefore, $\mathcal{N}^{[p-j]} \simeq\left(\mathcal{M}^{[j]}\right)^{\vee}$.

Consideration of $\mathcal{M}^{[1]}$ leads to another characterization of projective $k G$-modules.

Proposition 5.19. Let $G$ be an infinitesimal group scheme and let $M$ be a finite dimensional $k G$-module. Then $M$ is projective if and only if $M$ has constant rank, has constant $(p-1)$-rank, and satisfies $\mathcal{M}^{[1]}=0$.

Proof. Assume that $M$ is a projective $k G$-module. Then $M$ has constant Jordan type (which is some multiple of $[p])$ and hence has constant rank and constant $(p-$ 1 )-rank. For any $x \in \mathbb{P}(\mathrm{G})=X, \theta_{x}^{*}\left(M_{k(x)}\right)$ is a free $k(x)[t] / t^{p}$-module of rank equal to $\frac{\operatorname{dim}(M)}{p}$. If we lift a basis of this free module to $\mathcal{M}_{(x)} \equiv \mathcal{M} \otimes_{\mathcal{O}_{X}} \mathcal{O}_{X, x}$, then an application of Nakayama's Lemma tells us that $\mathcal{M}_{(x)}$ is free as an $\mathcal{O}_{X, x}[t] / t^{p}$-module. This readily implies that $\left(\mathcal{M}_{(x)}\right)^{[p-1]} \equiv \operatorname{Ker}\left\{\widetilde{\Theta}_{G,(x)}^{p-1}, \mathcal{M}_{(x)}\right\} / \operatorname{Im}\left\{\widetilde{\Theta}_{G,(x)}, \mathcal{M}_{(x)}\right\}$ vanishes. Using the exactness of localization, we conclude that

$$
\left(\mathcal{M}^{[p-1]}\right)_{(x)}=\left(\mathcal{M}_{(x)}\right)^{[p-1]} .
$$

Consequently, $\mathcal{M}^{[p-1]}=0$. By Proposition 5.18 , we conclude that $\mathcal{M}^{[1]}=0$.

Conversely, if $M$ has constant rank and constant $(p-1)$-rank and if $\mathcal{M}^{[1]}=0$, then Proposition 5.16 tells us that $M_{k(x)}^{[1]} \equiv \operatorname{Ker} \theta_{x} / \operatorname{Im} \theta_{x}^{p-1}$ equals 0 for all $x \in X$. Lemma 5.15 thus implies that each $M_{k(x)}$ is projective, so that the local criterion for projectivity [25] implies that $M$ is projective.

One very simple invariant of the algebraic vector bundle $\operatorname{Ker}\left\{\widetilde{\Theta}_{G}^{j}, \mathcal{M}\right\}$ is the dimension of its vector space of global sections. The following proposition gives some understanding of $\Gamma\left(\mathbb{P}(\mathrm{G}), \operatorname{Ker}\left\{\widetilde{\Theta}_{G}^{j}, \mathcal{M}\right\}\right) \subset \Gamma(\mathbb{P}(\mathrm{G}), \mathcal{M})$.

Proposition 5.20. Let $G$ be an infinitesimal group scheme, and assume that $V(G)$ is reduced. Let $M$ be a $k G$-module and let $\mathcal{M}=M \otimes \mathcal{O}_{\mathbb{P}(\mathrm{G})}$. Then

$$
\Gamma\left(\mathbb{P}(\mathrm{G}), \operatorname{Ker}\left\{\widetilde{\Theta}_{G}^{j}, \mathcal{M}\right\}\right) \subset M
$$

consists of those $m \in M$ such that $\theta_{x}^{j}(m)=0$ for all $x \in \mathbb{P}(\mathrm{G})$.

Proof. Recall that $\mathbb{P}(\mathrm{G})$ is connected by $[8,3.4]$, and thus $\Gamma(\mathbb{P}(\mathrm{G}), \mathcal{M})=M$. Under this identification, the global sections of $\operatorname{Ker}\left\{\widetilde{\Theta}_{G}^{j}, \mathcal{M}\right\}$ coincide with the subset

$$
\left\{m \in M \mid \Theta_{G}^{j}(m \otimes 1)=0\right\} .
$$

Since $V(G)$ is reduced, we have $\Theta_{G}^{j}(m \otimes 1)=0$ if and only if

$$
\theta_{v}^{j}(m \otimes 1)=\Theta_{G}^{j}(m \otimes 1) \otimes_{k[V(G)]} k(v)=0
$$

for every $v \in V(G)$. Hence, $m \in \Gamma\left(\mathbb{P}(\mathrm{G}), \operatorname{Ker}\left\{\widetilde{\Theta}_{G}^{j}, \mathcal{M}\right\}\right)$ if and only if $m \in$ $\operatorname{Ker}\left\{\theta_{v}^{j}, M_{k(v)}\right\}$ for every $v \in V(G)$ if and only if $\theta_{x}^{j}(m)=0$ for all $x \in \mathbb{P}(\mathrm{G})$.

We make Proposition 5.20 more explicit in the case of a classical Lie algebra. 
Proposition 5.21. Let $\mathfrak{G}$ be a (reduced, irreducible) algebraic group over $k$, let $G=\mathfrak{G}_{(1)}$, and let $\mathfrak{g}=\operatorname{Lie}(\mathfrak{G})$. Assume that $\mathfrak{g}$ is generated by p-nilpotent elements.

(1) If $M$ is a rational $\mathfrak{G}$-module, then $\Gamma\left(\mathbb{P}(G), \operatorname{Ker}\left\{\widetilde{\Theta}_{G}^{j}, \mathcal{M}\right\}\right)$ is a rational $\mathfrak{G}$ submodule of $M$.

(2) If $V(G)$ is reduced, then

$$
\Gamma\left(\mathbb{P}(\mathrm{G}), \operatorname{Ker}\left\{\widetilde{\Theta}_{G}, \mathcal{M}\right\}\right)=\mathrm{H}^{0}(G, M) .
$$

Proof. To prove that $\Gamma\left(\mathbb{P}(\mathrm{G}), \operatorname{Ker}\left\{\widetilde{\Theta}_{G}^{j}, \mathcal{M}\right\}\right)$ is a $\mathfrak{G}$-submodule of $M$, we may base change to the algebraic closure of $k$, and thus we may assume $k$ is algebraically closed. Let $g \in \mathfrak{G}$ be a $k$-rational point. Then

$$
\theta_{v}^{j}(g m \otimes 1)=g \theta_{v^{g^{-1}}}^{j}(m \otimes 1),
$$

where the action of $\mathfrak{G}$ on $V(G)=\mathcal{N}_{p}(\mathfrak{g})$ (the $p$-nilpotent cone of $\mathfrak{g}$ ) is via the adjoint action of $\mathfrak{G}$.

Hence, we have the following equalities:

$$
\begin{aligned}
\{m & \left.\in M \mid \Theta_{G}^{j}(m \otimes 1)=0\right\}=\bigcap_{0 \neq v \in V(G)}\left\{m \in M \mid \theta_{v}^{j}(m \otimes 1)=0\right\} \\
& =\bigcap_{0 \neq \operatorname{Ad}\left(g^{-1}\right) v \in V(G)}\left\{m \in M \mid g \theta_{\operatorname{Ad}\left(g^{-1}\right) v}^{j}(m \otimes 1)=0\right\} \\
& =\bigcap_{0 \neq v \in V(G)}\left\{m \in M \mid \theta_{v}^{j}(g m \otimes 1)=0\right\}=\left\{m \in M \mid \Theta_{G}^{j}(g m \otimes 1)=0\right\},
\end{aligned}
$$

where the first and the last equality follow from Proposition 5.20 , the second equality follows from the fact that $\operatorname{Ad}\left(g^{-1}\right): V(G) \rightarrow V(G)$ is a bijection, and the third equality from (5.21.1). We conclude that $\left\{m \in M \mid \Theta_{G}^{j}(m \otimes 1)=0\right\}$ is a $\mathfrak{G}$-stable subspace of $M$.

The second assertion follows immediately from Proposition 5.20 and the fact that $v \in V(G)$ corresponds to a $p$-nilpotent element $X_{v}$ of $\mathfrak{g}$ and that the action of $\theta_{v}$ is the action of $X_{v}$.

Combining Proposition 5.10 and Proposition 5.21 in the special case $j=1$ yields the following criterion for the non-triviality of $\operatorname{Ker}\left\{\widetilde{\Theta}_{G}, \mathcal{M}\right\}$.

Corollary 5.22. Let $G$ be an infinitesimal group scheme such that $V(G)$ is reduced and positive dimensional. Assume that for any field extension $K / k, K G$ is generated by $\theta_{v} \in k(v) G$, for all $v \in V(G)$ such that $k(v) \subset K$. Let $M$ be a finite dimensional $k G$-module of constant Jordan type $\sum_{i} a_{i}[i]$. If

$$
\operatorname{dim} \mathrm{H}^{0}(G, M)<\sum_{i=1}^{p} a_{i},
$$

then $\operatorname{Ker}\left\{\widetilde{\Theta}_{G}, \mathcal{M}\right\}$ is a non-trivial algebraic vector bundle over $\mathbb{P}(\mathrm{G})$.

Proof. By Proposition 5.10, the dimension of the fibers of $\operatorname{Ker}\left\{\widetilde{\Theta}_{G}, \mathcal{M}\right\}$ is $\operatorname{dim} M-$ $\sum_{i=2}^{p} a_{i}(i-1)=\sum_{i=1}^{p} a_{i}$. By Proposition 5.20 the global sections of $\operatorname{Ker}\left\{\widetilde{\Theta}_{G}, \mathcal{M}\right\}$ equal $\mathrm{H}^{0}(G, M)$. Hence, the inequality $\operatorname{dim} \mathrm{H}^{0}(G, M)<\sum_{i=1}^{p} a_{i}$ implies that the dimension of the global sections is less than the dimension of the fibers. Therefore, the sheaf is not free.

The following two lemmas will be applied to prove Proposition 5.25 . 
Lemma 5.23. Let $R$ be a local commutative ring with residue field $k$ and let $M$ be a finite $R[t] / t^{p}$-module which is free as an $R$-module. If $M \otimes_{R} k$ is a free $k[t] / t^{p}$ module, then $M$ is free as an $R[t] / t^{p}$-module.

Proof. Let $m_{1}, \ldots, m_{s} \in M$ be such that $\bar{m}_{1}, \ldots, \bar{m}_{s}$ form a basis for $M \otimes_{R} k$ as a $k[t] / t^{p}$-module. Let $Q$ be a free $R[t] / t^{p}$-module of rank $s$ with basis $q_{1}, \ldots, q_{s}$ and consider the $R[t] / t^{p}$-module homomorphism $f: Q \rightarrow M$ sending $q_{i}$ to $m_{i}$.

By Nakayama's Lemma, $f: Q \rightarrow M$ is surjective. Because $M$ is free as an $R$ module, applying $-\otimes_{R} k$ to the short exact sequence $0 \rightarrow \operatorname{Ker}\{f\} \rightarrow Q \rightarrow M \rightarrow 0$ determines the short exact sequence

$$
0 \rightarrow \operatorname{Ker}\{f\} \otimes_{R} k \rightarrow Q \otimes_{R} k \rightarrow M \otimes_{R} k \rightarrow 0 .
$$

Consequently, $\operatorname{Ker}\{f\} \otimes_{R} k=0$, so that another application of Nakayama's Lemma implies that $\operatorname{Ker}\{f\}=0$. Hence, $f$ is an isomorphism, and thus $M$ is free as an $R[t] / t^{p}$-module.

Lemma 5.24. Let $G$ be an infinitesimal group scheme and $M$ be a finite dimensional $k G$-module. Set $A=k[V(G)]$; for any $f \in A$, set $A_{f}=A[1 / f]$. Assume that Spec $A_{f} \subset V(G)$ has empty intersection with $V(G)_{M}$. Then $\left(\mathcal{U}_{G} \circ \epsilon\right)^{*}\left(M \otimes A_{f}\right)$ is a projective $A_{f}[t] / t^{p}$-module.

Proof. By definition, $V(G)_{M}$ consists of those points $v \in V(G)$ such that $\theta_{v}^{*}\left(M_{(k(v)}\right)$ is not free as a $k(v)[t] / t^{p}$-module. By the universal property of $\mathcal{U}_{G} \circ \epsilon$, the assumption that Spec $A_{f} \cap V(G)_{M}=\emptyset$ implies for every point $v \in \operatorname{Spec} A_{f}$ that $\theta_{v}^{*}\left(M_{k(v)}\right)=\left(\mathcal{U}_{G} \circ \epsilon\right)^{*}\left(M \otimes A_{f}\right) \otimes_{A_{f}} k(v)$ is free as a $k(v)[t] / t^{p}$-module. Let $A_{(v)}$ denote the localization of $A$ at $v$. Then Lemma 5.23 implies for every point $v \in \operatorname{Spec} A_{f}$ that the localization $\left(\mathcal{U}_{G} \circ \epsilon\right)^{*}\left(M \otimes A_{f}\right) \otimes_{A_{f}} A_{(v)}$ is free as a $A_{(v)}[t] / t^{p}-$ module. This implies that $M \otimes A_{f}$ is projective (since projectivity of a finitely generated module over a $A$ is determined locally).

We conclude with a property of the (projectivized) rank variety $\mathbb{P}(\mathrm{G})_{M}$ of a $k G$-module $M$.

Proposition 5.25. Let $G$ be an infinitesimal group scheme, $M$ be a finite dimensional $k G$-module, and set $\mathcal{M}=M \otimes \mathcal{O}_{\mathbb{P}(\mathrm{G})}$. Then

$$
\operatorname{Supp}_{\mathcal{O}_{\mathbb{P}(\mathrm{G})}}\left(\mathcal{M}^{[1]}\right) \subset \mathbb{P}(\mathrm{G})_{M},
$$

where $\operatorname{Supp}_{\mathcal{O}_{\mathbb{P}(\mathrm{G})}}\left(\mathcal{M}^{[1]}\right)$ is the support of the coherent sheaf $\mathcal{M}^{[1]}$ (the closed subset of points $x \in \mathbb{P}(\mathrm{G})$ at which $\left.\mathcal{M}_{(x)}^{[1]} \neq 0\right)$.

Proof. Let $A$ denote $k[V(G)]$ and let $X$ denote $\mathbb{P}(\mathrm{G})$. Consider some $x \notin X_{M}$ and choose some homogeneous polynomial $F \in A$ vanishing on $X_{M}$ such that $F(x) \neq 0$. Thus, $x \in \operatorname{Spec}\left(A_{F}\right)_{0} \subset X$ and $\operatorname{Spec}\left(A_{F}\right)_{0} \cap X_{M}=\emptyset$, where $\left(A_{F}\right)_{0}$ denotes the elements of degree 0 in the localization $A_{F}=A[1 / F]$. It suffices to prove that $x \notin \operatorname{Supp}_{\mathcal{O}_{X}}\left(\mathcal{M}^{[1]}\right) \cap \operatorname{Spec}\left(A_{F}\right)_{0}$. Since localization is exact, this is equivalent to proving that $v \notin \operatorname{Supp}_{A_{F}}\left(\left(M \otimes A_{F}\right)^{[1]}\right)$ for some $v \in \operatorname{Spec} A_{F}$ mapping to $x$.

The condition $\operatorname{Spec}\left(A_{F}\right)_{0} \cap X_{M}=\emptyset$ implies that $\operatorname{Spec}\left(A_{F}\right) \cap V(G)_{M}=\emptyset$. Hence, by Lemma [5.24. $\left(\mathcal{U}_{G} \circ \epsilon\right)^{*}\left(M \otimes A_{F}\right)$ is a projective $A_{F}[t] / t^{p}$-module. This implies that $\left(\mathcal{U}_{G} \circ \epsilon\right)^{*}\left(\left(M \otimes A_{F}\right)^{[1]}\right)=0$ and thus that $v \notin \operatorname{Supp}_{A_{F}}\left(\left(M \otimes A_{F}\right)^{[1]}\right)$. 
Remark 5.26. The reverse inclusion $\mathbb{P}(\mathrm{G})_{M} \subset \operatorname{Supp}_{\mathcal{O}_{\mathbb{P}(\mathrm{G})}}\left(\mathcal{M}^{[1]}\right)$ seems closely related to the condition that $\operatorname{Ker}\left\{\widetilde{\Theta}_{G}, \mathcal{M}\right\} \otimes_{\mathcal{O}_{\mathbb{P}(\mathrm{G})}} k(x) \rightarrow \operatorname{Ker}\left\{\theta_{x}, M_{k(x)}\right\}$ be surjective.

\section{EXAMPLES AND CALCULATiONS With BUNDLES}

In this final section, we investigate numerous specific examples. The case in which $G$ equals either $\mathbb{G}_{a(1)}^{\times 2}$ or $\underline{s}_{2}$ (the infinitesimal group scheme associated to the restricted Lie algebra $s l_{2}$ ) is particularly amenable to computation for $\mathbb{P}(G)$ is isomorphic to $\mathbb{P}^{1}$. Specifically, we do calculations for projective $k G$-modules, examples of modules of constant Jordan type which are not distinguished by support varieties. We also compute bundles obtained from "zig-zag modules" and syzygies.

As we see in the following simple example, the isomorphism type of the vector bundles discussed in Theorem 5.1 can be used to distinguish certain $k G$-modules which have the same local Jordan type. We remind the reader that the local Jordan type of a finite dimensional $k G$-module $M$ of constant Jordan type is the same as that of its linear dual $M^{\#}$.

Example 6.1. Let $G=\mathbb{G}_{a(2)}$ so that $k \mathbb{G}_{a(2)}=k\left[u_{0}, u_{1}\right] /\left(u_{0}^{p}, u_{1}^{p}\right), V\left(\mathbb{G}_{a(2)}\right)=\mathbb{A}^{2}$, $A=k\left[V\left(\mathbb{G}_{a(2)}\right)\right]=k\left[x_{0}, x_{1}\right]$ graded so that $x_{0}$ is given degree 1 and $x_{1}$ is given degree $p$. Then

$$
\Theta_{G}=x_{1} u_{0}+x_{0}^{p} u_{1} \in A\left[u_{0}, u_{1}\right] /\left(u_{0}^{p}, u_{1}^{p}\right)
$$

(see Example 2.6(2)). We consider the 3-dimensional $k G$-module $M$ of constant Jordan type $[2]+[1]$ and its linear dual $M^{\#}$, which we represent diagrammatically as follows:
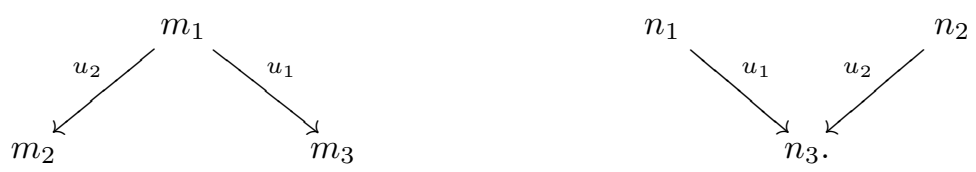

The $k \mathbb{G}_{\mathrm{a}(2)}$-invariant subspace of $M$ is two dimensional, and, hence, the global sections of $\operatorname{Ker}\left\{\widetilde{\Theta}_{G}, M \otimes \mathcal{O}_{\mathbb{P}(G)}\right\}$ have dimension two by Proposition 5.20 (in fact, an explicit calculation shows that this is a trivial bundle of rank 2). On the other hand, $M^{\#}$ has only 1-dimensional invariant subspace and, hence, the global sections of $\operatorname{Ker}\left\{\widetilde{\Theta}_{G}, M^{\#} \otimes \mathcal{O}_{\mathbb{P}(G)}\right\}$ have dimension one. Thus,

$$
\operatorname{Ker}\left\{\widetilde{\Theta}_{G}, M \otimes \mathcal{O}_{\mathbb{P}(G)}\right\} \nsubseteq \operatorname{Ker}\left\{\widetilde{\Theta}_{G}, M^{\#} \otimes \mathcal{O}_{\mathbb{P}(G)}\right\} .
$$

We next give a somewhat more interesting example of pairs of modules of the same constant Jordan type with different associated bundles.

Example 6.2. As in Proposition 5.9, let $S_{\lambda}$ be the irreducible $\mathrm{SL}_{2}$-module of highest weight $\lambda, 0 \leq \lambda \leq p-1$, and consider $S^{p}\left(S_{\lambda}\right)$, the $p^{\text {th }}$ symmetric power of $S_{\lambda}$. Since $S_{\lambda}$ is self-dual, the dual of $S^{p}\left(S_{\lambda}\right)$ is $\Gamma^{p}\left(S_{\lambda}\right)$, the $p^{\text {th }}$ divided power of $S_{\lambda}$. We have a short exact sequence of rational $\mathrm{SL}_{2}$-modules:

$$
0 \longrightarrow S_{\lambda}^{(1)} \longrightarrow S^{p}\left(S_{\lambda}\right) \longrightarrow \Gamma^{p}\left(S_{\lambda}\right) \longrightarrow S_{\lambda}^{(1)} \longrightarrow 0 .
$$

Here, $S_{\lambda}^{(1)}$ is the first Frobenius twist of $S_{\lambda}$, and thus trivial as a $\mathfrak{u}\left(s l_{2}\right)$-module.

Let $X=\operatorname{Proj} k\left[N\left(s l_{2}\right)\right]$. By Proposition [5.20, the space of global sections of $\operatorname{Ker}\left\{\widetilde{\Theta}_{\underline{s} l_{2}}, S^{p}\left(S_{\lambda}\right) \otimes \mathcal{O}_{X}\right\}$ equals the $s l_{2}$ invariants of $S^{p}\left(S_{\lambda}\right)$. Hence,

$$
\Gamma\left(X, \operatorname{Ker}\left\{\widetilde{\Theta}_{\underline{s} l_{2}}, S^{p}\left(S_{\lambda}\right) \otimes \mathcal{O}_{X}\right\}\right)=S_{\lambda}^{(1)} .
$$


On the other hand, $\Gamma^{p}\left(S_{\lambda}\right)$ does not have any $s l_{2}$-invariants and, hence, $\operatorname{Ker}\left\{\widetilde{\Theta}_{\underline{s}_{2}}, \Gamma^{p}\left(S_{\lambda}\right) \otimes \mathcal{O}_{X}\right\}$ does not have any global sections. We conclude that the kernel bundles associated to the dual modules $S^{p}\left(S_{\lambda}\right)$ and $\Gamma^{p}\left(S_{\lambda}\right)$ are nonisomorphic.

We continue our consideration of $\mathrm{SL}_{2(1)} \equiv \underline{s}_{2}$ in the following proposition.

Proposition 6.3. Let $G=\underline{s}_{2}$, let $S_{\lambda}$ be the irreducible $k G$-module of highest weight $\lambda, 0 \leq \lambda \leq p-1$, and let $P_{\lambda} \rightarrow S_{\lambda}$ be the projective cover of $S_{\lambda}$. Then:

$i^{*}\left(\operatorname{Ker}\left\{\widetilde{\Theta}_{G}, P_{\lambda} \otimes \mathcal{O}_{\mathbb{P}(\mathrm{G})}\right\}\right) \simeq\left\{\begin{array}{l}\mathcal{O}_{\mathbb{P}^{1}}(1-p) \quad \text { if } \lambda=p-1, \\ \mathcal{O}_{\mathbb{P}^{1}}(\lambda-2(p-1)) \oplus \mathcal{O}_{\mathbb{P}^{1}}(-\lambda) \quad \text { if } 0 \leq \lambda \leq p-2,\end{array}\right.$

where $i: \mathbb{P}^{1} \rightarrow \mathbb{P}(\mathrm{G})$ is the isomorphism (5.8.1).

Proof. For $\lambda=p-1, P_{\lambda}=$ St is the Steinberg module for $s l_{2}$ and is irreducible. Hence, in this case the statement follows from Proposition 5.9.

The decomposition series of $P_{\lambda}$ for $0 \leq \lambda<p-1$ is represented by the following diagram (see [14, 2.4]):

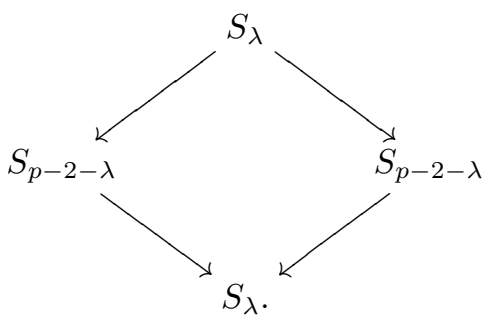

Thus, we have a short exact sequence of $\mathrm{SL}_{2}$-modules:

$$
0 \rightarrow V_{2 p-2-\lambda} \rightarrow P_{\lambda} \rightarrow S_{\lambda} \rightarrow 0 .
$$

By Proposition 5.9] it suffices to prove that $V_{2 p-2-\lambda} \subset P_{\lambda}$ induces an isomorphism

$$
\operatorname{Ker}\left\{\widetilde{\Theta}_{G}, V_{2 p-2-\lambda} \otimes \mathcal{O}_{\mathbb{P}(\mathrm{G})}\right\} \simeq \operatorname{Ker}\left\{\widetilde{\Theta}_{G}, P_{\lambda} \otimes \mathcal{O}_{\mathbb{P}(\mathrm{G})}\right\} .
$$

By Theorem 4.13(8) and Nakayama's Lemma, it suffices to prove that

$$
\operatorname{Ker}\left\{\theta_{x}, V_{2 p-2-\lambda} \otimes k(x)\right\} \rightarrow \operatorname{Ker}\left\{\theta_{x}, P_{\lambda} \otimes k(x)\right\}
$$

is an isomorphism for all $x \in \mathbb{P}(\mathrm{G})$. This last statement follows from the observation that the Jordan decomposition of $\theta_{x}$ on both $V_{\lambda}$ and $P_{\lambda}$ consists of two blocks: on $P_{\lambda}$, because $P_{\lambda}$ is projective of dimension $2 p$; and on $V_{2 p-2-\lambda}$, as discussed in Proposition 5.9 .

If $X$ is an algebraic variety over $k$ (for example, $X=\mathbb{P}(G)$ ), then $K_{0}(X)$ denotes the Grothendieck group of algebraic vector bundles over $X$ (i.e., finitely generated, locally free $\mathcal{O}_{X}$-modules) defined as the free abelian group on the set of isomorphism classes of such vector bundles modulo relations given by short exact sequences. We shall also consider $K_{0}^{\oplus}(X)$ defined as the free abelian group on the same set of generators modulo relations given by split short exact sequences. Thus, there is a canonical surjective homomorphism

$$
K_{0}^{\oplus}(X) \rightarrow K_{0}(X) .
$$


Definition 6.4. Let $G$ be an infinitesimal group scheme and let $K_{0}(k G)$ denote the Grothendieck group of finitely generated projective $k G$-modules. For any $j, 0 \leq$ $j \leq p-1$, the homomorphism

$$
\kappa_{G, j}^{\oplus}: K_{0}(k G) \rightarrow K_{0}^{\oplus}(\mathbb{P}(G))
$$

is defined by sending a projective $k G$-module $Q$ to $\operatorname{Ker}\left\{\widetilde{\Theta}_{G}^{j}, Q \otimes \mathcal{O}_{\mathbb{P}(G)}\right\}$. We define $\underline{\kappa}_{G}^{\oplus}$ by

$$
\underline{\kappa}_{G}^{\oplus}=\left(\kappa_{G, 1}^{\oplus}, \ldots \kappa_{G, p}^{\oplus}\right): K_{0}(k G) \rightarrow K_{0}^{\oplus}(\mathbb{P}(G))^{\oplus p} .
$$

Moreover, the homomorphism

$$
\kappa_{G, j}: K_{0}(k G) \rightarrow K_{0}(\mathbb{P}(G))
$$

is defined to be the composition of $\tilde{\kappa}_{G, j}^{\oplus}$ with the canonical projection $K_{0}^{\oplus}(\mathbb{P}(G)) \rightarrow$ $K_{0}(\mathbb{P}(G))$, and the homomorphism

$$
\underline{\kappa}_{G}: K_{0}(k G) \rightarrow K_{0}(\mathbb{P}(G))^{\oplus p}
$$

is defined to be the composition of $\underline{\kappa}_{G}$ with the canonical projection $K_{0}^{\oplus}(\mathbb{P}(G))^{\oplus p} \rightarrow$ $K_{0}(\mathbb{P}(G))^{\oplus p}$.

We shall omit the subscript $G$ in $\kappa_{G}$ when the group scheme is clear from the context. Note that since $\widetilde{\Theta}^{p}=0, \kappa_{p}=\left[Q \otimes \mathcal{O}_{\mathbb{P}(\mathrm{G})}\right]$.

The dimension of the global sections function,

$$
\mathcal{E} \mapsto \operatorname{dim} \Gamma(\mathbb{P}(\mathrm{G}), \mathcal{E}),
$$

extends to a homomorphism

$$
\rho: K_{0}^{\oplus}(\mathbb{P}(G)) \rightarrow \mathbb{Z} .
$$

Observe that since $\Gamma(\mathbb{P}(G),-)$ is not right exact for non-split exact sequences of algebraic vector bundles on $\mathbb{P}(G)$, $\rho$ does not factor through $K_{0}(\mathbb{P}(G)$ ).

Proposition 6.5. Let $G=\underline{s}_{2}$. The composition

$$
\rho \circ \underline{\kappa}^{\oplus}: K_{0}(k G) \rightarrow K_{0}^{\oplus}(\mathbb{P}(\mathrm{G}))^{\oplus p} \rightarrow \mathbb{Z}^{\oplus p}
$$

is a rational isomorphism.

Proof. Recall that $K_{0}(k G)=K_{0}\left(u\left(s l_{2}\right)\right) \simeq \mathbb{Z}^{\oplus p}$, spanned by the projective indecomposable $u\left(s l_{2}\right)$-modules $P_{\lambda}, 0 \leq \lambda \leq p-1$. By Proposition 5.21] the global sections of $\left.\operatorname{Ker}\left\{\widetilde{\Theta}_{G}^{j}, P_{\lambda} \otimes \mathcal{O}_{\mathbb{P}(\mathrm{G})}\right\}\right)$ are rational $\mathrm{SL}_{2}$-submodules of $P_{\lambda}$. Hence, $\Gamma\left(\mathbb{P}(\mathrm{G}), \operatorname{Ker}\left\{\widetilde{\Theta}_{G}^{j}, P_{\lambda} \otimes \mathcal{O}_{\mathbb{P}(\mathrm{G})}\right\}\right) \neq 0$ if and only if $S_{\lambda}$, which is the socle of $P_{\lambda}$, belongs to the global sections. Hence,

$\Gamma\left(\mathbb{P}(\mathrm{G}), \operatorname{Ker}\left\{\widetilde{\Theta}_{G}^{j}, P_{\lambda} \otimes \mathcal{O}_{\mathbb{P}(\mathrm{G})}\right\}\right) \neq 0 \quad \Leftrightarrow \quad \widetilde{\Theta}^{j}\left(S_{\lambda} \otimes \mathcal{O}_{\mathbb{P}(\mathrm{G})}\right)=0 \quad \Leftrightarrow \quad \theta_{v}^{j}\left(S_{\lambda}\right)=0$ for any $v \in V(G) \simeq \mathcal{N}\left(s l_{2}\right)$. Since $S_{\lambda}$ is a module of constant Jordan type $[\lambda+1]$, $\theta_{v}^{j}$ annihilates $S_{\lambda}$ if and only if $j>\lambda$. Thus,

$$
\Gamma\left(\mathbb{P}(\mathrm{G}), \operatorname{Ker}\left\{\widetilde{\Theta}_{G}^{j}, P_{\lambda} \otimes \mathcal{O}_{\mathbb{P}(\mathrm{G})}\right\}\right) \neq 0 \text { if and only if } j>\lambda \text {. }
$$

Moreover, the decomposition series for $P_{\lambda}$ (6.3.1) implies that the Jordan type of any rational submodule of $P_{\lambda}$ that is larger than the socle $S_{\lambda}$ has a Jordan block of size $p$. Hence,

$$
\Gamma\left(\mathbb{P}(\mathrm{G}), \operatorname{Ker}\left\{\widetilde{\Theta}_{G}^{\lambda+1}, P_{\lambda} \otimes \mathcal{O}_{\mathbb{P}(\mathrm{G})}\right\}\right)=S_{\lambda} .
$$


Note that the last equality holds trivially for $\lambda=p-1$, since in this case the kernel bundle is the entire free sheaf $P_{p-1} \otimes \mathcal{O}_{\mathbb{P}(\mathrm{G})}$, where $P_{p-1}=S_{p-1}$ is the Steinberg module for $s l_{2}$.

We conclude that the homomorphism

$$
\rho \circ \underline{\kappa}^{\oplus}: K_{0}(k G) \simeq \mathbb{Z}^{\oplus p} \rightarrow \mathbb{Z}^{\oplus p}
$$

is given by a non-singular upper-triangular matrix

$$
\left[\begin{array}{ccccc}
1 & \cdots & & & \cdot \\
0 & 2 & \ldots & & \cdot \\
0 & 0 & 3 & \cdots & . \\
\vdots & \vdots & \vdots & \ddots & \vdots \\
0 & 0 & 0 & 0 & p
\end{array}\right] .
$$

Hence, $\rho \circ \underline{\kappa}^{\oplus}$ is a rational isomorphism.

In contrast with Proposition [6.5, we have the following computations for $\kappa_{1}$ : $K_{0}(k G) \rightarrow K_{0}(\mathbb{P}(\mathrm{G}))$ and $\underline{\kappa}: K_{0}(k G) \rightarrow K_{0}(\mathbb{P}(\mathrm{G}))^{\oplus p}$ for $G=\underline{s} l_{2}$.

Lemma 6.6. Let $G=s l_{2}$, and denote by St the Steinberg module for $s l_{2}$. The image of the homomorphism

$$
\kappa_{1}: K_{0}(k G) \rightarrow K_{0}(\mathbb{P}(\mathrm{G}))
$$

is generated by $\kappa_{1}(\mathrm{St})$. Consequently, $\mathrm{rk} \kappa_{1}=1$.

Proof. The isomorphism $i: \mathbb{P}(\mathrm{G}) \simeq \mathbb{P}^{1}$ (5.8.1) induces an isomorphism $K_{0}(\mathbb{P}(\mathrm{G})) \simeq$ $K_{0}\left(\mathbb{P}^{1}\right)$. We denote by $\kappa_{1}$ the composition $K_{0}(k G) \rightarrow K_{0}(\mathbb{P}(\mathrm{G})) \stackrel{\sim}{\rightarrow} K_{0}\left(\mathbb{P}^{1}\right)$. Clearly, it suffices to prove the statement of the lemma for this composition.

Let $a_{n}=\left[\mathcal{O}_{\mathbb{P}^{1}}(-n)\right] \in K_{0}\left(\mathbb{P}^{1}\right), n \in \mathbb{Z}$. Then $a_{0}=\left[\mathcal{O}_{\mathbb{P}^{1}}\right], a_{1}=\left[\mathcal{O}_{\mathbb{P}^{1}}(-1)\right]$ generate $K_{0}\left(\mathbb{P}^{1}\right) \simeq \mathbb{Z}^{\oplus 2}$. Using the short exact sequence of $\mathcal{O}_{\mathbb{P}^{1} \text {-modules }}$

$$
0 \rightarrow \mathcal{O}_{\mathbb{P}^{1}}(-(n+1)) \rightarrow \mathcal{O}_{\mathbb{P}^{1}}(-n) \oplus \mathcal{O}_{\mathbb{P}^{1}}(-n) \rightarrow \mathcal{O}_{\mathbb{P}^{1}}(-(n-1)) \rightarrow 0,
$$

we obtain the recurrence relation $a_{n+1}=2 a_{n}-a_{n-1}$. By induction, $a_{n}=n a_{1}-$ $(n-1) a_{0}$.

By Proposition 6.3, for $0 \leq \lambda<p-1$,

$$
\kappa_{1}\left(P_{\lambda}\right)=\left[\mathcal{O}_{\mathbb{P}^{1}}(-\lambda)\right]+\left[\mathcal{O}_{\mathbb{P}^{1}}(\lambda-2(p-1)] .\right.
$$

Hence, $\kappa_{1}\left(P_{\lambda}\right)=a_{\lambda}+a_{2(p-1)-\lambda}=\lambda a_{1}-(\lambda-1) a_{0}+(2(p-1)-\lambda) a_{1}-(2(p-1)-$ $\lambda-1) a_{0}=2(p-1) a_{1}-2(p-2) a_{0}$. Moreover,

$$
\kappa_{1}(\mathrm{St})=\left[\mathcal{O}_{\mathbb{P}^{1}}(1-p)\right]=(p-1) a_{1}-(p-2) a_{0}
$$

by Proposition 5.9. Hence,

$$
\kappa_{1}\left(P_{\lambda}\right)=2 \kappa_{1}(\mathrm{St})
$$

for $0 \leq \lambda \leq p-2$, which proves the statement.

The second conclusion of the following proposition is in sharp contrast with Proposition 6.5.

Proposition 6.7. Let $G=\underline{s l}_{2}$, and denote by St the Steinberg module for $s l_{2}$. Then:

(1) The image of $\kappa_{j}: K_{0}(k G) \rightarrow K_{0}(\mathbb{P}(\mathrm{G}))$ is generated by $\kappa_{j}(\mathrm{St})$. 
(2) The image of

$$
\underline{\kappa}: K_{0}(k G) \rightarrow K_{0}(\mathbb{P}(\mathrm{G}))^{\oplus p}
$$

is generated by $\underline{\kappa}(\mathrm{St})$ and, consequently, has rank 1 .

Proof. As in the proof of Lemma 6.6, we identify $K_{0}(\mathbb{P}(\mathrm{G}))$ with $K_{0}\left(\mathbb{P}^{1}\right)$. We have a short exact sequence of bundles:

$$
\begin{aligned}
& 0 \longrightarrow \operatorname{Ker}\left\{\widetilde{\Theta}, P_{\lambda} \otimes \mathcal{O}_{\mathbb{P}(\mathrm{G})}\right\} \longrightarrow \operatorname{Ker}\left\{\widetilde{\Theta}^{j}, P_{\lambda} \otimes \mathcal{O}_{\mathbb{P}(\mathrm{G})}\right\} \\
& \stackrel{\widetilde{\Theta}}{\longrightarrow} \operatorname{Ker}\left\{\widetilde{\Theta}^{j-1}, P_{\lambda} \otimes \mathcal{O}_{\mathbb{P}(\mathrm{G})}\right\}(2) \longrightarrow 0 .
\end{aligned}
$$

Indeed, the composition is clearly zero, and the first map is an embedding. Moreover, by Theorem 4.13. the specialization of this sequence at any point $x \in \mathbb{P}(\mathrm{G})$ looks as follows:

$0 \longrightarrow \operatorname{Ker}\left\{\theta_{x}, P_{\lambda} \otimes k(x)\right\} \longrightarrow \operatorname{Ker}\left\{\theta_{x}^{j}, P_{\lambda} \otimes k(x)\right\} \stackrel{\theta_{x}}{\longrightarrow} \operatorname{Ker}\left\{\theta_{x}^{j-1}, P_{\lambda} \otimes k(x)\right\} \longrightarrow 0$.

The projectivity of $P_{\lambda}$ implies that this sequence is exact. Hence, 6.7.1) is exact by Nakayama's Lemma.

We conclude that in $K_{0}\left(\mathbb{P}^{1}\right)$,

$$
\kappa_{j}\left(P_{\lambda}\right)=\kappa_{1}\left(P_{\lambda}\right)+\kappa_{j-1}\left(P_{\lambda}\right)(2) .
$$

By Lemma 6.6, $\kappa_{1}\left(P_{\lambda}\right)=2 \kappa_{1}(\mathrm{St})$ for $0 \leq \lambda \leq p-2$. Applying induction, we get $\kappa_{j}\left(P_{\lambda}\right)=2 \kappa_{1}(\mathrm{St})+2 \kappa_{j-1}(\mathrm{St})(2)$. Now applying formula (6.7.2) to the Steinberg module, we get $2 \kappa_{1}(\mathrm{St})+2 \kappa_{j-1}(\mathrm{St})(2)=2 \kappa_{j}(\mathrm{St})$. To summarize,

$$
\kappa_{j}\left(P_{\lambda}\right)=\left\{\begin{array}{l}
\kappa_{j}(\mathrm{St}), \quad \lambda=p-1, \\
2 \kappa_{j}(\mathrm{St}), \quad 0 \leq \lambda \leq p-2,
\end{array}\right.
$$

which implies (1). Moreover, we conclude that $\underline{\kappa}: K_{0}(k G) \rightarrow K_{0}\left(\mathbb{P}^{1}\right)^{\oplus p}$ is given by the formula

$$
\underline{\kappa}\left(P_{\lambda}\right)=\left\{\begin{array}{l}
\left(\kappa_{1}(\mathrm{St}), \kappa_{2}(\mathrm{St}), \ldots, \kappa_{p}(\mathrm{St})\right), \quad \lambda=p-1, \\
\left(2 \kappa_{1}(\mathrm{St}), 2 \kappa_{1}(\mathrm{St}), \ldots, 2 \kappa_{p}(\mathrm{St})\right)=2 \underline{\kappa}(\mathrm{St}), \quad 0 \leq \lambda \leq p-2,
\end{array}\right.
$$

which proves (2).

Proposition 6.9 gives us some information about the behavior of $\kappa$ and $\kappa^{\oplus}$ with respect to products. First, we need a trivial linear algebra lemma.

Lemma 6.8. Let $V, W$ be vector spaces over a field $k$. Let $\left\{v_{1}, \ldots, v_{r}\right\}$ be a basis of $V$ and $\left\{w_{1}, \ldots, w_{s}\right\}$ be a basis of $W$. Let $v \in V, w \in W$, and let $X \subset V \oplus W$ be the span of $\left\{\left(v_{1}, w\right), \ldots,\left(v_{r}, w\right),\left(v, w_{1}\right), \ldots,\left(v, w_{s}\right)\right\}$. Then the dimension of $X$ is at least $r+s-1$.

Proof. If $v=0$ and $w=0$, then we obviously have $\operatorname{dim} X=r+s$. We assume $v \neq 0$. Observe that

$$
X \longrightarrow(V \oplus W) /\langle(v, 0)+(0, w)\rangle
$$

is surjective (thus has image of dimension $r+s-2$ ). Let $v=a_{1} v_{1}+\cdots+a_{r} v_{r}$. Then the vector $a_{1}\left(v_{1}, w\right)+\cdots+a_{r}\left(v_{r}, w\right)=(v, w)$ is non-trivial since $v \neq 0$ and belongs to the kernel of the projection (6.8.1). Hence, $\operatorname{dim} X \geq r+s-1$. 
Proposition 6.9. Let $G_{1}$ and $G_{2}$ be infinitesimal group schemes, and let $G=$ $G_{1} \times G_{2}$. Then

$$
\operatorname{rk} \kappa_{G}^{\oplus} \geq \operatorname{rk} \kappa_{G_{1}}^{\oplus}+\operatorname{rk} \kappa_{G_{2}}^{\oplus}-1,
$$

where $\kappa^{\oplus}=\kappa_{j}^{\oplus}$ for any $j, 1 \leq j \leq p-1$.

Proof. Let $i_{\ell}^{*}: K_{0}^{\oplus}(\mathbb{P}(\mathrm{G})) \rightarrow K_{0}^{\oplus}\left(\mathbb{P}\left(G_{\ell}\right)\right)$ be the map induced by the pull-back of vector bundles along the embedding $\mathbb{P}\left(G_{\ell}\right) \hookrightarrow \mathbb{P}(\mathrm{G})$, for $\ell=1,2$. We consider the composition

$$
K_{0}(k G) \stackrel{\kappa^{\oplus}}{\longrightarrow} K_{0}^{\oplus}(\mathbb{P}(\mathrm{G})) \stackrel{i_{1}^{*}+i_{2}^{*}}{\longrightarrow} K_{0}^{\oplus}\left(\mathbb{P}\left(G_{1}\right)\right) \oplus K_{0}^{\oplus}\left(\mathbb{P}\left(G_{2}\right)\right) .
$$

Let $P$ be a projective $k G_{1}$-module of dimension $m$ and $Q$ be a projective $k G_{2^{-}}$ module of dimension $n$. Then $P \otimes Q$ is a projective $k G$-module. By Corollary [5.4,

$$
\begin{array}{r}
\left(i_{1}^{*}+i_{2}^{*}\right) \circ \kappa_{G}^{\oplus}(P \otimes Q)=\left(i_{1}^{*} \circ \kappa_{G}^{\oplus}(P \otimes Q), i_{2}^{*} \circ \kappa_{G}^{\oplus}(P \otimes Q)\right) \\
=\left(n \kappa_{G_{1}}^{\oplus}(P), m \kappa_{G_{2}}^{\oplus}(Q)\right) .
\end{array}
$$

Let $\left\{P_{1}, \ldots, P_{r}\right\}$ be projective $k G_{1}$-modules of dimensions $\left\{p_{1}, \ldots, p_{r}\right\}$ such that

$$
\left\{\kappa_{G_{1}}^{\oplus}\left(P_{1}\right), \ldots, \kappa_{G_{1}}^{\oplus}\left(P_{r}\right)\right\}
$$

are linearly independent generators of $\operatorname{Im} \kappa_{G_{1}}^{\oplus} \subset K_{0}^{\oplus}\left(\mathbb{P}\left(G_{1}\right)\right)$, so that $\operatorname{rk} \kappa_{G_{1}}^{\oplus}=r$. Similarly, let $\left\{Q_{1}, \ldots, Q_{s}\right\}$ be projective $k G_{2}$-modules such that

$$
\left\{\kappa_{G_{2}}^{\oplus}\left(Q_{1}\right), \ldots, \kappa_{G_{2}}^{\oplus}\left(Q_{s}\right)\right\}
$$

are linearly independent generators of $\operatorname{Im} \kappa_{G_{2}}^{\oplus} \subset K_{0}^{\oplus}\left(\mathbb{P}\left(G_{2}\right)\right)$, so that $\operatorname{rk} \kappa_{G_{2}}^{\oplus}=s$. Finally, let $P$ be any projective $k G_{1}$-module, $m=\operatorname{dim}_{k} P$, and $Q$ be any projective $k G_{2}$-module, $n=\operatorname{dim}_{k} Q$. Consider

$$
S=\operatorname{Span}\left\{P_{1} \otimes Q, P_{2} \otimes Q, \ldots, P_{r} \otimes Q, P \otimes Q_{1}, P \otimes Q_{2}, \ldots, P \otimes Q_{s}\right\} \subset K_{0}(k G) .
$$

Then the image of $\left(i_{1}^{*}+i_{2}^{*}\right) \circ \kappa_{G}$ contains

$$
\left\{\left(n \kappa_{G_{1}}^{\oplus}\left(P_{1}\right), p_{1} \kappa_{G_{2}}^{\oplus}(Q)\right),\left(n \kappa_{G_{1}}^{\oplus}\left(P_{2}\right), p_{2} \kappa_{G_{2}}^{\oplus}(Q)\right), \ldots,\left(n \kappa_{G_{1}}^{\oplus}\left(P_{r}\right), p_{r} \kappa_{G_{2}}^{\oplus}(Q)\right)\right\}
$$

and

$$
\left\{\left(q_{1} \kappa_{G_{1}}^{\oplus}(P), m \kappa_{G_{2}}^{\oplus}\left(Q_{1}\right)\right),\left(q_{2} \kappa_{G_{1}}^{\oplus}(P), m \kappa_{G_{2}}^{\oplus}\left(Q_{2}\right)\right), \ldots,\left(q_{s} \kappa_{G_{1}}^{\oplus}(P), m \kappa_{G_{2}}^{\oplus}\left(Q_{s}\right)\right)\right\} .
$$

Since all the coefficients $m, n, p_{i}, q_{j}$ are positive, Lemma 6.8 implies that the dimension of the image of $\left(i_{1}^{*}+i_{2}^{*}\right) \circ \kappa_{G}^{\oplus}$ is at least $r+s-1$.

Remark 6.10. As the reader can easily check, Proposition 6.9 and its proof hold for $\kappa: K_{0}(k G) \rightarrow K_{0}(\mathbb{P}(G))$ in place of $\kappa^{\oplus}: K_{0}(k G) \rightarrow K_{0}^{\oplus}(\mathbb{P}(G))$.

Observe that $\mathbb{P}\left(\underline{s l_{2}^{\oplus r}}\right) \simeq \mathbb{P}^{2 r-1}$, the join of $r$ copies of $\mathbb{P}\left(\underline{s} l_{2}\right) \simeq \mathbb{P}^{1}$.

Corollary 6.11. Let $G=\underline{s l}_{2}^{\times r}$ be the infinitesimal group scheme corresponding to the restricted Lie algebra $s l_{2}^{\oplus r}$. Then

$$
\kappa_{1}^{\oplus}: K_{0}(k G) \rightarrow K_{0}^{\oplus}\left(\mathbb{P}^{2 r-1}\right)
$$

has rank at least $r(p-1)+1$.

Proof. For $r=1, \kappa_{\underline{s l}_{2}}^{\oplus}: K_{0}\left(u\left(s l_{2}\right)\right) \rightarrow K_{0}^{\oplus}\left(\mathbb{P}^{1}\right)$ is injective by Proposition 6.3 and, hence, has rank $p$. The statement now follows by induction and Proposition 6.9. 
Recall that if $H \hookrightarrow G$ is a subgroup scheme of a finite group scheme $G$, then $k G$ is free as a $k H-$ module $[24,2.4]$. Hence, the restriction functor res : $(k G-\bmod ) \rightarrow$ $(k H-\bmod )$ induces a well-defined map on $K$-groups: res* $^{*} K_{0}(k G) \rightarrow K_{0}(k H)$. The commutativity of the diagram (5.3.1) implies that restriction commutes with $\kappa_{j}$. That is, we have a commutative diagram

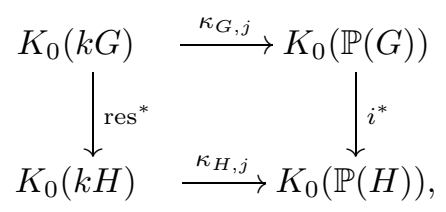

where $i: \mathbb{P}(H) \hookrightarrow \mathbb{P}(\mathrm{G})$ is the closed embedding of projective varieties induced by the embedding of group schemes and $i^{*}: K_{0}(\mathbb{P}(G)) \rightarrow K_{0}(\mathbb{P}(H))$ is the pull-back via $i$ of locally free $\mathcal{O}_{P(G)}$-modules. An analogous commutative diagram holds for $\kappa^{\oplus}$.

Proposition 6.12. Let $G$ be an infinitesimal group scheme, and let $H \simeq \underline{s}_{2}^{\times r} \subset G$ be a closed subgroup scheme with the property that res* $: K_{0}(k G) \rightarrow K_{0}(k H)$ is rationally surjective. Then the composition

$$
K_{0}(k G) \stackrel{\kappa_{1}^{\oplus}}{\longrightarrow} K_{0}^{\oplus}(\mathbb{P}(\mathrm{G})) \stackrel{i^{*}}{\longrightarrow} K_{0}^{\oplus}(\mathbb{P}(H)) \simeq K_{0}^{\oplus}\left(\mathbb{P}^{2 r-1}\right)
$$

has rank at least $r(p-1)+1$.

Proof. We apply the diagram (6.11.1) for $\kappa_{1}^{\oplus}$ :

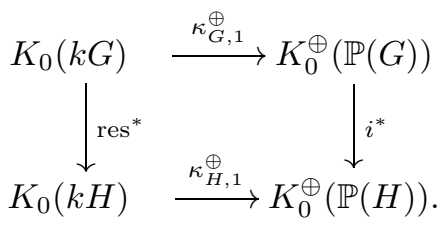

Since res* is assumed to be rationally surjective, $\operatorname{rk}\left(i^{*} \circ \kappa_{G, 1}^{\oplus}\right)=\operatorname{rk} \kappa_{H, 1}^{\oplus}$. Since $H=\underline{s l}_{2}^{\times r}, \mathrm{rk} \kappa_{H, 1}^{\oplus} \geq r(p-1)+1$ by Corollary 6.11. This proves the statement.

For the rest of this computational section, we calculate some examples of bundles for $E=\mathbb{G}_{a(1)} \times \mathbb{G}_{a(1)}$. We have $k E \simeq k[x, y] /\left(x^{p}, y^{p}\right)$. Let $X_{n}$ be a $(2 n+1)$ dimensional "zig-zag" module. Pictorially, we represent $X_{n}$ by the following diagram:

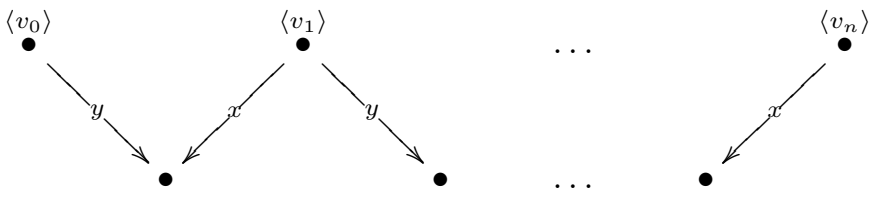

It is straightforward to check that $X_{n}$ has constant Jordan type $n[2]+[1]$ (see [8, $\S 2])$. We proceed to prove that for any integer $m$ we can obtain the line bundle $\mathcal{O}_{\mathbb{P}^{1}}(m)$ on $\mathbb{P}(E)=\mathbb{P}^{1}$ by applying our constructions to some $X_{n}$ or its linear dual $X_{n}^{\#}$.

Note that for $X_{n}$, the map

$$
\widetilde{\Theta}_{E}: X_{n} \otimes \mathcal{O}_{\mathbb{P}^{1}} \longrightarrow X_{n} \otimes \mathcal{O}_{\mathbb{P}^{1}}(1)
$$


as defined in (4.6.1) has nilpotentcy degree 2. Hence, there is an inclusion

$$
\operatorname{Im}\left\{\widetilde{\Theta}_{E}, X_{n} \otimes \mathcal{O}_{\mathbb{P}^{1}}\right\} \subset \operatorname{Ker}\left\{\widetilde{\Theta}_{E}, X_{n} \otimes \mathcal{O}_{\mathbb{P}^{1}}(1)\right\} .
$$

We, therefore, may define a subquotient sheaf of the free sheaf $X_{n} \otimes \mathcal{O}_{\mathbb{P}^{1}}$ as

$$
\mathcal{X}_{n}:=\operatorname{Ker}\left\{\widetilde{\Theta}_{E}, X_{n} \otimes \mathcal{O}_{\mathbb{P}^{1}}\right\} / \operatorname{Im}\left\{\widetilde{\Theta}_{E}(-1), X_{n} \otimes \mathcal{O}_{\mathbb{P}^{1}}(-1)\right\} .
$$

Arguing as in the proof of Proposition 5.16, one verifies that $\mathcal{X}_{n}$ is locally free with the fiber at a point $t \in \mathbb{P}^{1}$ isomorphic to the 1-dimensional vector space $\frac{\operatorname{Ker}\left\{\theta_{t}: X_{n, k(t)} \rightarrow X_{n, k(t)}\right\}}{\operatorname{Im}\left\{\theta_{t}: X_{n, k(t)} \rightarrow X_{n, k(t)}\right\}}$. Hence, $\mathcal{X}_{n}$ is a line bundle. The linear dual $X_{n}^{\#}$ of $X_{n}$ is represented by the diagram

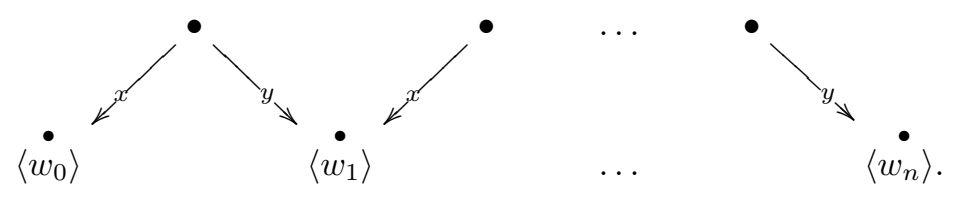

Define the subquotient sheaf of $X_{n}^{\#} \otimes \mathcal{O}_{\mathbb{P}^{1}}$ as

$$
\mathcal{Y}_{n}:=\operatorname{Ker}\left\{\widetilde{\Theta}_{E}, X_{n}^{\#} \otimes \mathcal{O}_{\mathbb{P}^{1}}\right\} / \operatorname{Im}\left\{\widetilde{\Theta}_{E}(-1), X_{n}^{\#} \otimes \mathcal{O}_{\mathbb{P}^{1}}(-1)\right\}
$$

Proposition 6.13. $\mathcal{X}_{n} \simeq \mathcal{O}_{\mathbb{P}^{1}}(-n), \mathcal{Y}_{n} \simeq \mathcal{O}_{\mathbb{P}^{1}}(n)$

Proof. Let $k[s, t]=k\left[\mathbb{A}^{2}\right] \simeq k[V(E)]$. The universal $p$-nilpotent operator $\Theta_{E} \in$ $k[x, y] /\left(x^{p}, y^{p}\right) \otimes k[s, t]$ is given by

$$
\Theta_{E}=x s+y t
$$

(see, for example, Example [2.7). We shall identify the graded $k[s, t]$-module $\operatorname{Ker}\left\{\Theta_{E}, X_{n} \otimes k[s, t]\right\} / \operatorname{Im}\left\{\Theta_{E}, X_{n} \otimes k[s, t]\right\}$, thereby determining the vector bundle $\mathcal{X}_{n}$. It is easy to see that $\operatorname{Im}\left\{\Theta_{E}, X_{n} \otimes k[s, t]\right\}$ is generated by the bottom row of the diagram representing $X_{n}$ as a $k[s, t]$-module and that $\operatorname{Ker}\left\{\Theta_{E}, X_{n} \otimes k[s, t]\right\}$ is generated by the same bottom row and the vector $s^{n} v_{0}+s^{n-1} t v_{1}+\cdots+t^{n} v_{n}$. Hence, $\operatorname{Ker}\left\{\Theta_{E}, X_{n} \otimes k[s, t]\right\} / \operatorname{Im}\left\{\Theta_{E}, X_{n} \otimes k[s, t]\right\}$ is generated by $s^{n} v_{0}+s^{n-1} t v_{1}+\cdots+t^{n} v_{n}$ as a $k[s, t]$-module. Since the generator is in degree $n$, we conclude that the corresponding locally free sheaf of rank 1 is $\mathcal{O}_{\mathbb{P}^{1}}(-n)$.

We now compute $\mathcal{Y}_{n}$. The graded $k[s, t]$-module $\operatorname{Ker}\left\{\Theta_{E}, X_{n}^{\#} \otimes k[s, t]\right\}$ is generated by $\left\langle w_{0}, \ldots, w_{n}\right\rangle$ in degree 0 , and $\operatorname{Im}\left\{\Theta_{E}, X_{n}^{\#} \otimes k[s, t](-1)\right\}$ is generated by $\left\langle s w_{0}+t w_{1}, s w_{1}+t w_{2}, \ldots, s w_{n-1}+t w_{n}\right\rangle$, also in degree 0 . Hence, on $U_{0}=\mathbb{P}^{1}-Z(s)$, the restriction of $\mathcal{Y}_{n}$ is generated by $w_{n}$, with $w_{0}=\left(-\frac{t}{s}\right)^{n} w_{n}$. We map $\mathcal{Y}_{n}\left(U_{0}\right)$ to $K=k(t / s)$, the residue field at the generic point of $\mathbb{P}^{1}$, by sending $w_{n}$ to 1 . The image of $w_{0}$ is $\left(-\frac{t}{s}\right)^{n}$. On the other affine piece, $U_{1}=\mathbb{P}^{1}-Z(t)$, the restriction of $\mathcal{Y}_{n}$ is generated by $w_{0}$, with the relation $w_{n}=\left(-\frac{s}{t}\right)^{n} w_{0}$. We map this to $K=k(s / t)$ by sending $w_{0}$ to $\left(-\frac{t}{s}\right)^{n}$. Hence, the vector bundle is given by the Cartier divisor $\left(U_{0}, 1\right),\left(U_{1},\left(-\frac{t}{s}\right)^{n}\right)$. This divisor is equivalent to the Cartier divisor $\left(U_{0}, 1\right),\left(U_{1},\left(\frac{t}{s}\right)^{n}\right)$ which corresponds to the line bundle $\mathcal{O}_{\mathbb{P}^{1}}(n)$. Hence, $\mathcal{Y}_{n} \simeq \mathcal{O}_{\mathbb{P}^{1}}(n)$.

In the next proposition we calculate explicitly the line bundles corresponding to the syzygies of the trivial modules, $\Omega^{n} k$. For convenience, we use the notation $\mathcal{H}^{[1]}(M)$ for the bundle $\mathcal{M}^{[1]}$ associated to $M$ as defined in (5.14). 
Proposition 6.14. Let $E=\mathbb{G}_{a(1)}^{\times r}$. Then

$$
\mathcal{H}^{[1]}\left(\Omega^{n} k\right) \simeq \begin{cases}\mathcal{O}_{\mathbb{P}^{r-1}}\left(-\frac{n p}{2}\right) & \text { if } n \text { is even }, \\ \mathcal{O}_{\mathbb{P}^{r-1}}\left(-\frac{n+1}{2} p+1\right) & \text { if } n \text { is odd }\end{cases}
$$

for $p$ odd and

$$
\mathcal{H}^{[1]}\left(\Omega^{n} k\right) \simeq \mathcal{O}_{\mathbb{P}^{r-1}}(-n)
$$

for $p=2$.

Proof. Let $r=2$, and assume $n \geq 0$. As in the proof of Proposition 6.13, the universal operator $\Theta_{E}$ equals $s x+t y$, where $k[V(E)]=k[s, t]$. The structure of a minimal $k E \simeq k[x, y] /\left(x^{p}, y^{p}\right)$-projective resolution $P_{\bullet} \rightarrow k$ is well known [10, with $P_{n-1}=k E^{\times n}$. A set of generators $a_{1}, \ldots, a_{n}$ for $P_{n-1}$ can be chosen so that $\Omega^{n}(k)$ is the submodule generated by the elements

$$
x^{p-1} a_{1}, \quad y a_{1}-x a_{2}, \quad y^{p-1} a_{2}-x^{p-1} a_{3}, \quad y a_{3}-x a_{4}, \quad \ldots, y a_{n-1}-x a_{n}, \quad y^{p-1} a_{n}
$$

for $n$ even, and

$$
x a_{1}, \quad y a_{1}-x^{p-1} a_{2}, \quad y^{p-1} a_{2}-x a_{3}, \quad y a_{3}-x^{p-1} a_{4}, \quad \ldots, \quad y^{p-1} a_{n-1}-x a_{n}, \quad y a_{n}
$$

for $n$ odd.

Let $n$ be even. For illustrational purposes, we include a picture of $\Omega^{4} k$ for $p=3$

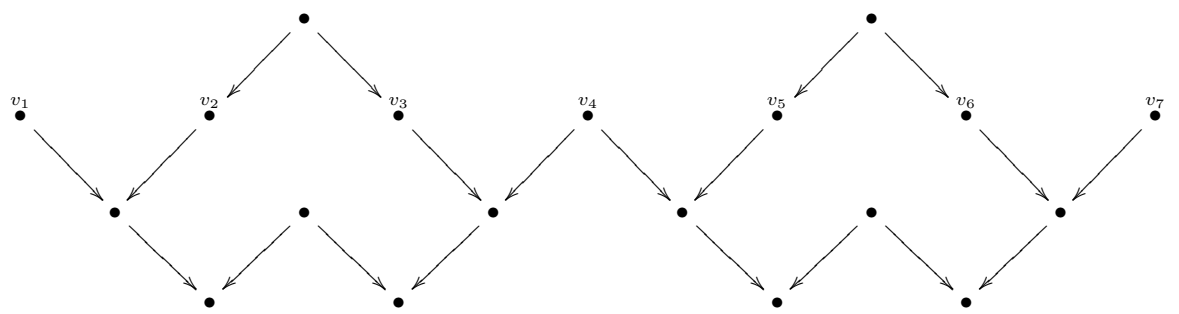

The kernel of $\Theta_{E}=s x+t y$ on $\Omega^{n} k \otimes k[s, t]$ is a submodule of a free $k[s, t]$-module generated by $v_{1}=x^{p-1} a_{1}, v_{2}=x^{p-2}\left(y a_{1}-x a_{2}\right), v_{3}=x^{p-3} y\left(y a_{1}-x a_{2}\right), \ldots, v_{p}=$ $y^{p-2}\left(y a_{1}-x a_{2}\right), v_{p+1}=y^{p-1} a_{2}-x^{p-1} a_{3}, \ldots, v_{\frac{n p}{2}}+1=y^{p-1} a_{n}$. Everything below this layer which is in $\operatorname{Ker} \Theta_{E}$ also $\operatorname{lies}$ in $\operatorname{Im} \Theta_{E}^{p-1}$. One verifies that the quotient

$\operatorname{Ker}\left\{\Theta_{E}: \Omega^{n} k \otimes k[s, t] \rightarrow \Omega^{n} k \otimes k[s, t]\right\} / \operatorname{Im}\left\{\Theta_{E}^{p-1}: \Omega^{n} k \otimes k[s, t] \rightarrow \Omega^{n} k \otimes k[s, t]\right\}$

is generated by

$$
s^{\frac{n p}{2}} v_{1}-s^{\frac{n p}{2}-1} t v_{2}+\cdots \pm t^{\frac{n p}{2}} v_{\frac{n p}{2}+1} .
$$

Arguing as in the proof of Proposition 6.13, we conclude that the corresponding locally free sheaf of rank 1 is $\mathcal{O}_{P^{1}}\left(-\frac{n p}{2}\right)$. The calculation for odd positive $n$ is similar. For negative values of $n$, one verifies the formula by again doing a similar calculation with dual modules.

Now let $r>2$, let $i: F \subset E$ be a subgroup scheme isomorphic to $\mathbb{G}_{a(1)}^{\times 2}$, and let $f: \mathbb{P}(F) \rightarrow \mathbb{P}(E)$ be the map induced by the embedding $i$. Since $\left(\Omega_{E}^{n} k\right) \downarrow_{F} \simeq$ $\Omega_{F}^{n} k \oplus$ proj, we conclude that $\mathcal{H}^{[1]}\left(\left(\Omega_{E}^{n} k\right) \downarrow_{F}\right) \simeq \mathcal{H}^{[1]}\left(\Omega_{F}^{n} k\right)$. Proposition 5.3 implies an isomorphism $f^{*}\left(\mathcal{H}^{[1]}\left(\Omega_{E}^{n} k\right)\right) \simeq \mathcal{H}^{[1]}\left(\left(\Omega_{E}^{n} k\right) \downarrow_{F}\right)$. Hence,

$$
f^{*}\left(\mathcal{H}^{[1]}\left(\Omega_{E}^{n} k\right)\right) \simeq \mathcal{H}^{[1]}\left(\Omega_{F}^{n} k\right) .
$$


The proposition now follows from the observation that

$$
f: \mathbb{P}(F) \simeq \mathbb{P}^{1} \longrightarrow \mathbb{P}(E) \simeq \mathbb{P}^{r-1}
$$

induces an isomorphism on Picard groups via $f^{*}$.

\section{REFERENCES}

[1] P. Balmer, D. Benson, J. Carlson, Gluing representations via idempotent modules and constructing endotrivial modules, J. of Pure and Applied Algebra 213, no. 2 (2009), pp. 173-193. MR2467395 (2009i:20016)

[2] G. Benkart, J.M. Osborn, Representations of rank one Lie algebras of characteristic p, Lie algebras and related topics (New Brunswick, N.J., 1981), pp. 1-37, Lecture Notes in Math., 933, Springer, Berlin-New York, 1982. MR675104 (84c:17004)

[3] D.J. Benson, Representations and cohomology, Volumes I and II, Cambridge University Press, 1991. MR.1110581 (92m:20005) MR:1156302 (93g:20099)

[4] D.J. Benson, Modules of constant Jordan type with one non-projective block, Algebr. Represent. Theory 13 (2010), no. 3, 315-318. MR2630123

[5] D. Benson and J. Pevtsova, Realization theorem for modules of constant Jordan type and vector bundles. To appear.

[6] J. Carlson, The varieties and the cohomology ring of a module, J. Algebra 85 (1983), 104-143. MR723070 (85a:20004)

[7] J. Carlson, E. Friedlander, Exact category of modules of constant Jordan type. Progr. Math., 269, Birkhauser Boston, Inc., Boston, MA, 2009. MR2641174

[8] J. Carlson, E. Friedlander, J. Pevtsova, Modules of constant Jordan type, Journal fúr die Reine und Angewandte Mathematik 614 (2008), 191-234. MR2376286 (2008j:20135)

[9] J. Carlson, E. Friedlander, A. Suslin, Modules over $\mathbb{Z} / p \times \mathbb{Z} / p$, to appear in Commentarrii Mathematici Helvetici.

[10] J. Carlson, L. Townsley, L. Valero-Elizondo, M. Zhang, Cohomology rings of finite groups, Kluwer, 2003. MR 2028960 (2004k:20110)

[11] M. Duflo, V. Serganova, On associated variety for Lie superalgebras.

[12] D. Eisenbud, Commutative algebra with a view towards algebraic geometry, Springer-Verlag, 1995. MR 1322960 (97a:13001)

[13] E. Friedlander, B. Parshall, Support varieties for restricted Lie algebras, Invent. Math. 86 (1986), 553-562. MR860682 (88f:17018)

[14] E. Friedlander, B. Parshall, Modular representation theory of Lie algebras, Amer. J Math. 110 (1988), 1055-1094. MR970120 (89j:17015)

[15] E. Friedlander, J. Pevtsova, Representation-theoretic support spaces for finite group schemes, Amer. J. Math. 127 (2005), 379-420. MR2130619 (2005k:14096)

[16] E. Friedlander, J. Pevtsova, Erratum: Representation-theoretic support spaces for finite group schemes, Amer. J. Math. 128 (2006), 1067-1068. MR2251594(2007d:14083)

[17] E. Friedlander, J. Pevtsova, П-supports for modules for finite group schemes, Duke. Math. J. 139 (2007), 317-368. MR2352134 (2008g:14081)

[18] E. Friedlander, J. Pevtsova, A. Suslin, Generic and maximal Jordan types, Invent. Math. 168 (2007), 485-522. MR.2299560(2008e:20072)

[19] E. Friedlander, A. Suslin, Cohomology of finite group scheme over a field, Invent. Math. 127 (1997), 235-253. MR1427618 (98h:14055a)

[20] R. Hartshorne, Algebraic geometry, Springer, 1977. MR0463157 (57:3116)

[21] J. Humphreys, Introduction to Lie algebras and representation theory, Springer, 1972. MR0323842(48:2197)

[22] J. Jantzen, Representations of algebraic groups, American Mathematical Society, 2003. MR2015057 (2004h:20061)

[23] G. McNinch, Abelian unipotent subgroups of reductive groups, J. of Pure and Applied Algebra, 167 (2002), 269-300. MR.1874545(2002i:20064)

[24] U. Oberst, H.-J. Schneider, Uber Untergruppen endlicher algebraischer Gruppen, Manuscripta Math. 8 (1973), 217-241. MR0347838 (50:339)

[25] A. Suslin, E. Friedlander, C. Bendel, Infinitesimal 1-parameter subgroups and cohomology, J. Amer. Math. Soc. 10 (1997), 693-728. MR1443546 (98h:14055b) 
[26] A. Suslin, E. Friedlander, C. Bendel, Support varieties for infinitesimal group schemes, J. Amer. Math. Soc. 10 (1997), 729-759. MR1443547 (98h:14055c)

[27] W. Waterhouse, Introduction to affine group schemes, Graduate Texts in Mathematics, 66 Springer-Verlag, New York-Berlin, 1979. MR547117 (82e:14003)

Department of Mathematics, University of Southern California, Los Angeles, CalIFORNIA 90089-2532

E-mail address: eric@math.northwestern.edu

E-mail address: ericmf@usc.edu

Department of Mathematics, University of Washington, Seattle, Washington 981954350

E-mail address: julia@math.washington.edu 